(c) 2006 International Press

Adv. Theor. Math. Phys. 10 (2006) 433-501

\title{
Analytic solution for tachyon condensation in open string field theory
}

\author{
Martin Schnabl \\ Department of Physics, Theory Division, CERN, CH-1211, \\ Geneva 23, Switzerland \\ martin.schnabl@cern.ch
}

\begin{abstract}
We propose a new basis in Witten's open string field theory, in which the star product simplifies considerably. For a convenient choice of gauge, the classical string field equation of motion yields straightforwardly an exact analytic solution that represents the nonperturbative tachyon vacuum. The solution is given in terms of Bernoulli numbers and the equation of motion can be viewed as novel Euler-Ramanujan-type identity. It turns out that the solution is the Euler-Maclaurin asymptotic expansion of a sum over wedge states with certain insertions. This new form is fully regular from the point of view of level truncation. By computing the energy difference between the perturbative and nonperturbative vacua, we prove analytically Sen's first conjecture.
\end{abstract}

e-print archive: http://lanl.arXiv.org/abs/hep-th/0511286 


\section{CONTEnTs}

1. Introduction 435

2. Star algebra $\quad 442$

2.1. The Fock space and the two-vertex 442

2.2. The three-vertex and the star product 445

2.3. Wedge states with insertions 448

2.4. Operator algebra in the $\tilde{z}$ coordinate 450

2.5. Star product in the $\mathcal{L}_{0}$-basis $\quad 454$

3. Ghost number zero toy model 458

3.1. "Tachyon" solutions 449

3.2. "Pure gauge" solutions 463

4. Ghost number one - the real thing 466

4.1. Proof of the equation of motion 469

4.2. Proof of Sen's first conjecture 470

4.3. Transforming to the Virasoro basis 473

4.4. Padé approximants and Borel summation 477

5. Conclusions and outlook 481

$\begin{array}{ll}\text { Acknowledgments } & 482\end{array}$

Appendix A. Comments on surface states 482

A.1. Representation in terms of Virasoro operators 482

A.2. Conservation laws $\quad 484$

$\begin{array}{lll}\text { Appendix B. } & \text { Bernoulli numbers } & 487\end{array}$

Appendix C. Proof of the sum-sliver cancellation 488 
Appendix D. Collection of useful formulas 490

$\begin{array}{lll}\text { D.1. } & \mathcal{B}_{0} \text {-gauge formulas } & 490\end{array}$

$\begin{array}{lll}\text { D.2. Some correlators } & 491\end{array}$

Appendix E Details for ghost number one equation of motion

492

E.1. Pure gauge solutions 496

Appendix F Coefficients of the tachyon condensate in the Virasoro basis 496

$\begin{array}{ll}\text { References } & 497\end{array}$

\section{Introduction}

Despite the beauty and simplicity of Witten's covariant field theory [1] for open bosonic string, only limited progress has been achieved over the years in practical applications $[2,3]$. The two main successes of the theory are computations of certain perturbative string amplitudes and understanding the phenomenon of tachyon condensation. It is fair to say nonetheless, that off-shell amplitudes in the Siegel gauge, the most popular covariant gauge, are rather unwieldy for practical purposes. To find explicitly even the simplest off-shell amplitudes, one has to resort to numerical methods. For the tachyon condensation, the situation is not much better. Putting aside the interesting vacuum string field theory proposal [4], most of the results so far were obtained by tedious numerical computations, following the seminal work of Sen and Zwiebach [5], using the method of level truncation [6].

The physics of tachyon condensation ${ }^{1}$ has made a major step forward when Ashoke Sen identified the open-string tachyon with a physical instability of the $D$-brane on which the open string ends. He made the following three conjectures [11,12]. First, he related the height of the tachyon potential at the true minimum to the tension of the $D$-brane on which the tachyon lives. Second, he predicted existence of lump solutions with correct tensions which describe lower dimensional $D$-branes popping out of the true vacuum. Finally, he conjectured that there are no physical excitations around the minimum and hence the cohomology of the BRST-like kinetic operator

\footnotetext{
${ }^{1}$ Some early papers on this issue include [7-10].
} 
there is empty. Sen's conjectures have been tested in variety of models, such as noncommutative field theory, $p$-adic string, boundary string field theory or vacuum string field theory. Within boundary string field theory, the first and second conjectures were proved in [13-15]. The third conjecture is true by construction in the vacuum string field theory and the first two conjectures in this model were proved in [16-18].

The most accurate, beautiful and complete formulation of open bosonic string field theory is Witten's cubic string field theory, but unfortunately due to the lack of exact analytic solutions, it allowed Sen's conjectures to be tested only numerically. The height of the tachyon potential has been tested with ever increasing accuracy in $[5,19,20]$. The second conjecture was tested in a number of interesting papers starting with [21-23] and the third one in [24-26]. For more references, we refer to the reviews $[2,3]$ and [27-30].

There was a large effort towards constructing analytic solutions. Various exact symmetries of the Siegel-gauge solution have been identified [31-33] and other were actively looked for [34]. Exact solutions were sought in the pure-gauge-like or partial-isometry form advocated in [35], but so far all such explicit solutions [36-38] contained the identity state of the string field algebra with some insertions and turned out to be singular. There was another class of papers [39-41], which attempted to find systematic analytic approximations to the exact solutions. Unfortunately, none of the above papers succeeded in proving Sen's conjectures perhaps with the exception of the third conjecture [42-44]. It is the goal of the present paper to provide the first nonsingular solution and prove Sen's first conjecture.

The reason why most computations are hard in string field theory is two-fold. First is that the three-string vertex itself $\left\langle V_{123}|| \psi_{1}\right\rangle \otimes\left|\psi_{2}\right\rangle \otimes\left|\psi_{3}\right\rangle$, which defines the product in the string field algebra $\left|\psi_{1}\right\rangle *\left|\psi_{2}\right\rangle=\left\langle\psi_{1}\right| \otimes$ $\left\langle\psi_{2}|| V_{123}\right\rangle$, is quite complicated, especially when expressed in the standard basis of $L_{0}$ eigenstates formed by matter and ghost oscillators. There is a basis in which the star product simplifies [45-49], but manifest background independence in the tachyon sector is lost and also conformal field theory techniques become rather cumbersome. The second reason that makes all the computations even harder is the choice of gauge fixing. Imposing the Siegel gauge $b_{0} \psi=0$ results in the propagator $b_{0} / L_{0}$. Now every nontrivial string field theory amplitude contains as part of its expression ${ }^{2}$

$$
\left|\psi_{1}\right\rangle * \frac{b_{0}}{L_{0}}\left(\left|\psi_{2}\right\rangle *\left|\psi_{3}\right\rangle\right)
$$

\footnotetext{
${ }^{2}$ For certain amplitudes, one does not need the full information about the star product (1.1). For example for the 4-point amplitude we need only the contraction $\left\langle\psi_{1}\left|*\left\langle\psi_{2}\left|\frac{b_{0}}{L_{0}}\right| \psi_{3}\right\rangle *\right| \psi_{4}\right\rangle=\left\langle I|| \psi_{1}\right\rangle *\left|\psi_{2}\right\rangle * \frac{b_{0}}{L_{0}}\left(\left|\psi_{3}\right\rangle *\left|\psi_{4}\right\rangle\right)$
} 
These building blocks of the string field theory Feynman diagrams have never been worked out explicitly, but it is clear that they can be extracted from general off-shell amplitudes that have been obtained in the past and that they are going to depend on Schwarz-Christoffel maps of polygons to the unit disk. Typically, the parameters specifying the map depend on propagator lengths (i.e., the Schwinger parameters) in a rather transcendental way [50-53].

The string world-sheet is usually parameterized by a complex strip coordinate $w=\sigma+i \tau, \sigma \in[0, \pi]$ or by $z=-\mathrm{e}^{-i w}=-\mathrm{e}^{-i \sigma+\tau}$, which takes values in the upper half-plane (UHP). As has been shown in [16], the gluing conditions entering the geometrical definition of the star product simplify if one uses another coordinate $\tilde{z}=\arctan z$, in which the UHP looks as a semiinfinite cylinder of circumference $\pi$. In fact, in this coordinate, we can write down simple closed form expression for arbitrary star products within the subalgebra generated by Fock space states. Elements of this subalgebra are finite sums of the so called wedge states with insertions $[54,55]$, which we shall write in the form

$$
U_{r}^{\dagger} U_{r} \tilde{\phi}_{1}\left(\tilde{x}_{1}\right) \tilde{\phi}_{2}\left(\tilde{x}_{2}\right) \cdots \tilde{\phi}_{n}\left(\tilde{x}_{n}\right)|0\rangle .
$$

By $\tilde{\phi}(\tilde{x})$, we denote a local operator $\phi(z)$ expressed in the $\tilde{z}$ coordinate, which in the special case of a primary field of dimension $h$ is given by

$$
\tilde{\phi}(\tilde{z})=\left(\frac{d z}{d \tilde{z}}\right)^{h} \phi(z)=(\cos \tilde{z})^{-2 h} \phi(\tan \tilde{z}) .
$$

The operator $U_{r}$ is a scaling operator in the $\tilde{z}$ coordinate, which can be written as $U_{r}=\left(\frac{2}{r}\right)^{\mathcal{L}_{0}}$, where

$$
\mathcal{L}_{0}=\oint \frac{d \tilde{z}}{2 \pi i} \tilde{z} T_{\tilde{z} \tilde{z}}(\tilde{z})
$$

is the zero mode of the worldsheet energy momentum tensor $T_{\tilde{z} \tilde{z}}$ in the $\tilde{z}$ coordinate. By a conformal transformation it can be expressed as

$$
\mathcal{L}_{0}=\oint \frac{d z}{2 \pi i}\left(1+z^{2}\right) \arctan z T_{z z}(z)=L_{0}+\sum_{k=1}^{\infty} \frac{2(-1)^{k+1}}{4 k^{2}-1} L_{2 k},
$$

where the $L_{n}$ 's are the ordinary Virasoro generators with zero central charge $c=0$ of the total (i.e., matter and ghost) conformal field theory. The operator $U_{r}^{\dagger}$ in (1.2) is hermitian conjugate of $U_{r}$, which in our particular case coincides with the BPZ conjugate. ${ }^{3}$

\footnotetext{
${ }^{3}$ Recall that the hermitian conjugate for a holomorphic field of dimension $h$ is $\phi_{n}^{\dagger}=\phi_{-n}$, whereas the BPZ conjugate is $\operatorname{bpz}\left(\phi_{n}\right)=(-1)^{n+h} \phi_{-n}$.
} 
At first glance, it might look surprising that we write (1.2) with the factor $U_{r}^{\dagger} U_{r}$ and not simply $U_{r}^{\dagger}$. After all, $U_{r}$ is just a scaling operator and its action on conformal fields of dimension $h$ is particularly simple

$$
U_{r} \tilde{\phi}(\tilde{z}) U_{r}^{-1}=\left(\frac{2}{r}\right)^{h} \tilde{\phi}\left(\frac{2}{r} \tilde{z}\right),
$$

and it also keeps the vacuum invariant $U_{r}|0\rangle=|0\rangle$. There are at least two reasons why we write (1.2) the way we write it. The first reason is that the star product of two such states takes a very simple form

$$
\begin{aligned}
& U_{r}^{\dagger} U_{r} \tilde{\phi}(\tilde{x})|0\rangle * U_{s}^{\dagger} U_{s} \tilde{\psi}(\tilde{y})|0\rangle \\
& \quad=U_{r+s-1}^{\dagger} U_{r+s-1} \tilde{\phi}\left(\tilde{x}+\frac{\pi}{4}(s-1)\right) \tilde{\psi}\left(\tilde{y}-\frac{\pi}{4}(r-1)\right)|0\rangle,
\end{aligned}
$$

where if there were more insertions, all insertions from the first string field would be shifted by $\pi(s-1) / 4$, whereas those from the second string field would move by $-\pi(r-1) / 4$. We shall give a detailed derivation of this formula in Section 2, although it follows easily from a similar expression in [55]. A nice feature of (1.7) is that it is valid for any local operator insertions, not necessarily primary fields. Second reason for writing our states in the form (1.2) will become clear later, when we discuss expansion of the string field in the $\mathcal{L}_{0}$ eigenstates.

A well-known special case of (1.2) are the wedge states $|r\rangle \equiv U_{r}^{\dagger}|0\rangle$ of Rastelli and Zwiebach [54]. They have no operator insertions (one can view it as an insertion of the operator identity) and by virtue of (1.7) they obey the simple algebra

$$
|r\rangle *|s\rangle=|r+s-1\rangle \text {. }
$$

This family of states is pretty rich by itself, since it contains the identity string field $|I\rangle=|1\rangle$ of the star algebra, the $S L(2, \mathbb{R})$ invariant vacuum $|0\rangle$ somewhat confusingly being the wedge state $|2\rangle$, multiple products of the vacua

$$
|n\rangle=\underbrace{|0\rangle *|0\rangle * \cdots *|0\rangle}_{(n-1) \text { times }}=U_{n}^{\dagger}|0\rangle=\left(\frac{2}{n}\right)^{\mathcal{L}_{0}^{\dagger}}|0\rangle,
$$

and finally it contains a peculiar projector $|\infty\rangle$ called the sliver.

Given the simplicity of the star product (1.7) in the $\tilde{z}$ coordinate, one may hope to be able to solve analytically the classical string field equation of motion $Q_{B} \Psi+\Psi * \Psi=0$ coming from Witten's action

$$
S=-\frac{1}{g_{o}^{2}}\left[\frac{1}{2}\left\langle\Psi, Q_{B} \Psi\right\rangle+\frac{1}{3}\langle\Psi, \Psi * \Psi\rangle\right] .
$$

Beautiful aspect of this action is its enormous gauge invariance $\delta \Psi=Q_{B} \Lambda+$ $\Psi * \Lambda-\Lambda * \Psi$ which, however, has to be fixed in one way or another unless 
one wants to deal with the full gauge orbit. The most popular choice for gauge fixing has been the Siegel gauge $b_{0} \Psi=0$. But alas, applying $b_{0} / L_{0}$ to the both sides of the equation of motion, one finds

$$
\Psi+\frac{b_{0}}{L_{0}}(\Psi * \Psi)=0,
$$

which cannot be solved easily within the states of the form (1.2), since application of the propagator $b_{0} / L_{0}=b_{0} \int_{t=0}^{\infty} \mathrm{e}^{-t L_{0}}$ leaves the family of wedge states with insertions. For this very reason, also the off-shell amplitudes in the Siegel gauge are doomed to be rather complicated.

We are thus led to look for other gauge choices. Most natural one, and as far as we can tell, the only one that works, is obtained by replacing the Siegel gauge $b_{0} \Psi=0$ with $\mathcal{B}_{0} \Psi=0$, where $\mathcal{B}_{0}$ is the zero mode of the $b$ ghost in the $\tilde{z}$ coordinate

$\mathcal{B}_{0}=\oint \frac{d \tilde{z}}{2 \pi i} \tilde{z} b_{\tilde{z} \tilde{z}}(\tilde{z})=\oint \frac{d z}{2 \pi i}\left(1+z^{2}\right) \arctan z b_{z z}(z)=b_{0}+\sum_{k=1}^{\infty} \frac{2(-1)^{k+1}}{4 k^{2}-1} b_{2 k}$.

Its anticommutator with the BRST charge $Q_{B}$ is $\left\{Q_{B}, \mathcal{B}_{0}\right\}=\mathcal{L}_{0}$ and hence, multiplying the equation of motion with $\mathcal{B}_{0} / \mathcal{L}_{0}$, which itself is part of the propagator, ${ }^{4}$ we can write analogously to (1.11) the 'projected' equation of motion as

$$
\Psi+\frac{\mathcal{B}_{0}}{\mathcal{L}_{0}}(\Psi * \Psi)=0 .
$$

It turns out that the operators $\mathcal{L}_{0}$ and $\mathcal{L}_{0}^{\dagger}$ obey a very simple algebra

$$
\left[\mathcal{L}_{0}, \mathcal{L}_{0}^{\dagger}\right]=\mathcal{L}_{0}+\mathcal{L}_{0}^{\dagger}
$$

and the algebra beautifully extends when generators $\mathcal{B}_{0}, \mathcal{B}_{0}^{\dagger}, B_{1}=b_{1}+b_{-1}$ and $K_{1}=L_{1}+L_{-1}$ are added to it. The Lie algebra (1.14) can be exponentiated and we find a Lie group with the property

$$
x^{\mathcal{L}_{0}} y^{\mathcal{L}_{0}^{\dagger}}=\left(\frac{y}{x+y-x y}\right)^{\mathcal{L}_{0}^{\dagger}}\left(\frac{x}{x+y-x y}\right)^{\mathcal{L}_{0}}
$$

which has a natural interpretation in terms of gluing of surfaces [55]. This relation allows for easy application of $\mathcal{B}_{0} / \mathcal{L}_{0}$ to a product of several Fock

\footnotetext{
${ }^{4}$ Actually, as we shall discuss elsewhere, the propagator in our gauge is equal to $\frac{\mathcal{B}_{0}}{\mathcal{L}_{0}} Q_{B} \frac{\mathcal{B}_{0}^{\dagger}}{\mathcal{L}_{0}^{\dagger}}$. Apparently, the presence of two Schwinger parameters for each propagator is the only disadvantage of our gauge. Note that the propagator in the Siegel gauge can be written in a similar form since $\frac{b_{0}}{L_{0}}=\frac{b_{0}}{L_{0}} Q_{B} \frac{b_{0}}{L_{0}}$.
} 
states of the form (1.2). For the wedge states, for example, we find

$$
\frac{\mathcal{B}_{0}}{\mathcal{L}_{0}}|r\rangle=-\mathcal{B}_{0}^{\dagger} \int_{2}^{r} \frac{d s}{s}|s\rangle,
$$

which apart of the $\mathcal{B}_{0}^{\dagger}$ factor is a superposition of states of the form (1.2). Enlarging our algebra of wedge states with insertions (1.2) by allowing for the explicit appearance of $\mathcal{B}_{0}^{\dagger}$, we find a simple sector of the star algebra closed not only under the star product, but also under the action of the BRST charge $Q_{B}$, the semi-propagator $\mathcal{B}_{0} / \mathcal{L}_{0}$ and many other operators.

The only method that has so far been used successfully for solving the string field theory equation of motion in the Siegel gauge is the level truncation [6]. Essentially one expands the string field in the eigenstates of the $L_{0}$ operator and truncates it to the first few levels, hoping that this presents a good approximation for the physical problem in question. This method has been very successful in finding the nonperturbative tachyon vacuum of the open strings $[5,19,20]$. As we have seen, the star algebra tremendously simplifies if one uses the $\tilde{z}$ coordinate. This leads us immediately to the possibility that the $\mathcal{L}_{0}$ level truncation might be the more natural one for the string field theory. It turns out that states of the form (1.2) have very simple expansion in terms of the $\mathcal{L}_{0}$ eigenstates. Unlike in the $L_{0}$ basis, where $U_{r}$ is rather complicated, in the $\mathcal{L}_{0}$ basis, the combination $U_{r}^{\dagger} U_{r}$ is equal to

$$
U_{r}^{\dagger} U_{r}=\sum_{n=0}^{\infty} \frac{1}{n !}\left(\frac{2-r}{2}\right)^{n} \widehat{\mathcal{L}}^{n}, \quad \widehat{\mathcal{L}} \equiv \mathcal{L}_{0}+\mathcal{L}_{0}^{\dagger}
$$

By (1.14), we see that the $n$-th term is an eigenstate (under the adjoint action) of $\mathcal{L}_{0}$ with eigenvalue $n$. Similarly, also the local operators in (1.2) can be naturally expanded in the basis of $\mathcal{L}_{0}$ eigenstates. For example, for the ghost field, we have

$$
\tilde{c}(\tilde{z})=\sum_{n=-\infty}^{\infty} \frac{\tilde{c}_{n}}{\tilde{z}^{n-1}},
$$

where $\tilde{c}_{n}$ are $\mathcal{L}_{0}$ eigenstates with eigenvalue $n$.

One rather unexpected feature arises when we combine the $\mathcal{B}_{0}$ gauge with the $\mathcal{L}_{0}$ level truncation in certain sector of the theory (formed by the $\tilde{c}_{n}$ modes, and $\widehat{\mathcal{L}}$ and $\widehat{\mathcal{B}} \equiv \mathcal{B}_{0}+\mathcal{B}_{0}^{\dagger}$ operators acting on the vacuum). The entire set of equations of motion for the individual components of $Q_{B} \Psi+\Psi * \Psi=$ 0 acquires such a simple structure that they can be solved exactly by a simple recursive procedure, level by level. The outcome of such a calculation 
is surprisingly so simple that a full all-levels form can be easily guessed to be

$$
\begin{aligned}
\Psi= & \sum_{n=0}^{\infty} \sum_{\substack{p=-1 \\
p \text { odd }}}^{\infty} \frac{\pi^{p}}{2^{n+2 p+1} n !}(-1)^{n} B_{n+p+1} \widehat{\mathcal{L}}^{n} \tilde{c}_{-p}|0\rangle \\
& +\sum_{n=0}^{\infty} \sum_{\substack{p, q=-1 \\
p+q \text { odd }}}^{\infty} \frac{\pi^{p+q}}{2^{n+2(p+q)+3} n !}(-1)^{n+q} B_{n+p+q+2} \widehat{\mathcal{B}} \widehat{\mathcal{L}}^{n} \tilde{c}_{-p} \tilde{c}_{-q}|0\rangle
\end{aligned}
$$

where $B_{n}$ are the Bernoulli numbers; see Appendix B for the definition and few basic properties.

Although a direct attempt to express solution (1.19) in the conventional $L_{0}$ basis gives rise to a divergent series, it turns out that (1.19) is the EulerMaclaurin asymptotic expansion of the following sum over wedge states with insertions

$$
\begin{aligned}
\Psi & =\lim _{N \rightarrow \infty}\left[\psi_{N}-\sum_{n=0}^{N} \partial_{n} \psi_{n}\right] \\
\psi_{n} & =\frac{2}{\pi^{2}} U_{n+2}^{\dagger} U_{n+2}\left[\widehat{\mathcal{B}} \tilde{c}\left(-\frac{\pi}{4} n\right) \tilde{c}\left(\frac{\pi}{4} n\right)+\frac{\pi}{2}\left(\tilde{c}\left(-\frac{\pi}{4} n\right)+\tilde{c}\left(\frac{\pi}{4} n\right)\right)\right]|0\rangle .
\end{aligned}
$$

As is well known, in most cases, the Euler-Maclaurin series are badly divergent (although they are often Borel summable as is the case here), so we should not be surprised by the divergence.

For the re-summed form (1.20), we prove that the solution is a true solution of the equation of motion, and we give a fully analytic proof of Sen's first conjecture. This new form is also suitable for the decomposition into the $L_{0}$ eigenstates. We find numerically that the coefficients are well behaved, higher level coefficients seem to decay quite rapidly and the solution resembles many features of the Siegel gauge solution $[5,19,20]$. This is in fact a rather pleasing feature of our gauge. Just as $\tan x \simeq x$ for small $x$, we have $\mathcal{B}_{0}=b_{0}+\frac{2}{3} b_{2}+\cdots$ and it seems that the dominant effect of the gauge fixing comes from the $b_{0}$ part. ${ }^{5}$ Also, truncating our exact solution to finite $L_{0}$ levels gives us a good approximation to the energy. A third way of arriving at the right energy is to start with the solution in the $\mathcal{L}_{0}$ basis and use Padé approximants. By this method, one confirms Sen's first conjecture with accuracy about $10^{-6}$ at level 18 .

\footnotetext{
${ }^{5}$ This proximity to the Siegel gauge distinguishes our $\mathcal{B}_{0}$ gauge from another interesting old proposal [56] which uses the star algebra derivative $B_{1}=b_{1}+b_{-1}$. The $B_{1}$ gauge shares some of the nice algebraic properties with the $\mathcal{B}_{0}$ gauge, but it seems to fail in describing the tachyon condensation.
} 
The paper is organized as follows. In Section 2, we will review and further develop properties of the star product using the $\tilde{z}$ coordinate. We will also prove a simple but powerful lemma, which will later allow direct construction of the tachyon vacuum. In Section 3, we will solve a simple toy model equation $\left(\mathcal{L}_{0}-1\right) \Phi+\Phi * \Phi=0$ whose solution will be given in terms of Bernoulli numbers. The equation of motion will become rather elegant and novel identity for the Bernoulli numbers, somewhat akin to the EulerRamanujan identities. This example will serve a useful lesson for the true string field theory with ghost number one string field in Section 4. Here, we shall describe how to find the solution and provide an alternative form useful for proving Sen's first conjecture, which we explicitly prove. Apart of the analytic proof, we provide two other rather distinct numerical confirmations, one using the Padé approximants and another one using ordinary level truncation. Some details are left for the appendices.

\section{Star algebra}

\subsection{The Fock space and the two-vertex}

The string field theory star algebra is an algebra built on the Hilbert space of the first quantized string. Postponing questions about its completeness, such a space must contain the Fock space, which we define here as the set of states created from the vacuum by the action of finitely many creation operators or equivalently by the insertion of local operators in the far past being represented by the puncture $P$ on the worldsheet, see figure 1 .

Traditionally, two coordinate systems have been used most often. The first, the more intuitive one, uses worldsheet time $\tau \in(-\infty, \infty)$ and coordinate $\sigma \in[0, \pi]$ which are often combined to form a new complex coordinate $w=\sigma+i \tau$ defined on a strip. Second coordinate system obtained by the map $z=-\mathrm{e}^{-i w}$ is the most practical one for the conformal field theory computations, since correlation functions on the UHP are easily found by the method of images.

For the purposes of the string field theory, a third coordinate system is the most useful one. It is obtained by the map $\tilde{z}=\arctan z$ which takes the UHP into the semi-infinite cylinder $C_{\pi}$ with circumference $\pi$. The conformal field theory in this coordinate remains easy. As in the case of the UHP, one can also employ the doubling trick to restrict our attention to a single holomorphic sector only. General $n$-point functions on $C_{\pi}$ can be readily 

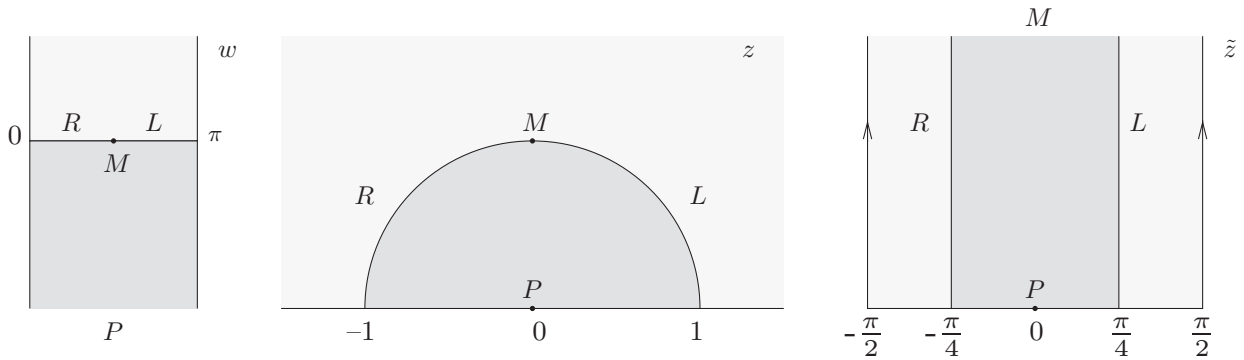

Figure 1: String worldsheet in three different coordinate systems related by $z=-\mathrm{e}^{-i w}$ and $\tilde{z}=\arctan z$. In the $\tilde{z}$ coordinate, the lines marked with an arrow are identified, so that the worldsheet forms semi-infinite cylinder $C_{\pi}$. Fock states are given by the insertion of local operators at the puncture $P$. Inserting operators also at $\tau=+\infty$, i.e., $z=\infty$ or $\tilde{z}=-\pi / 2=\pi / 2 \bmod \pi$ would correspond to taking the BPZ inner product. We have also marked the left and right (looking backwards in time) parts of the string at $\tau=0$ separated by the midpoint $M$.

found in terms of correlators on the UHP by conformal mapping ${ }^{6}$

$$
\left\langle\phi_{1}\left(\tilde{x}_{1}\right) \cdots \phi_{n}\left(\tilde{x}_{n}\right)\right\rangle_{C_{\pi}}=\left\langle\tilde{\phi}_{1}\left(\tilde{x}_{1}\right) \cdots \tilde{\phi}_{n}\left(\tilde{x}_{n}\right)\right\rangle_{\mathrm{UHP}}
$$

The fields $\tilde{\phi}_{i}\left(\tilde{x}_{i}\right)$ were defined in (1.3) as a coordinate change (i.e., a passive conformal transformation) of $\phi_{i}\left(x_{i}\right)$. Alternatively, they can be expressed as an active conformal transformation $\tilde{\phi}_{i}\left(\tilde{x}_{i}\right)=\tan \circ \phi_{i}\left(\tilde{x}_{i}\right)$, where, in general, $f \circ \mathcal{O}$ denotes an active conformal transformation of the operator $\mathcal{O}$. If, for example, $\mathcal{O}$ is a primary field $\phi(x)$ of dimension $h$, then $f \circ \phi(x)=$ $\left(f^{\prime}(x)\right)^{h} \phi(f(x))$. As we shall discuss below, the active conformal transformation can be represented by a similarity transformation on the string Hilbert space $f \circ \mathcal{O}=U_{f} \mathcal{O} U_{f}^{-1}$.

Consider, for example, the two and three-point functions. Let $\phi_{i}(z)$ be appropriately normalized holomorphic primary fields of dimension $h_{i}$. Then,

\footnotetext{
${ }^{6}$ In the modern language of string field theory [16,54], one uses a global coordinate $z$ defined on the UHP and local coordinates defined around punctures. In that approach, one never needs to discuss explicitly correlators anywhere else than in the UHP. It helps our intuition, however, to introduce at intermediate stages correlators of local operators on "real" cylinders, even though in practice they are evaluated by mapping them to the UHP. Care must be taken when translating formulas from one formalism to another. We thank Barton Zwiebach for a discussion that helped clarify this issue.
} 
the standard correlators in the UHP

$$
\begin{aligned}
\left\langle\phi_{i}(x) \phi_{j}(y)\right\rangle_{\mathrm{UHP}} & =\frac{\delta_{i j}}{(x-y)^{2 h_{i}}}, \\
\left\langle\phi_{i}(x) \phi_{j}(y) \phi_{k}(z)\right\rangle_{\mathrm{UHP}} & =\frac{C_{i j k}}{(x-y)^{h_{i}+h_{j}-h_{k}}(x-z)^{h_{i}+h_{k}-h_{j}}(y-z)^{h_{j}+h_{k}-h_{i}}}
\end{aligned}
$$

readily imply

$$
\begin{aligned}
& \left\langle\phi_{i}(\tilde{x}) \phi_{j}(\tilde{y})\right\rangle_{C_{\pi}}=\frac{\delta_{i j}}{\sin (\tilde{x}-\tilde{y})^{2 h_{i}}}, \\
& \left\langle\phi_{i}(\tilde{x}) \phi_{j}(\tilde{y}) \phi_{k}(\tilde{z})\right\rangle_{C_{\pi}} \\
& \quad=\frac{C_{i j k}}{\sin (\tilde{x}-\tilde{y})^{h_{i}+h_{j}-h_{k}} \sin (\tilde{x}-\tilde{z})^{h_{i}+h_{k}-h_{j}} \sin (\tilde{y}-\tilde{z})^{h_{j}+h_{k}-h_{i}}}
\end{aligned}
$$

on the semi-infinite cylinder $C_{\pi}$. The correlators are indeed well defined on $C_{\pi}$, as they are invariant under a shift of any of the coordinates by $\pi$, e.g., $x \rightarrow x+\pi$, provided that all dimensions $h_{i}$ are integer valued. Also note that the leading short distance behavior is the same for $C_{\pi}$ and UHP, as it should be.

As we have already mentioned, the Fock states are defined by insertions of local operators in the far past on the world-sheet

$$
|\phi\rangle=\phi(0)|0\rangle \text {. }
$$

But unless we are considering states corresponding to insertions of primary operators (on-shell states, for example), the states depend on the coordinate system used to insert the local operators. From the string field theory point of view, it is more natural to work with states

$$
|\tilde{\phi}\rangle=\tilde{\phi}(0)|0\rangle
$$

created from the vacuum by the insertion of $\phi(0)$ in the $\tilde{z}$ coordinate. By conformal transformation, this state can be expressed as

$$
\begin{aligned}
|\tilde{\phi}\rangle & =U_{\tan }|\phi\rangle \\
& =\exp \left[\frac{1}{3} L_{2}-\frac{1}{30} L_{4}+\frac{11}{1890} L_{6}-\frac{1}{1260} L_{8}-\frac{34}{467775} L_{10}+\cdots\right]|\phi\rangle,
\end{aligned}
$$

where $U_{\tan }$ is an operator which represents the action of conformal transformation $\tilde{z} \rightarrow z=\tan \tilde{z}$ and can be explicitly constructed following [54,58], see also [55]. Note that $U_{\tan }$ is the inverse of $U_{\arctan }$ which is used to define the sliver state [54].

In general, for any conformal map $f(z)$ holomorphic at $z=0$, one can construct the operator $U_{f}$ as an $\operatorname{exponential} \exp \left(\sum v_{n} L_{n}\right)$, where $n \geq 0$ and 
$v_{n}$ are Laurent coefficients of a vector field $v(z)=\sum v_{n} z^{n+1}$ related to the map $f(z)$ by the Julia equation $v(z) \partial_{z} f(z)=v(f(z))$. We should mention, however, that the vector field $v(z)$ often exists only as a formal power series, i.e., with zero radius of convergence. This is the case for $f(z)=\tan z$ and $f(z)=\arctan z$, (whose generating vector fields differ by an overall minus sign) as was shown in [55].

One of the key ingredients of the string field theory is the two-vertex, which is the familiar BPZ inner product of the conformal field theory, see figure 1 . It is defined as a map $\mathcal{H} \otimes \mathcal{H} \rightarrow \mathbb{R}$

$$
\left\langle\phi_{1}, \phi_{2}\right\rangle=\left\langle I \circ \phi_{1}(0) \phi_{2}(0)\right\rangle_{\mathrm{UHP}},
$$

where $I: z \rightarrow-1 / z$ is the inversion symmetry. For the states $\left|\tilde{\phi}_{i}\right\rangle$, the twovertex can be written as

$$
\left\langle\tilde{\phi}_{1}, \tilde{\phi}_{2}\right\rangle=\left\langle I \circ \tilde{\phi}_{1}(0) \tilde{\phi}_{2}(0)\right\rangle_{\mathrm{UHP}}=\left\langle\phi_{1}\left(\frac{\pi}{2}\right) \phi_{2}(0)\right\rangle_{C_{\pi}} .
$$

Note that in the $\tilde{z}$ coordinate, the inversion symmetry $I: z \rightarrow-1 / z$ becomes just a translation (i.e., a rotation) along the circumference $I: \tilde{z} \rightarrow \tilde{z} \pm \pi / 2$. The correlators (2.4) and (2.5) on $C_{\pi}$ are manifestly invariant under it.

\subsection{The three-vertex and the star product}

Unlike in the closed string field theory [59], in the open string field theory, there is a single-vertex which determines all the interactions. The threevertex is a map $\mathcal{H} \otimes \mathcal{H} \otimes \mathcal{H} \rightarrow \mathbb{R}$ and is defined as a correlator on a surface formed by gluing together three strips representing three open string worldsheets, see figure 2 .

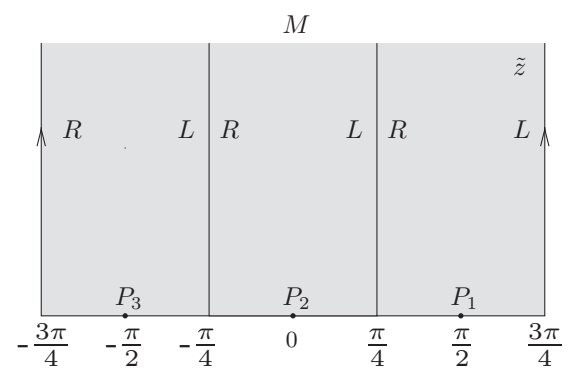

Figure 2: Worldsheet of disk topology (after adding the midpoint $M$ at infinity) glued together out of three semi-infinite strips. The lines marked with an arrow are identified. The three-vertex $\left\langle\phi_{1}, \phi_{2}, \phi_{3}\right\rangle$ is defined as a correlator of three local operators $\phi_{i}$ inserted in the punctures $P_{i}$. 
Traditionally $[54,57]$, for states $\left|\phi_{i}\right\rangle$ defined using the $z$ coordinate, the three-vertex has been written as

$$
\left\langle\phi_{1}, \phi_{2}, \phi_{3}\right\rangle=\left\langle f_{1} \circ \phi_{1}(0) f_{2} \circ \phi_{2}(0) f_{3} \circ \phi_{3}(0)\right\rangle_{\mathrm{UHP}}
$$

where $f_{n}(z)=\tan \left(\frac{(2-n) \pi}{3}+\frac{2}{3} \arctan z\right)$. For states defined using the $\tilde{z}$ coordinate, it can be expressed directly as

$$
\left\langle\tilde{\phi}_{1}, \tilde{\phi}_{2}, \tilde{\phi}_{3}\right\rangle=\left\langle\phi_{1}\left(\frac{\pi}{2}\right) \phi_{2}(0) \phi_{3}\left(-\frac{\pi}{2}\right)\right\rangle_{C_{3 \pi / 2}},
$$

without the need of any conformal map. Here, the correlator is taken on a semi-infinite cylinder $C_{3 \pi / 2}$ of circumference $3 \pi / 2$, see figure 2 .

The three-vertex allows us to introduce the star product $*: \mathcal{H} \otimes \mathcal{H} \rightarrow \mathcal{H}$. Given two states $\left|\phi_{1}\right\rangle$, and $\left|\phi_{2}\right\rangle$, the star product is defined by matching the three-vertex with an additional "test state" $|\chi\rangle$ to the two-vertex

$$
\left\langle\tilde{\chi}, \tilde{\phi}_{1}, \tilde{\phi}_{2}\right\rangle=\left\langle\tilde{\chi}, \tilde{\phi}_{1} * \tilde{\phi}_{2}\right\rangle, \quad \forall \chi .
$$

Graphically, the star product of two Fock states can be represented by the surface in figure 3. To find an explicit formula for the star product, it is useful to rewrite the left hand side of (2.13) as a correlator on a semi-infinite cylinder $C_{\pi}$ of circumference $\pi$

$$
\left\langle\tilde{\chi}, \tilde{\phi}_{1}, \tilde{\phi}_{2}\right\rangle=\left\langle s \circ \chi\left( \pm \frac{3 \pi}{4}\right) s \circ \phi_{1}\left(\frac{\pi}{4}\right) s \circ \phi_{2}\left(-\frac{\pi}{4}\right)\right\rangle_{C_{\pi}}
$$

using a simple conformal map $s: \tilde{z} \rightarrow \frac{2}{3} \tilde{z}$. Note that the scaling transformation $s$ is implemented by $U_{3} \equiv(2 / 3)^{\mathcal{L}}$, where $\mathcal{L}_{0}$ was introduced in (1.4) and (1.5). Thinking of $s \circ \phi_{1}\left(\frac{\pi}{4}\right) s \circ \phi_{2}\left(-\frac{\pi}{4}\right)$ in terms of its local operator product expansion around $\tilde{z}=0$, the right hand side of (2.14) has the form of the two-vertex (2.10). To see it more clearly, let us restrict to

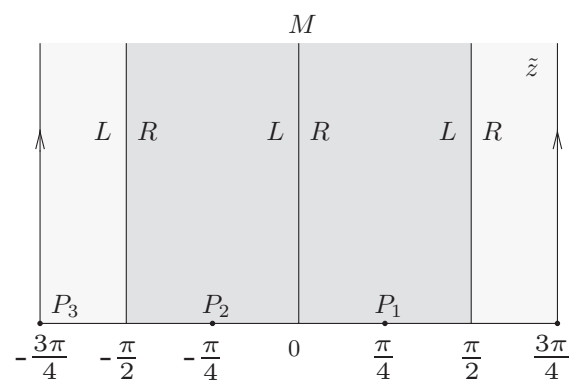

Figure 3: Star product of two states $\left|\tilde{\phi}_{1}\right\rangle *\left|\tilde{\phi}_{2}\right\rangle$ represented by local operator insertions at punctures $P_{1}$ and $P_{2}$. A local operator $\chi$ corresponding to the "test state" $|\tilde{\chi}\rangle$ can be inserted at the puncture $P_{3}$. The correlator is evaluated on a semi-infinite cylinder of circumference $3 \pi / 2$. 
the set of test states $\chi$ with definite scaling dimension $h$. Then, indeed $s \circ \chi( \pm 3 \pi / 4)=(2 / 3)^{h} \chi( \pm \pi / 2)$. Writing thus (2.14) as the two-vertex, the factor $(2 / 3)^{h}$ can be traded for an operator $U_{3}^{\dagger}$ acting on the second entry $s \circ \phi_{1}\left(\frac{\pi}{4}\right) s \circ \phi_{2}\left(-\frac{\pi}{4}\right)$, so that we have

$$
\left\langle\tilde{\chi}, \tilde{\phi}_{1}, \tilde{\phi}_{2}\right\rangle=\left\langle\tilde{\chi}, U_{3}^{\dagger}\left(s \circ \tilde{\phi}_{1}\left(\frac{\pi}{4}\right) s \circ \tilde{\phi}_{2}\left(-\frac{\pi}{4}\right)\right)\right\rangle
$$

and hence we find

$$
\tilde{\phi}_{1}(0)|0\rangle * \tilde{\phi}_{2}(0)|0\rangle=U_{3}^{\dagger} U_{3} \tilde{\phi}_{1}\left(\frac{\pi}{4}\right) \tilde{\phi}_{2}\left(-\frac{\pi}{4}\right)|0\rangle .
$$

When the local fields $\phi_{1,2}$ are, for example, primary fields of conformal dimensions $h_{1,2}$, we can use the fact that $U_{r}$ has a simple action (1.6) on them, and we can re-express (2.16) in the standard form

$$
\phi_{1}(0)|0\rangle * \phi_{2}(0)|0\rangle=\left(\frac{8}{9}\right)^{h_{1}+h_{2}} U_{3}^{\dagger} \phi_{1}\left(\tan \frac{\pi}{6}\right) \phi_{2}\left(-\tan \frac{\pi}{6}\right)|0\rangle .
$$

This formula agrees with few explicit examples given in [54] and generalized in [55]. It will be however formula (2.16), and its generalizations given in the next subsection, that will be most useful for the rest of the paper.

Let us now explain how to translate the expression (2.16) to the ordinary Virasoro basis based on the coordinate $z$. By Virasoro basis, we essentially mean the basis in the Verma module formed by the action of matter or total Virasoro generators on the highest weight states. In general, the operator $U_{r} \equiv(2 / r)^{\mathcal{L}_{0}}$ represents the scaling $\tilde{z} \rightarrow \frac{2}{r} \tilde{z}$, which in the $z$ coordinate becomes $z \rightarrow f_{r}(z)$, where

$$
f_{r}(z)=\tan \left(\frac{2}{r} \arctan z\right) .
$$

The operators $U_{r}$ can be written $\operatorname{as} \exp \left(\sum v_{n} L_{n}\right)$, by solving recursively the Julia equation $v(z) \partial_{z} f_{r}(z)=v\left(f_{r}(z)\right)$ following [54]. One finds

$$
\begin{aligned}
U_{r}= & \left(\frac{2}{r}\right)^{L_{0}} \exp \left[-\frac{r^{2}-4}{3 r^{2}} L_{2}+\frac{r^{4}-16}{30 r^{4}} L_{4}\right. \\
& -\frac{\left(r^{2}-4\right)\left(176+128 r^{2}+11 r^{4}\right)}{1890 r^{6}} L_{6} \\
& \left.+\frac{\left(r^{2}-4\right)\left(r^{2}+4\right)\left(16+32 r^{2}+r^{4}\right)}{1260 r^{8}} L_{8}+\cdots\right] .
\end{aligned}
$$

Using the composition rule $U_{f \circ g}=U_{f} U_{g}$, which reflects the fact that $U_{f}$ form a representation of the conformal group, one can arrive to a more 
convenient canonically ordered form ${ }^{7}$

$$
\begin{aligned}
U_{r}= & \left(\frac{2}{r}\right)^{L_{0}} \exp \left[-\frac{r^{2}-4}{3 r^{2}} L_{2}\right] \exp \left[\frac{r^{4}-16}{30 r^{4}} L_{4}\right] \\
& \times \exp \left[-\frac{16\left(r^{2}-4\right)\left(r^{2}-1\right)\left(r^{2}+5\right)}{945 r^{6}} L_{6}\right] \\
& \times \exp \left[\frac{\left(r^{2}-4\right)\left(109 r^{6}+436 r^{4}-944 r^{2}+1344\right)}{11340 r^{8}} L_{8}\right] \cdots,
\end{aligned}
$$

which is advantageous in level truncation computations. The least ordered, but most beautiful, form of $U_{r}$ is of course the one already mentioned

$U_{r}=\left(\frac{2}{r}\right)^{\mathcal{L}_{0}}=\exp \left[\log \left(\frac{2}{r}\right)\left(L_{0}+\frac{2}{3} L_{2}-\frac{2}{15} L_{4}+\frac{2}{35} L_{6}-\frac{2}{63} L_{8}+\cdots\right)\right]$.

\subsection{Wedge states with insertions}

So far, we have considered only a star product of two Fock states. Generalization to the multiple star product $\left|\tilde{\phi}_{1}\right\rangle *\left|\tilde{\phi}_{2}\right\rangle * \cdots *\left|\tilde{\phi}_{n}\right\rangle$, where $\left|\tilde{\phi}_{j}\right\rangle \equiv$ $\tilde{\phi}_{j}(0)|0\rangle$, is rather straightforward and is obtained by gluing together $n+1$ strips as in figure 4 . The analog of (2.16) is

$$
\begin{aligned}
\left|\tilde{\phi}_{1}\right\rangle *\left|\tilde{\phi}_{2}\right\rangle * \cdots *\left|\tilde{\phi}_{n}\right\rangle= & U_{n+1}^{\dagger} U_{n+1} \tilde{\phi}_{1}\left(\frac{(n-1) \pi}{4}\right) \tilde{\phi}_{2}\left(\frac{(n-3) \pi}{4}\right) \cdots \\
& \times \tilde{\phi}_{n}\left(-\frac{(n-1) \pi}{4}\right)|0\rangle
\end{aligned}
$$

In more generality, we could consider a family of states

$$
U_{r}^{\dagger} U_{r} \tilde{\phi}_{1}\left(\tilde{x}_{1}\right) \tilde{\phi}_{2}\left(\tilde{x}_{2}\right) \ldots \tilde{\phi}_{n}\left(\tilde{x}_{n}\right)|0\rangle
$$

for arbitrary real $r \geq 1$ and arbitrary insertion points $\tilde{x}_{i},\left|\operatorname{Re} \tilde{x}_{i}\right| \leq(r-1)$ $\pi / 4$. How do such states star multiply? States of the form (2.23) are represented by cylinders of circumference $r \pi / 2$ and punctures at points $\tilde{x}_{i}$ as in figure 4, regardless of whether they can be constructed by gluing Fock states or not. The star multiplication proceeds as for Fock states by simply gluing together the parts of the two or more cylinders with strips of length

\footnotetext{
${ }^{7}$ It is easy to write a simple recursive algorithm similar to the one of [54] to find out the coefficients in front of $L_{n}$ for almost arbitrarily high $n$. We provide more details in Appendix A.1.
} 


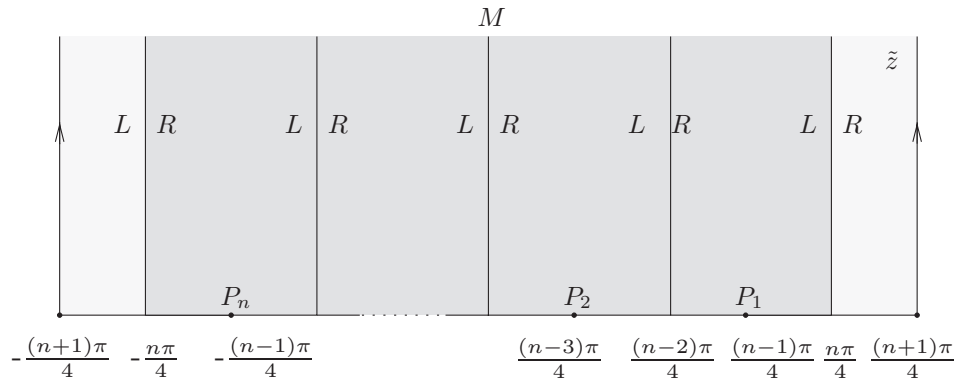

Figure 4: Multiple star product $\left|\tilde{\phi}_{1}\right\rangle *\left|\tilde{\phi}_{2}\right\rangle * \cdots *\left|\tilde{\phi}_{n}\right\rangle$, the so-called wedge state with insertions. Without insertions, it would be denoted as $|n+1\rangle$. The correlator is evaluated on a semi-infinite cylinder of circumference $(n+$ 1) $\pi / 2$.

$\pi / 2$ cut out (in light yellow in figure 4 ) and then gluing back one such strip to form a new bigger cylinder. ${ }^{8}$ Mathematically, we can write it as

$$
\begin{aligned}
& U_{r}^{\dagger} U_{r} \tilde{\phi}_{1}\left(\tilde{x}_{1}\right) \cdots \tilde{\phi}_{n}\left(\tilde{x}_{n}\right)|0\rangle * U_{s}^{\dagger} U_{s} \tilde{\psi}_{1}\left(\tilde{y}_{1}\right) \cdots \tilde{\psi}_{m}\left(\tilde{y}_{m}\right)|0\rangle \\
& =U_{t}^{\dagger} U_{t} \tilde{\phi}_{1}\left(\tilde{x}_{1}+\frac{\pi}{4}(s-1)\right) \cdots \tilde{\phi}_{n}\left(\tilde{x}_{n}+\frac{\pi}{4}(s-1)\right) \tilde{\psi}_{1}\left(\tilde{y}_{1}-\frac{\pi}{4}(r-1)\right) \cdots \\
& \quad \times \tilde{\psi}_{m}\left(\tilde{y}_{m}-\frac{\pi}{4}(r-1)\right)|0\rangle,
\end{aligned}
$$

where $t=r+s-1$. We leave it as an exercise to the reader to check the associativity.

Before we end this discussion, let us look in more detail on the simplest case with no insertions, i.e., when all operators $\phi_{i}$ are taken to be the identity operator. These are the original wedge states

$$
|r\rangle=U_{r}^{\dagger} U_{r}|0\rangle=U_{r}^{\dagger}|0\rangle
$$

introduced by Rastelli and Zwiebach in [54]. They obey a simple algebra

$$
|r\rangle *|s\rangle=|r+s-1\rangle,
$$

which is a special case of $(2.24)$. Note that the $S L(2, R)$ invariant vacuum $|0\rangle$ is the wedge state $|2\rangle$. The wedge state $|r\rangle$ with lowest allowed $r=1$ is the identity of the star algebra. For the limiting value $r \rightarrow \infty$, one finds a projector, the so-called sliver state, which has attracted much attention in the literature, especially in the context of the vacuum string field theory [4].

\footnotetext{
${ }^{8}$ Note that under star multiplication, the circumference can only grow or in a limiting case with $r=1$ can remain the same. Having $r<1$ would formally correspond to deleting a part of surface, it is hard to make sense of it in case there are some punctures and it is also ill behaved in level truncation [55].
} 


\subsection{Operator algebra in the $\tilde{z}$ coordinate}

To tackle such a complicated task such as solving the string field equations of motion, we found it very useful to use an operator formalism and to algebraize the problem. In fact our formula (2.24) was a first step in this program. Let us now take few steps further.

We have already noted that the wedge states can be naturally written in terms of the (hermitian or BPZ conjugate) of the scaling operator $U_{r}=$ $(2 / r)^{\mathcal{L}_{0}}$. The infinitesimal generator of the scaling is given by the zero mode $\mathcal{L}_{0}$ of the total energy momentum tensor $T_{\tilde{z} \tilde{z}}(\tilde{z})$ with zero central charge. Let us now look at other modes. We define

$$
\mathcal{L}_{n}=\oint \frac{d \tilde{z}}{2 \pi i} \tilde{z}^{n+1} T_{\tilde{z} \tilde{z}}(\tilde{z})=\oint \frac{d z}{2 \pi i}\left(1+z^{2}\right)(\arctan z)^{n+1} T_{z z}(z) .
$$

Note that there would be a central charge contribution in the last equation if $c$ were nonzero. The hermitian conjugate is then given by

$$
\mathcal{L}_{m}^{\dagger}=\oint \frac{d z}{2 \pi i}\left(1+z^{2}\right)(\operatorname{arccot} z)^{m+1} T_{z z}(z) .
$$

Both sets of operators obey standard Virasoro algebra with zero central charge.

$$
\begin{aligned}
& {\left[\mathcal{L}_{n}, \mathcal{L}_{m}\right]=(n-m) \mathcal{L}_{n+m},} \\
& {\left[\mathcal{L}_{n}^{\dagger}, \mathcal{L}_{m}^{\dagger}\right]=-(n-m) \mathcal{L}_{n+m}^{\dagger} .}
\end{aligned}
$$

What about the mixed commutators? It turns out that three operators $\mathcal{L}_{0}$, $\mathcal{L}_{0}^{\dagger}$ and $\mathcal{L}_{-1}=K_{1} \equiv L_{1}+L_{-1}$, which will be of particular importance, form an interesting closed algebra $[55,60]$

$$
\begin{aligned}
& {\left[\mathcal{L}_{0}, \mathcal{L}_{0}^{\dagger}\right]=\mathcal{L}_{0}+\mathcal{L}_{0}^{\dagger},} \\
& {\left[\mathcal{L}_{0}, K_{1}\right]=K_{1},} \\
& {\left[\mathcal{L}_{0}^{\dagger}, K_{1}\right]=-K_{1} .}
\end{aligned}
$$

There are three different ways of deriving it. The first, the most straightforward way, is to use the explicit form (1.5)

$$
\mathcal{L}_{0}=L_{0}+\sum_{k=1}^{\infty} \frac{2(-1)^{k+1}}{4 k^{2}-1} L_{2 k}
$$

and simply calculate the commutators as we did in [55]. The second, rather indirect way is to use the gluing theorem to argue [55] that

$$
U_{r} U_{s}^{\dagger}=U_{2+2 / r(s-2)}^{\dagger} U_{2+2 / s(r-2)} .
$$


Differentiating with respect to $r$ and $s$ and setting $r=s=2$, one recovers (2.31). The third method, which is also applicable for general modes $\mathcal{L}_{n}$, is to use standard contour arguments ${ }^{9}$ to find

$$
\begin{aligned}
{\left[\mathcal{L}_{n}, \mathcal{L}_{m}^{\dagger}\right]=} & \oint \frac{d z}{2 \pi i}\left(1+z^{2}\right)(\arctan z)^{n}(\operatorname{arccot} z)^{m} \\
& \times((m+1) \arctan z+(n+1) \operatorname{arccot} z) T(z)
\end{aligned}
$$

There is an important subtlety, however, in that the contours must pass precisely through the points $\pm i$; one can take the unit circle for example. The reason is that because of the cuts in $\arctan z$, the contour in (2.27) must cross the imaginary axis within the segment $[-i, i]$, whereas the contour in (2.28) must cross it outside this range. The choice of operator ordering is determined by the time ordering (i.e., the $|z|$-ordering in the radial quantization) in the path integral formalism, and therefore to make sense of the operator product $\mathcal{L}_{0} \mathcal{L}_{0}^{\dagger}$ in the path integral, the contour defining $\mathcal{L}_{0}^{\dagger}$ must lie inside the one defining $\mathcal{L}_{0}$. To satisfy these two conflicting requirements, the contours must pass through points $\pm i$, which are fortunately integrable singularities. This would not be the case for commutators $\left[\mathcal{L}_{r}, \mathcal{O}_{s}^{\dagger}\right]$, if $\mathcal{O}$ were an operator of dimension $h \leq 0$.

Some other nontrivial examples which can be obtained by this method are

$$
\begin{aligned}
& {\left[\mathcal{L}_{1}, \mathcal{L}_{1}^{\dagger}\right]=\frac{\pi^{2}}{6}\left(\mathcal{L}_{0}+\mathcal{L}_{0}^{\dagger}\right)-\frac{2}{3}\left(\mathcal{L}_{2}+\mathcal{L}_{2}^{\dagger}\right),} \\
& {\left[\mathcal{L}_{0}, \mathcal{L}_{1}^{\dagger}\right]=\frac{\pi^{2}}{4} \mathcal{L}_{-1}-\mathcal{L}_{1}}
\end{aligned}
$$

It is interesting to note that in general $\left[\mathcal{L}_{n}, \mathcal{L}_{m}^{\dagger}\right]$ are given as finite linear combinations of the generators $\mathcal{L}_{k}$ and $\mathcal{L}_{k}^{\dagger}$ as long as $n, m \geq-1$.

In terms of ordinary Virasoro operators, our new Virasoro operators are given explicitly by ${ }^{10}$

$$
\begin{aligned}
& \mathcal{L}_{2}=L_{2}-\frac{1}{15} L_{6}+\frac{64}{945} L_{8}+\cdots \\
& \mathcal{L}_{1}=L_{1}+\frac{1}{3} L_{3}-\frac{7}{45} L_{5}+\frac{29}{315} L_{7}+\cdots \\
& \mathcal{L}_{0}=L_{0}+\frac{2}{3} L_{2}-\frac{2}{15} L_{4}+\frac{2}{35} L_{6}+\cdots
\end{aligned}
$$

\footnotetext{
${ }^{9}$ I thank Ian Ellwood for suggesting the method.

${ }^{10} \mathrm{Had}$ we worked with nonzero central charge, the only modification would be an additional term $c / 6$ in $\mathcal{L}_{-2}$. Nevertheless, as shown in [55], some commutators such as $\left[\mathcal{L}_{0}, \mathcal{L}_{0}^{\dagger}\right]$ would become divergent.
} 


$$
\begin{aligned}
& \mathcal{L}_{-1}=L_{-1}+L_{1} \\
& \mathcal{L}_{-2}=L_{-2}+\frac{4}{3} L_{0}+\frac{11}{45} L_{2}-\frac{8}{189} L_{4} \cdots \\
& \mathcal{L}_{-3}=L_{-3}+\frac{5}{3} L_{-1}+\frac{3}{5} L_{1}-\frac{31}{945} L_{3} \cdots \\
& \mathcal{L}_{-4}=L_{-4}+2 L_{-2}+\frac{16}{15} L_{0}+\frac{62}{945} L_{2}-\frac{1}{225} L_{4} \cdots .
\end{aligned}
$$

Note that the operators $\mathcal{L}_{1}, \mathcal{L}_{0}, \mathcal{L}_{-1}, \mathcal{L}_{-2} \ldots$ are conservation laws for the sliver [61]. To see that, we note that $\mathcal{L}_{n}$ defined in (2.27) can be alternatively written as a conformal transformation of $L_{n}$

$$
\begin{aligned}
\mathcal{L}_{n} & =U_{\tan } L_{n} U_{\tan }^{-1}=\tan \circ L_{n}=\oint \frac{d z}{2 \pi i} z^{n+1} \tan \circ T_{z z}(z) \\
& =\oint \frac{d z}{2 \pi i} z^{n+1} \cos ^{-4} z T_{z z}(\tan z)=\oint \frac{d z}{2 \pi i}\left(1+z^{2}\right)(\arctan z)^{n+1} T_{z z}(z)
\end{aligned}
$$

and hence

$$
\langle\infty| \mathcal{L}_{-n}=\langle 0| U_{\arctan z} U_{\tan } L_{-n} U_{\tan }^{-1}=\langle 0| L_{-n} U_{\arctan z}=0
$$

for $n \geq-1$. We shall say more on the conservation laws for wedge states in Appendix A.2.

Attentive reader might have noticed from (2.31) that the combination $\mathcal{L}_{0}+\mathcal{L}_{0}^{\dagger}$ commutes with $K_{1}$. In fact there is a deeper reason for that. Note that from (2.27) and (2.28)

$$
\begin{aligned}
\mathcal{L}_{0}+\mathcal{L}_{0}^{\dagger} & =\oint \frac{d z}{2 \pi i}\left(1+z^{2}\right)(\arctan z+\operatorname{arccot} z) T(z) \\
& =\frac{\pi}{2} \oint \frac{d z}{2 \pi i}\left(1+z^{2}\right) \varepsilon(\operatorname{Re} z) T(z)
\end{aligned}
$$

where $\varepsilon(x)$ is the step function equal to \pm 1 for positive or negative values, respectively. (We also abbreviate $T_{z z}(z)$ to $T(z)$.) In order to be able to write expression (2.41) for both terms using a single contour integral, we have used the unit circle in both (2.27) and (2.28). Splitting the integration contour into two halves in (2.42), one in the $\operatorname{Re} z>0$ half-plane and the other in $\operatorname{Re} z<0$, we observe that these two semi-circle contour integrals are in fact the definition of $K_{1}^{L}$ and $K_{1}^{R}$, respectively. We thus find

$$
\mathcal{L}_{0}+\mathcal{L}_{0}^{\dagger}=\frac{\pi}{2}\left(K_{1}^{L}-K_{1}^{R}\right)
$$


and since $K_{1}^{L}+K_{1}^{R}=K_{1}$, we also have

$$
\begin{aligned}
K_{1}^{L} & =\frac{1}{2} K_{1}+\frac{1}{\pi}\left(\mathcal{L}_{0}+\mathcal{L}_{0}^{\dagger}\right) \\
K_{1}^{R} & =\frac{1}{2} K_{1}-\frac{1}{\pi}\left(\mathcal{L}_{0}+\mathcal{L}_{0}^{\dagger}\right)
\end{aligned}
$$

Now, we see that the relation $\left[\mathcal{L}_{0}+\mathcal{L}_{0}^{\dagger}, K_{1}\right]=0$ is responsible for $\left[K_{1}^{L}, K_{1}^{R}\right]$ $=0$. Here we are quite lucky, since such commutators between the left and right string operators are often anomalous.

The operators $K_{1}^{L}, K_{1}^{R}$ and $K_{1}$ also have rather simple properties with regard to the star product

$$
\begin{aligned}
K_{1}^{L}\left(\phi_{1} * \phi_{2}\right) & =\left(K_{1}^{L} \phi_{1}\right) * \phi_{2}, \\
K_{1}^{R}\left(\phi_{1} * \phi_{2}\right) & =\phi_{1} *\left(K_{1}^{R} \phi_{2}\right), \\
K_{1}\left(\phi_{1} * \phi_{2}\right) & =\left(K_{1} \phi_{1}\right) * \phi_{2}+\phi_{1} *\left(K_{1} \phi_{2}\right) .
\end{aligned}
$$

The first two relations reflect the geometry of the Witten vertex, in that the left part of the first string becomes the left part of the product and the right part of the right string becomes the right part of the star product. The last relation is the well-known fact that $K_{1}$ is a derivation of the star product. Sometimes an analogous relation might also be useful

$$
D\left(\phi_{1} * \phi_{2}\right)=\left(D \phi_{1}\right) * \phi_{2}+\phi_{1} *\left(D \phi_{2}\right),
$$

where $D=\mathcal{L}_{0}-\mathcal{L}_{0}^{\dagger}$ is another star algebra derivative. The operators $K_{1}^{L}$ and $K_{1}^{R}$ play a further role in that their operator action generates star multiplication by the family of wedge states for the full Hilbert space. Explicitly, as follows readily from the results in [55], we find

$$
\begin{aligned}
& |n\rangle *|\psi\rangle=\mathrm{e}^{-(n-1)(\pi / 2) K_{1}^{L}}|\psi\rangle, \\
& |\psi\rangle *|n\rangle=\mathrm{e}^{(n-1)(\pi / 2) K_{1}^{R}}|\psi\rangle .
\end{aligned}
$$

One can thus alternatively write the wedge states as ${ }^{11}$

$$
\begin{aligned}
|n\rangle & =\mathrm{e}^{-(n-2)(\pi / 2) K_{1}^{L}}|0\rangle=\mathrm{e}^{(n-2)(\pi / 2) K_{1}^{R}}|0\rangle=\mathrm{e}^{-(n-2)(\pi / 4)\left(K_{1}^{L}-K_{1}^{R}\right)}|0\rangle \\
& =\mathrm{e}^{-(n-2) / 2\left(\mathcal{L}_{0}+\mathcal{L}_{0}^{\dagger}\right)}|0\rangle .
\end{aligned}
$$

\footnotetext{
${ }^{11}$ One could also write the wedge states as $|n\rangle=\mathrm{e}^{-(n-1)(\pi / 2) K_{1}^{L}}|I\rangle$, which is reminiscent of the formal considerations in [36].
} 
Number of interesting relations can be obtained by exponentiating the Lie algebra (2.31). The most important ones are

$$
\begin{aligned}
U_{r} U_{s} & =U_{r s / 2}, \\
U_{r} U_{s}^{\dagger} & =U_{2+2 / r(s-2)}^{\dagger} U_{2+2 / s(r-2)}, \\
U_{r} \mathrm{e}^{\alpha X} & =\mathrm{e}^{(2 \alpha / r) X} U_{r}, \quad \text { valid for } X=K_{1}, K_{1}^{L, R}, \mathcal{L}_{0}+\mathcal{L}_{0}^{\dagger}, \\
\mathrm{e}^{\beta\left(\mathcal{L}_{0}+\mathcal{L}_{0}^{\dagger}\right)} & =U_{2-2 \beta}^{\dagger} U_{2-2 \beta} .
\end{aligned}
$$

The first two were derived in [55], and the latter two can be obtained by similar methods. Let us illustrate such a derivation on (2.56) which plays a central role in this paper. Let us denote $f(x)=x^{\mathcal{L}_{0}^{\dagger}} x^{\mathcal{L}_{0}}$. Clearly $f(1)=1$. The derivative $f^{\prime}(x)$ can be easily computed with the help of (2.57) given below

$$
f^{\prime}(x)=\frac{1}{x} x^{\mathcal{L}_{0}^{\dagger}}\left(\mathcal{L}_{0}^{\dagger}+\mathcal{L}_{0}\right) x^{\mathcal{L}_{0}}=\frac{1}{x^{2}} f(x)\left(\mathcal{L}_{0}^{\dagger}+\mathcal{L}_{0}\right)=\frac{1}{x^{2}}\left(\mathcal{L}_{0}^{\dagger}+\mathcal{L}_{0}\right) f(x) .
$$

Integrating this as a differential equation, we find $f(x)=\exp \left[(1-1 / x)\left(\mathcal{L}_{0}^{\dagger}+\right.\right.$ $\left.\mathcal{L}_{0}\right)$ and (2.56) readily follows. ${ }^{12}$ Other useful identities are

$$
\begin{aligned}
U_{r} \mathcal{L}_{0}^{\dagger} U_{r}^{-1} & =\frac{2-r}{r} \mathcal{L}_{0}+\frac{2}{r} \mathcal{L}_{0}^{\dagger}, \\
U_{r}^{\dagger-1} \mathcal{L}_{0} U_{r}^{\dagger} & =\frac{2}{r} \mathcal{L}_{0}+\frac{2-r}{r} \mathcal{L}_{0}^{\dagger}, \\
U_{r}^{-1} \mathcal{L}_{0}^{\dagger} U_{r} & =\frac{r-2}{2} \mathcal{L}_{0}+\frac{r}{2} \mathcal{L}_{0}^{\dagger}, \\
U_{r}^{\dagger} \mathcal{L}_{0} U_{r}^{\dagger-1} & =\frac{r}{2} \mathcal{L}_{0}+\frac{r-2}{2} \mathcal{L}_{0}^{\dagger} .
\end{aligned}
$$

Finally, for completeness, we remind the reader that on a primary field $\tilde{\phi}$ of dimension $h$, the exponentiated generators $\mathcal{L}_{0}$ and $K_{1}$ act as scaling and translation

$$
\begin{aligned}
\lambda^{\mathcal{L}_{0}} \tilde{\phi}(\tilde{z}) \lambda^{-\mathcal{L}_{0}} & =\lambda^{h} \tilde{\phi}(\lambda \tilde{z}), \\
\mathrm{e}^{\alpha K_{1}} \tilde{\phi}(\tilde{z}) \mathrm{e}^{-\alpha K_{1}} & =\tilde{\phi}(\tilde{z}+\alpha) .
\end{aligned}
$$

\subsection{Star product in the $\mathcal{L}_{0}$-basis}

It turns out that the most general string field algebra elements (2.23) we have considered so far can be very naturally expressed in the basis of $\mathcal{L}_{0}$

\footnotetext{
${ }^{12}$ Barton Zwiebach has suggested alternative derivation based on embedding the twodimensional algebra $\left[\mathcal{L}_{0}, \mathcal{L}_{0}^{\dagger}\right]=\mathcal{L}_{0}+\mathcal{L}_{0}^{\dagger}$ inside $g l(2)$ and using its explicit representation in terms of two-dimensional matrices.
} 
eigenstates. To start with, consider first pure wedge states with no insertions. They can be written using (2.56) as

$$
|r\rangle=U_{r}^{\dagger} U_{r}|0\rangle=\mathrm{e}^{(2-r) / 2\left(\mathcal{L}_{0}+\mathcal{L}_{0}^{\dagger}\right)}|0\rangle=\sum_{n=0}^{\infty} \frac{1}{n !}\left(\frac{2-r}{2}\right)^{n}\left(\mathcal{L}_{0}+\mathcal{L}_{0}^{\dagger}\right)^{n}|0\rangle .
$$

Note that by $(2.31)$ the states $\left(\mathcal{L}_{0}+\mathcal{L}_{0}^{\dagger}\right)^{n}|0\rangle$ are eigenstates of $\mathcal{L}_{0}$ with eigenvalue $n$. Although these states are far from being normal ordered, they are quite convenient. Almost normal ordered expression (normal ordered up to some $L_{0}$ 's hidden inside $\mathcal{L}_{0}^{\dagger}$ ) can be written as

$$
\begin{aligned}
\left(\mathcal{L}_{0}+\mathcal{L}_{0}^{\dagger}\right)^{n}|0\rangle & =\left(n-1+\mathcal{L}_{0}^{\dagger}\right)\left(n-2+\mathcal{L}_{0}^{\dagger}\right) \cdots\left(1+\mathcal{L}_{0}^{\dagger}\right) \mathcal{L}_{0}^{\dagger}|0\rangle=\frac{\Gamma\left(\mathcal{L}_{0}^{\dagger}+n\right)}{\Gamma\left(\mathcal{L}_{0}^{\dagger}\right)}|0\rangle \\
& =\sum_{k=1}^{n}(-1)^{n-k} S_{n}^{(k)}\left(\mathcal{L}_{0}^{\dagger}\right)^{k}|0\rangle
\end{aligned}
$$

where $S_{n}^{(k)}$ are the (signed) Stirling numbers of the first kind. They are defined in such a way that $(-1)^{n-k} S_{n}^{(k)}$ is the number of permutations of $n$ symbols which have precisely $k$ cycles. This expression might be useful for deriving various startling mathematical identities, but for our purposes, it will be the form $\left(\mathcal{L}_{0}+\mathcal{L}_{0}^{\dagger}\right)^{n}|0\rangle$ which will prove to be most useful.

There is a second kind of $\mathcal{L}_{0}$ eigenstates, which are perhaps more obvious, which are obtained simply by conformal transformation (2.8) of the $L_{0}$ eigenstates. As an example, consider modes of the $\tilde{c}$ ghost

$$
\tilde{c}(\tilde{z})=\sum_{n=-\infty}^{\infty} \frac{\tilde{c}_{n}}{\tilde{z}^{n-1}}
$$

given by

$$
\tilde{c}_{n}=\tan \circ c_{n}=\sum_{m=n}^{\infty} c_{m} \oint \frac{d \tilde{z}}{2 \pi i} \tilde{z}^{n-2} \cos ^{2} \tilde{z}(\tan \tilde{z})^{-m+1},
$$

since $\tilde{c}(\tilde{z})=\tan \circ c(\tilde{z})=\cos ^{2} \tilde{z} c(\tan \tilde{z})$. Equivalently, using the more conventional passive viewpoint, these modes can be expressed as

$$
\tilde{c}_{n}=\oint \frac{d \tilde{z}}{2 \pi i} \tilde{z}^{n-2} \tilde{c}(\tilde{z})=\sum_{m=n}^{\infty} c_{m} \oint \frac{d z}{2 \pi i} \frac{1}{\left(1+z^{2}\right)^{2}}(\arctan z)^{n-2} z^{-m+1} .
$$


First few $\mathcal{L}_{0}$ eigenstates are explicitly given by

$$
\begin{aligned}
\tilde{c}_{1}|0\rangle & =c_{1}|0\rangle \\
\tilde{c}_{0}|0\rangle & =c_{0}|0\rangle \\
\tilde{c}_{-1}|0\rangle & =\left(c_{-1}-c_{1}\right)|0\rangle \\
\tilde{c}_{-2}|0\rangle & =\left(c_{-2}-\frac{2}{3} c_{0}\right)|0\rangle \\
\tilde{c}_{-3}|0\rangle & =\left(c_{-3}-\frac{1}{3} c_{-1}+\frac{1}{3} c_{1}\right)|0\rangle .
\end{aligned}
$$

More complicated examples are given by products of several $\tilde{\phi}_{n}$ modes of any number of primary fields. Just to give an example of a case where there are contractions between two mode operators, we use (2.38) to write a weight 5 $\mathcal{L}_{0}$ eigenstate

$$
\mathcal{L}_{-3} \mathcal{L}_{-2}|0\rangle=\left(L_{-3} L_{-2}+\frac{5}{3} L_{-3}\right)|0\rangle
$$

We have seen that there are basically two types of $\mathcal{L}_{0}$ eigenstates. Ones which use an $n$-th power of $\mathcal{L}_{0}+\mathcal{L}_{0}^{\dagger}$ (or a factor of $\mathcal{B}_{0}+\mathcal{B}_{0}^{\dagger}$ ) and ones which use modes of primary operators $\tilde{\phi}_{n}$. The former ones contain infinite sum of terms in the ordinary $L_{0}$ basis, whereas the second ones only finite number of them. Looking at (2.23), we see that we really should combine and use these two kinds of states together. One might be worried about overcounting if we include both kinds of states, but note that, for instance, the state $\mathcal{L}_{0}^{\dagger}|0\rangle$ with $\mathcal{L}_{0}$ eigenvalue equal to one is truly impossible to write as a linear combination of states like $\tilde{\phi}_{n}|0\rangle$. In fact, the only viable candidate $\mathcal{L}_{-1}|0\rangle$ is identically equal to zero.

The star product rules for the above states of the $\mathcal{L}_{0}$ basis can be readily worked out using (2.24). This leads to the following trivial but powerful lemma which belongs to the main results of the paper:

Lemma. Let $\psi_{1}$ and $\psi_{2}$ be two eigenstates of $\mathcal{L}_{0}$ with eigenvalues $h_{1}$ and $h_{2}$, respectively. Let us further assume that they are linear combinations of states of the form (2.23) with the only operator insertions allowed being $\mathcal{B}_{0}^{\dagger}$, arbitrary power of $\mathcal{L}_{0}^{\dagger}$ and any number of the $\tilde{c}$ ghosts. Then, the star product $\psi_{1} * \psi_{2}$ is an infinite linear combination of $\mathcal{L}_{0}$ eigenstates with eigenvalues $h \geq h_{1}+h_{2}$. 
Proof. Let us write a basis of states with a definite $\mathcal{L}_{0}$ eigenvalue $h$ in the form

$$
\begin{gathered}
\left(\mathcal{L}_{0}+\mathcal{L}_{0}^{\dagger}\right)^{n} \tilde{c}_{-p_{1}} \tilde{c}_{-p_{2}} \cdots \tilde{c}_{-p_{k}}|0\rangle \\
\left(\mathcal{B}_{0}+\mathcal{B}_{0}^{\dagger}\right)\left(\mathcal{L}_{0}+\mathcal{L}_{0}^{\dagger}\right)^{m} \tilde{c}_{-q_{1}} \tilde{c}_{-q_{2}} \cdots \tilde{c}_{-q_{l}}|0\rangle,
\end{gathered}
$$

where $h=n+p_{1}+\cdots+p_{k}=1+m+q_{1}+\cdots+q_{l}$. The first basis element (2.67) can be rewritten up to a numerical factor as

$$
\left.\frac{d^{n+\left(p_{1}+1\right)+\cdots+\left(p_{k}+1\right)}}{d r^{n} d \tilde{x}_{1}^{\left(p_{1}+1\right)} \cdots d \tilde{x}_{k}^{p_{k}+1}} U_{r}^{\dagger} U_{r} \tilde{c}\left(\tilde{x}_{1}\right) \cdots \tilde{c}\left(\tilde{x}_{k}\right)|0\rangle\right|_{\substack{r=2 \\ \tilde{x}_{i}=0}} .
$$

Multiplying two states of this form using the formula (1.7) or (2.24)

$$
\begin{aligned}
& U_{r}^{\dagger} U_{r} \tilde{\phi}_{1}(\tilde{x})|0\rangle * U_{s}^{\dagger} U_{s} \tilde{\phi}_{2}(\tilde{y})|0\rangle \\
& \quad=U_{r+s-1}^{\dagger} U_{r+s-1} \tilde{\phi}_{1}\left(\tilde{x}+\frac{\pi}{4}(s-1)\right) \tilde{\phi}_{2}\left(\tilde{y}-\frac{\pi}{4}(r-1)\right)|0\rangle,
\end{aligned}
$$

we see that the total number of derivatives acting on the right hand side will be equal to the sum of the number of derivatives acting on the two factors on the left hand side. Some of the derivatives on the right hand side can act on both $U_{t}^{\dagger} U_{t}$ and $\tilde{c}$ ghosts, but regardless of where they act they always increase the $\mathcal{L}_{0}$ eigenvalue by 1 . Since setting $r=s=2$ at the end leaves us with $U_{3}^{\dagger} U_{3}$ apart of powers of $\mathcal{L}_{0}+\mathcal{L}_{0}^{\dagger}$ and modes of the $\tilde{c}$ ghosts, we have proven only $h \geq h_{1}+h_{2}$ and not the equality.

For the states (2.68), we may use the identities (see Appendix D.1)

$$
\begin{aligned}
\left(\left(\mathcal{B}_{0}+\mathcal{B}_{0}^{\dagger}\right) \phi_{1}\right) * \phi_{2}= & \left(\mathcal{B}_{0}+\mathcal{B}_{0}^{\dagger}\right)\left(\phi_{1} * \phi_{2}\right)+(-1)^{\operatorname{gh}\left(\phi_{1}\right)} \frac{\pi}{2} \phi_{1} * B_{1} \phi_{2}, \\
\phi_{1} *\left(\left(\mathcal{B}_{0}+\mathcal{B}_{0}^{\dagger}\right) \phi_{2}\right)= & (-1)^{\operatorname{gh}\left(\phi_{1}\right)}\left(\mathcal{B}_{0}+\mathcal{B}_{0}^{\dagger}\right)\left(\phi_{1} * \phi_{2}\right) \\
& -(-1)^{\operatorname{gh}\left(\phi_{1}\right)} \frac{\pi}{2}\left(B_{1} \phi_{1}\right) * \phi_{2}, \\
\left(\left(\mathcal{B}_{0}+\mathcal{B}_{0}^{\dagger}\right) \phi_{1}\right) *\left(\left(\mathcal{B}_{0}+\mathcal{B}_{0}^{\dagger}\right) \phi_{2}\right)= & -(-1)^{\operatorname{gh}\left(\phi_{1}\right)} \frac{\pi}{2}\left(\mathcal{B}_{0}+\mathcal{B}_{0}^{\dagger}\right) B_{1}\left(\phi_{1} * \phi_{2}\right) \\
& +\left(\frac{\pi}{2}\right)^{2}\left(B_{1} \phi_{1}\right) *\left(B_{1} \phi_{2}\right),
\end{aligned}
$$

and thanks to the fact that $B_{1}$ and $\left(\mathcal{B}_{0}+\mathcal{B}_{0}^{\dagger}\right)$ raise the $\mathcal{L}_{0}$ eigenvalue by one, we can reduce this case to the previous one.

Let us note that the lemma in its simplest form holds only for the assumed subsector of the string field theory, which fortunately is big enough for the goals of the present paper. As soon as one starts to introduce other operator insertions such as matter operators $\partial X$, $\mathrm{e}^{i k X}$, etc., operator contractions seem to spoil the nice property that $h \geq h_{1}+h_{2}$. It would be nice to find 
a way out in order to be able to look efficiently for space-time-dependent solutions. For the case of Wilson line marginal deformations generated by $i \partial X$, one possibility might be to replace in the above lemma the operator $\mathcal{L}_{0}$ with $\mathcal{L}_{0}+N$, where $N$ is an $\alpha^{\prime}$ counting operator. This could work, since each contraction of $i \partial X^{\prime}$ 's is accompanied by an explicit factor of $\alpha^{\prime}$.

\section{Ghost number zero toy model}

It has been suggested [34] that since $L_{0}$ has eigenvalue -1 on $c_{1}|0\rangle$, solving an equation $\left(L_{0}-1\right)|\Phi\rangle+|\Phi\rangle *|\Phi\rangle=0$ for ghost number zero field $\Phi$ could teach us something about the true ghost number one solution. Indeed it was found that some of the coefficients of the tachyon solution in the matter sector of the ghost number one theory were strikingly close to the corresponding coefficients in the ghost number zero solutions. Although the precise relationship has never been discovered, and if it exists it is very likely not a simple one, we shall start with an analogous equation

$$
\left(\mathcal{L}_{0}-1\right) \Phi+\Phi * \Phi=0
$$

replacing $L_{0}$ with $\mathcal{L}_{0}$ and hoping to find some clues for the ghost number one case. Let us start with an ansatz in the form

$$
\left.\left.\Phi=\sum_{n=0}^{\infty} f_{n} \| n\right\rangle\right\rangle
$$

where we have introduced states

$$
\| n\rangle\rangle=\frac{(-1)^{n}}{2^{n} n !}\left(\mathcal{L}_{0}+\mathcal{L}_{0}^{\dagger}\right)^{n}|0\rangle .
$$

These states appear in the expansion of the wedge states

$$
\left.\left.|r\rangle=\mathrm{e}^{(2-r) / 2\left(\mathcal{L}_{0}+\mathcal{L}_{0}^{\dagger}\right)}|0\rangle=\sum_{n=0}^{\infty}(r-2)^{n}|| n\right\rangle\right\rangle
$$

and can be formally written as

$$
\| n\rangle=\oint \frac{d r}{2 \pi i} \frac{1}{(r-2)^{n+1}}|r\rangle .
$$

We do not pretend here to give meaning to wedge states $|r\rangle$ with complex $r$, we use the residue integral merely as a shorthand for taking derivatives and setting $r=2$. Thanks to the commutation relation $\left[\mathcal{L}_{0}, \mathcal{L}_{0}+\mathcal{L}_{0}^{\dagger}\right]=\mathcal{L}_{0}+\mathcal{L}_{0}^{\dagger}$, 
the states $\| n\rangle\rangle$ are eigenstates of $\mathcal{L}_{0}$

$$
\left.\left.\left.\left.\mathcal{L}_{0}|| n\right\rangle\right\rangle=n \| n\right\rangle\right\rangle
$$

and using $|r\rangle *|s\rangle=|r+s-1\rangle$, one can derive easily their star products

$$
\left.\left.\| n\rangle\rangle * \| m\rangle\rangle=\sum_{k=n+m}^{\infty} \frac{k !}{n ! m !(k-n-m) !} \| k\right\rangle\right\rangle .
$$

The fact that the star product of two states with $\mathcal{L}_{0}$ weights $n$ and $m$ contains weights only greater or equal to $n+m$ is one of the key observations of the present paper that allowed much of the subsequent progress. Strictly speaking, as we have mentioned earlier, the statement is correct only in certain subsector of the string field theory. We will see in the next section that it is fortunately large enough for the physics of tachyon condensation.

Plugging our ansatz to the equation (3.1), we find a simple set of equations

$$
(n-1) f_{n}=-\sum_{\substack{0 \leq p, q \leq n \\ p+q \leq n}} \frac{n !}{p ! q !(n-p-q) !} f_{p} f_{q} .
$$

First equation for $n=0$ is simply $-f_{0}=-f_{0}^{2}$ and requires us to set $f_{0}=1$ or $f_{0}=0$. In the first case, the rest of the coefficients $f_{1}, f_{2}, \ldots$ can be successively and uniquely determined and will be discussed in the next subsection. In the second case one has the freedom to set $f_{1}$ to an arbitrary value. These solutions resemble one parameter pure gauge solutions and we shall comment on them in subsection 3.2.

\section{1 "Tachyon" solutions}

Let us focus on the case $f_{0}=1$ first. Calculating recursively first few coefficients from the equation (3.8), we find $f_{0}=1, f_{1}=-1 / 2, f_{2}=1 / 6, f_{3}=0$, $f_{4}=-1 / 30, \ldots$ Surprisingly, these are nothing but the Bernoulli numbers, so that our solution becomes

$$
\left.\left.\Phi=\sum_{n=0}^{\infty} B_{n} \| n\right\rangle\right\rangle
$$

The Bernoulli numbers $B_{n}$ are one of the most important number sequences in mathematics, with many properties, the most basic ones are for the readers convenience collected in Appendix B. Equation (3.8) appears to be a novel identity for the Bernoulli numbers, somewhat similar to the EulerRamanujan identity. We present an elementary proof in Appendix B.

Having found the solution to (3.1) in the $\| n\rangle\rangle$ basis, we can express it in other forms as well. Using the generating function for the Bernoulli 
numbers (B.1), geometric series expansion, wedge state conservation laws and definition of the Riemann zeta function, we can write it in various forms

$$
\begin{aligned}
\Phi & \left.\left.=\sum_{n=0}^{\infty} B_{n}|| n\right\rangle\right\rangle=\frac{1}{2} \frac{\left(\mathcal{L}_{0}+\mathcal{L}_{0}^{\dagger}\right)}{1-\mathrm{e}^{-1 / 2\left(\mathcal{L}_{0}+\mathcal{L}_{0}^{\dagger}\right)}}|0\rangle \\
& =\frac{1}{2} \sum_{n=0}^{\infty}\left(\mathcal{L}_{0}+\mathcal{L}_{0}^{\dagger}\right) \mathrm{e}^{-n / 2\left(\mathcal{L}_{0}+\mathcal{L}_{0}^{\dagger}\right)}|0\rangle=\frac{1}{2} \sum_{n=0}^{\infty}\left(\mathcal{L}_{0}+\mathcal{L}_{0}^{\dagger}\right)|n+2\rangle \\
& =\sum_{n=0}^{\infty} \frac{1}{n+2} \mathcal{L}_{0}^{\dagger}|n+2\rangle=\sum_{n=0}^{\infty} \frac{1}{2} \mathcal{L}_{0}^{\dagger}\left(\frac{2}{n+2}\right)^{\mathcal{L}_{0}^{\dagger}+1}|0\rangle \\
& =\mathcal{L}_{0}^{\dagger} 2^{\mathcal{L}_{0}^{\dagger}}\left(\zeta\left(\mathcal{L}_{0}^{\dagger}+1\right)-1\right)|0\rangle
\end{aligned}
$$

demonstrating the richness (or perhaps redundancy) of our formalism ${ }^{13}$. From (3.10) and (3.13) we can see that there is formally a term of the form $0 / 0$ or $0 \times \infty$ for $\mathcal{L}_{0}+\mathcal{L}_{0}^{\dagger}$ or $\mathcal{L}_{0}^{\dagger}=0$. One has to be therefore a bit careful. In fact (3.11) and (3.12) cannot be correct, since the expressions are missing the $|0\rangle$ component. The step from (3.10) to (3.11) allows for writing the $\mathcal{L}_{0}+\mathcal{L}_{0}^{\dagger}$ factor inside or outside the sum. If we write it outside the sum, we immediately find using (A.6, A.7) that it acts on

$$
\begin{aligned}
\sum_{n=0}^{\infty}|n+2\rangle= & \sum_{n=0}^{\infty}\left(|0\rangle-\frac{1}{3} L_{-2}|0\rangle+\frac{1}{30} L_{-4}|0\rangle+\frac{1}{18} L_{-2} L_{-2}|0\rangle+\cdots\right) \\
& +\sum_{n=0}^{\infty} \frac{1}{(n+2)^{2}}\left(\frac{4}{3} L_{-2}|0\rangle-\frac{4}{9} L_{-2} L_{-2}|0\rangle+\cdots\right) \\
& +\sum_{n=0}^{\infty} \frac{1}{(n+2)^{4}}\left(-\frac{8}{15} L_{-4}|0\rangle+\frac{8}{9} L_{-2} L_{-2}|0\rangle+\cdots\right)+\cdots
\end{aligned}
$$

All terms here are regular except the first term which is just the sliver state $|\infty\rangle$ found in [54] multiplied by a divergent factor. Acting with $\mathcal{L}_{0}+\mathcal{L}_{0}^{\dagger}$ on $\left(\sum_{n=0}^{\infty} 1\right)|\infty\rangle$ produces an ambiguous answer which has to be fixed to be the sliver state $|\infty\rangle$ itself with unit coefficient. First, it has to be in the kernel of $\mathcal{L}_{0}+\mathcal{L}_{0}^{\dagger}$ and hence proportional to the sliver, and second it has to contain the vacuum $|0\rangle$ with unit coefficient. Adding the sliver to the (3.12)

\footnotetext{
${ }^{13}$ As a side remark, let us note that by expanding $\left.\left.\sum B_{n} \| n\right\rangle\right\rangle$ in the powers of $\mathcal{L}_{0}^{\dagger}$, Stirling numbers of the first kind appear naturally. Comparing this to the same expansion of $\mathcal{L}_{0}^{\dagger} 2^{\mathcal{L}_{0}^{\dagger}}\left(\zeta\left(\mathcal{L}_{0}^{\dagger}+1\right)-1\right)$, we find rather curious relation between the Stieltjes constants $\gamma_{n}$ and products of Bernoulli and Stirling numbers. The sums which appear are only asymptotic series, but they can be summed to arbitrary precision using Padé approximants or exactly via Borel summation.
} 
and trading the $\mathcal{L}_{0}^{\dagger}$ for a derivative with respect to the wedge angle, we can rewrite it in a simple form

$$
\Phi=|\infty\rangle-\left.\sum_{n=2}^{\infty} \frac{d}{d \alpha}|n+\alpha\rangle\right|_{\alpha=0} .
$$

There is actually a much direct connection between the form $\left.\left.\Phi=\sum B_{n} \| n\right\rangle\right\rangle$ and wedge state representation (3.15) which will be useful in the ghost one case. It follows from (2.60) that

$$
\begin{aligned}
\left.\left.\sum_{n=0}^{\infty} B_{n}|| n\right\rangle\right\rangle & =\left.\sum_{n=0}^{\infty} \frac{B_{n}}{n !} \frac{d^{n}}{d r^{n}} U_{r}^{\dagger} U_{r}|0\rangle\right|_{r=2} \\
& =|\infty\rangle-\sum_{n=0}^{\infty} \frac{B_{n}}{n !}\left(\left.\frac{d^{n}}{d r^{n}} U_{r}^{\dagger} U_{r}|0\rangle\right|_{r=\infty}-\left.\frac{d^{n}}{d r^{n}} U_{r}^{\dagger} U_{r}|0\rangle\right|_{r=2}\right) \\
& =|\infty\rangle-\sum_{n=2}^{\infty} \frac{d}{d n}|n\rangle
\end{aligned}
$$

where we used the fact that $U_{r}^{\dagger}|0\rangle=|\infty\rangle+O\left(1 / r^{2}\right)$, and hence all the derivatives $\left(d^{n} / d r^{n}\right) U_{r}^{\dagger}|0\rangle$ vanish at $r=\infty$, except for $n=0$. In the last line, we have used the Euler-Maclaurin sum formula

$$
\sum_{n=0}^{\infty} \frac{B_{n}}{n !}\left[f^{(n)}(b)-f^{(n)}(a)\right]=\sum_{k=a}^{b-1} f^{\prime}(k) .
$$

In practice, the sum formula is most often used in a form (C.2) as a finite sum $n=0, \ldots, N$ with a remainder $R_{N}$. As a series, it is usually rapidly divergent, although there are important exceptions such as polynomials and exponentials. The Euler-Maclaurin series is often Borel summable though, as was shown by Hardy [62]. In the ghost number one case, we will demonstrate for the tachyon coefficient that the Borel summation indeed bridges the solution in the $\mathcal{L}_{0}$ basis written in terms of Bernoulli numbers and a corresponding sum over wedge states.

The form (3.15) is particularly useful for showing that it is indeed a solution of the equations of motion. For the kinetic term, we find

$$
\begin{aligned}
\left(\mathcal{L}_{0}-1\right) \Phi & =-|\infty\rangle-\left.\sum_{n=2}^{\infty} \frac{d}{d \alpha}\left((n+\alpha-2) \frac{d}{d \alpha}-1\right)|n+\alpha\rangle\right|_{\alpha=0} \\
& =-|\infty\rangle-\left.\sum_{n=2}^{\infty}(n-2)\left(\frac{d}{d \alpha}\right)^{2}|n+\alpha\rangle\right|_{\alpha=0},
\end{aligned}
$$


and similarly for the interaction term

$$
\begin{aligned}
\Phi * \Phi & =|\infty\rangle+\left.\sum_{n, m=2}^{\infty} \frac{d}{d \alpha} \frac{d}{d \beta}|n+m+\alpha+\beta-1\rangle\right|_{\alpha=\beta=0} \\
& =|\infty\rangle+\left.\sum_{k=3}^{\infty}(k-2)\left(\frac{d}{d \alpha}\right)^{2}|k+\alpha\rangle\right|_{\alpha=0},
\end{aligned}
$$

which completes our proof. To calculate the term $\left(\mathcal{L}_{0}-1\right) \Phi$, we had to use $\mathcal{L}_{0}|\infty\rangle=0$, which as we discuss in Appendix A.2 is true only if we first regulate the sliver by replacing it with $|r\rangle$ for large $r$, act with $\mathcal{L}_{0}$, do all the normal ordering and take the limit $r \rightarrow \infty$ at the end. Without the regularization, one would encounter divergent sums in the course of normal ordering.

It could seem, therefore, that our solution is not that well behaved after all. Fortunately, this is not the case, as closer inspection of (3.15) reveals. In fact, there is a large cancellation between the two terms in (3.15) at large levels. A simple way to see that is to replace the sum with the integral

$$
\Phi \sim|\infty\rangle-\left.\int_{2}^{\infty} d n \frac{d}{d \alpha}|n+\alpha\rangle\right|_{\alpha=0}=|0\rangle
$$

which is quite good, albeit trivial approximation to the exact solution.

We can confirm the cancellation between the sliver and the sum parts by a more direct computation in the standard Virasoro basis of $L_{0}$ eigenstates. For example, the coefficient of $\left(L_{-2}\right)^{m}|0\rangle$ for the sliver is $(-1)^{m} / 3^{m} m$ !, whereas for the sum part $\sum_{n=2}^{\infty} d /\left.d \alpha|n+\alpha\rangle\right|_{\alpha=0}$ it is

$$
\left.\frac{(-1)^{m}}{3^{m} m !} \sum_{n=2}^{\infty} \frac{d}{d \alpha}\left(1-\frac{4}{(n+\alpha)^{2}}\right)^{m}\right|_{\alpha=0} .
$$

For finite $m$, the sum can be expressed readily in terms of Riemann zeta function and one does not see much signs of cancellation. For $m$ very large, however, this almost infinite sum of Riemann zeta functions can be exactly evaluated up to small corrections

$$
\left.\sum_{n=2}^{\infty} \frac{d}{d \alpha}\left(1-\frac{4}{(n+\alpha)^{2}}\right)^{m}\right|_{\alpha=0}=1+O\left(\mathrm{e}^{-A m^{1 / 3}}\right), \quad A \gtrsim 1.21 .
$$

The error can be rigorously bounded from above by the use of the EulerMaclaurin formula, and the proof is relegated to Appendix C. We thus see almost perfect cancellation between the two terms in (3.15). One could look for similar cancellations for other coefficients, and we have done it also for 
$\left(L_{-4}\right)^{m}|0\rangle$. This time we find the relevant sum to be

$$
\left.\sum_{n=2}^{\infty} \frac{d}{d \alpha}\left(1-\frac{16}{(n+\alpha)^{4}}\right)^{m}\right|_{\alpha=0}=1+O\left(\mathrm{e}^{-B m^{1 / 5}}\right), \quad B \gtrsim 0.80 .
$$

In both cases, there is thus a large cancellation between the sliver and the sum parts of the solution, and that is the reason why the solution has good properties in level truncation.

Let us finish this section by giving an explicit expression for the coefficients in the standard Virasoro basis. From the form (3.15), it is easy to find

$$
\begin{aligned}
\Phi= & |0\rangle+\frac{8 \zeta(3)-9}{3} L_{-2}|0\rangle+\frac{-64 \zeta(5)+65}{30} L_{-4}|0\rangle \\
& +\frac{64 \zeta(5)-16 \zeta(3)-47}{18} L_{-2} L_{-2}|0\rangle+\cdots \\
= & |0\rangle+0.2054 L_{-2}|0\rangle-0.04544 L_{-4}|0\rangle+0.007248 L_{-2} L_{-2}|0\rangle+\cdots
\end{aligned}
$$

Just for comparison, the sliver is

$$
\begin{aligned}
|\infty\rangle & =|0\rangle-\frac{1}{3} L_{-2}|0\rangle+\frac{1}{30} L_{-4}|0\rangle+\frac{1}{18} L_{-2} L_{-2}|0\rangle+\cdots \\
& =|0\rangle-0.3333 L_{-2}|0\rangle+0.03333 L_{-4}|0\rangle+0.005556 L_{-2} L_{-2}|0\rangle+\cdots
\end{aligned}
$$

Although not very obvious from the first four levels, one can easily go to much higher levels to see clearly that the coefficients of $\Phi$ decay much faster than those of the sliver $|\infty\rangle$.

Finally, let us compare our exact solution with a solution obtained by level truncation whose first few coefficients are given in table 1 . The convergence of the level truncation computation is rather slow, presumably due to the fact that in contrast to the equation $\left(L_{0}-1\right) \Phi+\Phi * \Phi=0$, here the level truncation affects the equation itself by approximating $\mathcal{L}_{0}$.

\section{2 "Pure gauge" solutions}

As we mentioned earlier, there is another class of solutions to (3.8) which starts as

$$
\begin{aligned}
& f_{0}=0, \quad f_{1}=\beta, \quad f_{2}=-2 \beta^{2}, \\
& f_{3}=3 \beta^{2}(2 \beta-1), \quad f_{4}=-4 \beta^{2}\left(6 \beta^{2}-6 \beta+1\right), \ldots,
\end{aligned}
$$

where $\beta$ is an arbitrary parameter determining the solution. It looks quite impossible to guess the form of a general term, but the reader may check 


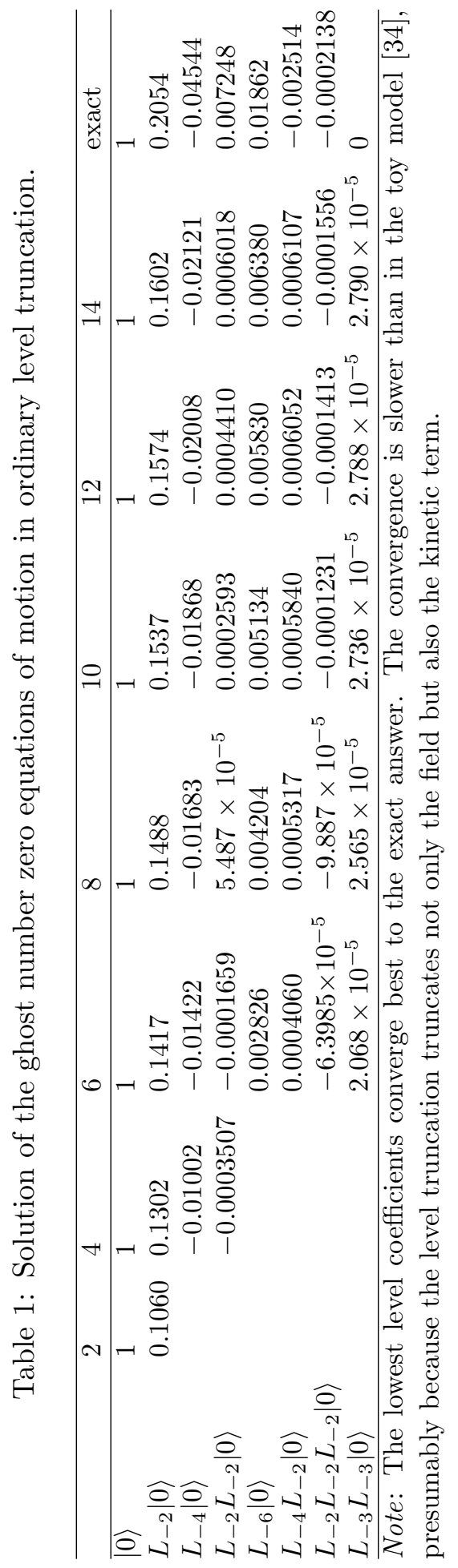


that it is given by

$$
f_{n}=n ! \oint \frac{d z}{2 \pi i} \frac{1}{z^{n+1}} \frac{\lambda z}{\lambda \mathrm{e}^{z}-1}=-n \lambda \mathrm{Li}_{-n+1}(\lambda)-\delta_{n, 1} \lambda,
$$

where $\lambda=\beta / \beta-1$ and $\operatorname{Li}_{n}(z)$ is the polylogarithm function. The solution can be recast in a form similar to (3.15)

$$
\begin{aligned}
\Phi_{\lambda} & =\frac{1}{2} \frac{\left(\mathcal{L}_{0}+\mathcal{L}_{0}^{\dagger}\right) \lambda}{1-\lambda \mathrm{e}^{-1 / 2\left(\mathcal{L}_{0}+\mathcal{L}_{0}^{\dagger}\right)}}|0\rangle \\
& =-\left.\sum_{n=2}^{\infty} \lambda^{n-1} \frac{d}{d \alpha}|n+\alpha\rangle\right|_{\alpha=0},
\end{aligned}
$$

and now it is a rather trivial task to show that (3.28) is a solution, just as we did for (3.15).

Note that since the coefficients of the wedge states are polynomials in $1 / n^{2}$, the solution (3.28) is convergent for $|\lambda| \leq 1$ and hence makes most sense for $\beta \in(-\infty, 1 / 2)$. For $|\lambda|>1$, one attempt could be to use (3.27) to expand around $\lambda=\infty$, but this would generate wedge states with $n=$ $1,0,-1,-2, \ldots$ Although one might think that in some sense $|n\rangle=|-n\rangle$, as is true for the coefficients, the presence of $n=0$ seems to invalidate the expansion. In fact, it follows from the empirical study in [55] that wedge states $|n\rangle$ with $-1<n<1$ are not well behaved in level truncation. In spite of that, it seems that at least some of the values for $|\lambda|>1$ could be meaningful.

For example, for $\lambda=+\infty$ (i.e., $\beta=1$ ), the series truncates after the first term and one finds $\Phi_{\lambda=\infty}=-1 / 2\left(\mathcal{L}_{0}+\mathcal{L}_{0}^{\dagger}\right)|I\rangle=-1 / \pi\left(K_{1}^{L}-K_{1}^{R}\right)|I\rangle$, where $|I\rangle=|1\rangle$ is the identity in the star algebra. ${ }^{14}$ Although this state does not look as ill behaved as wedge states $|n\rangle$ for $n \sim 0$, similar states have been shown to possess anomalous properties [64].

Finally, let us note that for the special value $\lambda=1$, i.e., $\beta=\infty$ we find $\Phi_{\lambda=1}=-\sum_{n=2}^{\infty} \partial_{n}|n\rangle$ which looks just as (3.15) except for the sliver part. It reminds us of the Yang-Mills theory, where the instantons can be viewed as singular limits of pure gauge configurations. In fact, one could regard our toy model "tachyon solution" as a $\lambda \rightarrow 1$ limit of a pure gauge solution if one defines $\Phi_{\lambda}=\lim _{M \rightarrow \infty}\left[\lambda^{M-1}|M\rangle-\sum_{n=2}^{M} \lambda^{n-1} \partial_{n}|n\rangle\right]$ and takes the limit $\lambda \rightarrow 1$ first.

\footnotetext{
${ }^{14}$ This state has been discussed previously in [63]. It was used to show that global symmetries generated by $K_{n}$ can be viewed as part of the gauge symmetry of string field theory.
} 


\section{Ghost number one - the real thing}

Let us now face the real challenge, to solve the ghost number one equation of motion $Q_{B} \Psi+\Psi * \Psi=0$ of the string field theory. As we have anticipated, we will look for solutions in the $\mathcal{B}_{0} \Psi=0$ gauge, where $\mathcal{B}_{0}$ was introduced in (1.12). We shall start by constructing the true vacuum solution in the basis of $\mathcal{L}_{0}$ eigenstates discussed in Section 2.5.

One of the methods used to solve string field equations of motion, which worked in the Siegel gauge, was to use a recursive approach [20]

$$
\psi \rightarrow-\frac{b_{0}}{L_{0}}(\Psi * \Psi)
$$

starting with $\Psi^{(0)} \propto c_{1}|0\rangle .{ }^{15}$ One could hope that the same strategy would work in our new gauge. Starting with $\Psi^{(0)} \propto \tilde{c}_{1}|0\rangle$ and repeatedly star multiplying and acting with $\mathcal{B}_{0} / \mathcal{L}_{0}$ (see (1.16)) leaves us in a simple invariant space which we can parameterize as

$$
\Psi=\sum_{n, p} f_{n, p}\left(\mathcal{L}_{0}+\mathcal{L}_{0}^{\dagger}\right)^{n} \tilde{c}_{p}|0\rangle+\sum_{n, p, q} f_{n, p, q}\left(\mathcal{B}_{0}+\mathcal{B}_{0}^{\dagger}\right)\left(\mathcal{L}_{0}+\mathcal{L}_{0}^{\dagger}\right)^{n} \tilde{c}_{p} \tilde{c}_{q}|0\rangle
$$

where $n=0,1,2, \ldots$, and $p, q=1,0,-1,-2, \ldots$ This will be thus our ansatz for finding the exact solution. Let us now plug the ansatz into the equations of motion $Q_{B} \Psi+\Psi * \Psi=0$. For the coefficient of lowest level ghost number two state $\tilde{c}_{1} \tilde{c}_{0}|0\rangle$, which appears in the equation of motion, we find

$$
f_{0,1}+\pi\left[-\frac{1}{2} f_{0,1}^{2}+f_{0,1}\left(f_{1,1}+2 f_{0,1,0}\right)\right]=0 .
$$

Somewhat unexpectedly, we see that imposing $\mathcal{B}_{0}$ gauge sets $f_{1,1}+2 f_{0,1,0}=$ 0 , the equation can be solved easily, giving two solutions $f_{0,1}=0$ or $f_{0,1}=$ $2 / \pi$. As we discuss further in Appendix E, the first one corresponds to the pure gauge transformation of the vacuum.

Going further to the next level (i.e., the $\mathcal{L}_{0}$ eigenvalue $h=0$ ), we have two states. For $\tilde{c}_{1} \tilde{c}_{-1}|0\rangle$, the equation can be trivially satisfied by the usual requirement of twist invariance which works in our gauge and basis as usual.

\footnotetext{
${ }^{15}$ There are some subtleties to this method, such as the need for adjusting the overall normalization at every step and also tricks to break some peculiar limit cycles. These issues do not affect the present discussion, the interested reader is referred to [20].
} 
For $\left(\mathcal{L}_{0}+\mathcal{L}_{0}^{\dagger}\right) \tilde{c}_{1} \tilde{c}_{0}|0\rangle$, we find

$$
\begin{aligned}
f_{1,1} & +2 f_{0,1,0}+\pi\left[\frac{1}{4} f_{0,1}^{2}-\frac{3}{2} f_{0,1} f_{1,1}-f_{0,1} f_{0,1,0}\right. \\
& \left.+f_{1,1}^{2}+2 f_{1,1} f_{0,1,0}+2 f_{0,1}\left(f_{2,1}+f_{1,1,0}\right)\right]=0 .
\end{aligned}
$$

Imposing the $\mathcal{B}_{0}$ gauge, the equation reduces to

$$
\frac{1}{4} f_{0,1}^{2}-f_{0,1} f_{1,1}=0
$$

which uniquely determines $f_{1,1}=1 / 4 f_{0,1}=1 /(2 \pi)$. Had we chosen in the previous step $f_{0,1}=0$, then $f_{1,1}$ would be a free gauge parameter. It might be surprising that we find pure gauge solutions in our $\mathcal{B}_{0}$ gauge, and the reason is that the state $\left[\left(\mathcal{L}_{0}+\mathcal{L}_{0}^{\dagger}\right) \tilde{c}_{1}-\left(\mathcal{B}_{0}+\mathcal{B}_{0}^{\dagger}\right) \tilde{c}_{1} \tilde{c}_{0}\right]|0\rangle$ is annihilated by all three operators $\mathcal{B}_{0}, Q_{B}$ and $\mathcal{L}_{0}$. There are no other states like this, as one can easily check, since the kernel of $\mathcal{L}_{0}$ at ghost number one is spanned by just three states. ${ }^{16}$

Using the formulas in Appendix D.1, one can show that for any states $\psi_{1}, \psi_{2}$ which satisfy $\mathcal{B}_{0} \psi_{1}=\mathcal{B}_{0} \psi_{2}=0$, their star product obeys

$$
\mathcal{B}_{0}\left(\psi_{1} * \psi_{2}\right)=\frac{\pi}{4}\left(B_{1}^{R} \psi_{1} * \psi_{2}-(-1)^{\operatorname{gh}\left(\psi_{1}\right)} \psi_{1} * B_{1}^{L} \psi_{2}\right) .
$$

Since both $B_{1}^{L}$ and $B_{1}^{R}$ increase $\mathcal{L}_{0}$ eigenvalue by one, the coefficient in front of a state with $\mathcal{L}_{0}=h$ in $\mathcal{B}_{0}\left(\psi_{1} * \psi_{2}\right)$ can receive contributions only from components of $\psi_{1}$ and $\psi_{2}$ with $h_{1}$ and $h_{2}$ such that $h_{1}+h_{2}+1 \leq h$, as we proved in Section 2.5. Acting with $\mathcal{B}_{0}$ on the equation of motion, we have

$$
\mathcal{L}_{0} \Psi+\mathcal{B}_{0}(\Psi * \Psi)=0 .
$$

This equation presents an infinite set of equations, one for each state in the Hilbert space at ghost number one. Let us truncate the equation (but not the string field ) to the subset of states up to some maximal $h$. Then, due to the above identity, and what we have shown above, this truncated system will depend on exactly the right number of coefficients of the string field. This is one of the main advantages of our choice of gauge and basis over traditional Siegel gauge and Virasoro basis.

\footnotetext{
${ }^{16}$ This state is a bit reminiscent of the ghost dilaton $\left(c_{1} c_{-1}-\bar{c}_{1} \bar{c}_{-1}\right)|0\rangle$ in the closed string field theory which is also a $Q_{B}$ exact state annihilated by $b_{0}$, but cannot be written as $Q_{B} \Lambda$ with $b_{0} \Lambda=0$. It would be therefore interesting to study spectrum and interactions of the string field theory around this solution.
} 
Solving our equations to the first two $\mathcal{L}_{0}$ levels, we find

$$
\begin{aligned}
\Psi= & \frac{2}{\pi} \tilde{c}_{1}|0\rangle+\frac{1}{2 \pi}\left[\left(\mathcal{L}_{0}+\mathcal{L}_{0}^{\dagger}\right) \tilde{c}_{1}|0\rangle-\left(\mathcal{B}_{0}+\mathcal{B}_{0}^{\dagger}\right) \tilde{c}_{1} \tilde{c}_{0}|0\rangle\right]+\frac{1}{24 \pi} \\
& \times\left[\left(\mathcal{L}_{0}+\mathcal{L}_{0}^{\dagger}\right)^{2} \tilde{c}_{1}|0\rangle-2\left(\mathcal{B}_{0}+\mathcal{B}_{0}^{\dagger}\right)\left(\mathcal{L}_{0}+\mathcal{L}_{0}^{\dagger}\right) \tilde{c}_{1} \tilde{c}_{0}|0\rangle\right]+\frac{\pi}{48} \tilde{c}_{-1}|0\rangle+\cdots
\end{aligned}
$$

Continuing further becomes rather tedious, so we have written Mathematica program to do it for us, and going to higher levels, we have discovered that all nonzero coefficients at level 12 have factor 691 in the numerator. This number is famous for being the prime numerator of the twelfth Bernoulli number, and so it did not take long to guess the full form of the solution

$$
\begin{aligned}
\Psi= & \sum_{n=0}^{\infty} \sum_{p=-1,1,3,5, \ldots} \frac{\pi^{p}}{2^{n+2 p+1} n !}(-1)^{n} B_{n+p+1}\left(\mathcal{L}_{0}+\mathcal{L}_{0}^{\dagger}\right)^{n} \tilde{c}_{-p}|0\rangle \\
& +\sum_{n=0}^{\infty} \sum_{\substack{p, q=-1 \\
p+q \text { odd }}}^{\infty} \frac{\pi^{p+q}}{2^{n+2(p+q)+3} n !}(-1)^{n+q} B_{n+p+q+2} \\
& \times\left(\mathcal{B}_{0}+\mathcal{B}_{0}^{\dagger}\right)\left(\mathcal{L}_{0}+\mathcal{L}_{0}^{\dagger}\right)^{n} \tilde{c}_{-p} \tilde{c}_{-q}|0\rangle,
\end{aligned}
$$

which we have verified for the first 508 equations with 357 variables. Actually, only 260 equations with 224 variables played role due to the twist symmetry. The details are presented in Appendix E.

Direct proof that (4.9) is a solution of the equation of motion does not seem to be easy. In fact, as we have checked, the proof requires an infinite number of Euler-like identities, like the one in (3.8) proved in Appendix B. Much more convenient starting point for the proof is a form analogous to (3.15):

$$
\Psi=\lim _{N \rightarrow \infty}\left[\psi_{N}-\sum_{n=0}^{N} \partial_{n} \psi_{n}\right]
$$

where $^{17}$

$$
\begin{aligned}
\psi_{n}= & \frac{2}{\pi^{2}} U_{n+2}^{\dagger} U_{n+2}\left[\left(\mathcal{B}_{0}+\mathcal{B}_{0}^{\dagger}\right) \tilde{c}\left(-\frac{\pi}{4} n\right) \tilde{c}\left(\frac{\pi}{4} n\right)\right. \\
& \left.+\frac{\pi}{2}\left(\tilde{c}\left(-\frac{\pi}{4} n\right)+\tilde{c}\left(\frac{\pi}{4} n\right)\right)\right]|0\rangle .
\end{aligned}
$$

\footnotetext{
${ }^{17}$ Let us note equivalent but simpler form $\psi_{n}=1 / \pi c_{1}|0\rangle *\left(B_{1}^{L}-B_{1}^{R}\right)|n\rangle * c_{1}|0\rangle$ for $n \geq 1$. Although it will not play a role in the subsequent analysis, it is worth mentioning that after taking the derivative with respect to $n$ and summing over it, the ghost number zero solution appears naturally. This fact can possibly explain the quasi-pattern found in [34] discussed further in [20]. This new form might be also useful for bringing the solution to the partial isometry or pure-gauge like form advocated in [35].
} 
One could derive that from (4.9) by similar manipulations as in (3.10) or by explicit Borel summation. The easiest way to show the equivalence, however, is to realize that just as in the ghost number zero toy model, the expressions (4.9) and (4.10) are related via Euler-Maclaurin series

$$
\sum_{n=0}^{\infty} \frac{B_{n}}{n !}\left[f^{(n)}(b)-f^{(n)}(a)\right]=\sum_{k=a}^{b-1} f^{\prime}(k)
$$

with $a=0, b=N+1 \rightarrow \infty$ and $f(k)=-\psi_{k}$. To see that, one has to perform the derivatives with the help of formula (2.56). Before we move on to the somewhat involved proof of the equation of motion, we invite the reader to check that (4.10) is actually in the $\mathcal{B}_{0}$ gauge. To see that, one needs only the anticommutator $\left\{\mathcal{B}_{0}, \tilde{c}(\tilde{z})\right\}=\tilde{z}$ and

$$
\mathcal{B}_{0} U_{n+2}^{\dagger} U_{n+2}=U_{n+2}^{\dagger} U_{n+2}\left[\mathcal{B}_{0}-\frac{n}{2}\left(\mathcal{B}_{0}+\mathcal{B}_{0}^{\dagger}\right)\right]
$$

which follows readily from the formulas in Appendix D.1.

\subsection{Proof of the equation of motion}

We shall now give a proof that (4.10) is indeed a solution to the equation of motion $Q_{B} \Psi+\Psi * \Psi=0$. Let us start by ignoring the first term in (4.10) which, as one can readily verify by an explicit calculation, is effectively zero. By that, we mean that all its contractions with Fock space states are zero. It can be also shown using (4.17) that it is irrelevant when star multiplied with itself or the other term in (4.10).

The action of $Q_{B}$ on $\Psi$ is quite simple

$$
\begin{aligned}
Q_{B} \Psi= & -\frac{2}{\pi^{2}} \sum_{n=0}^{\infty} \frac{d}{d n}\left\{U _ { n + 2 } ^ { \dagger } U _ { n + 2 } \left[\left(\mathcal{L}_{0}+\mathcal{L}_{0}^{\dagger}\right) \tilde{c}\left(-\frac{\pi}{4} n\right) \tilde{c}\left(\frac{\pi}{4} n\right)\right.\right. \\
& +\frac{\pi}{2}\left(\tilde{c} \partial \tilde{c}\left(-\frac{\pi}{4} n\right)+\tilde{c} \partial \tilde{c}\left(\frac{\pi}{4} n\right)\right)-\left(\mathcal{B}_{0}+\mathcal{B}_{0}^{\dagger}\right)\left(\tilde{c} \partial \tilde{c}\left(-\frac{\pi}{4} n\right) \tilde{c}\left(\frac{\pi}{4} n\right)\right. \\
& \left.\left.\left.-\tilde{c}\left(-\frac{\pi}{4} n\right) \tilde{c} \partial \tilde{c}\left(\frac{\pi}{4} n\right)\right)\right]\right\}|0\rangle .
\end{aligned}
$$

To calculate the star product $\Psi * \Psi$, it is convenient to rewrite (4.11) as

$$
\begin{aligned}
\psi_{n} & =\frac{2}{\pi} U_{n+2}^{\dagger} U_{n+2}\left[B_{1}^{L} \tilde{c}\left(-\frac{\pi}{4} n\right) \tilde{c}\left(\frac{\pi}{4} n\right)+\tilde{c}\left(-\frac{\pi}{4} n\right)\right]|0\rangle \\
& =\frac{2}{\pi} U_{n+2}^{\dagger} U_{n+2}\left[-B_{1}^{R} \tilde{c}\left(-\frac{\pi}{4} n\right) \tilde{c}\left(\frac{\pi}{4} n\right)+\tilde{c}\left(+\frac{\pi}{4} n\right)\right]|0\rangle .
\end{aligned}
$$


Using the forms (4.15) and (4.16) and the general rules of star multiplication from Section 2, one finds

$$
\begin{aligned}
\psi_{n} * \psi_{m}= & \left(\frac{2}{\pi}\right)^{2} U_{q+2}^{\dagger} U_{q+2}\left[\frac{1}{\pi}\left(\mathcal{B}_{0}+\mathcal{B}_{0}^{\dagger}\right) \tilde{c}\left(\frac{\pi}{4} q\right) \tilde{c}\left(-\frac{\pi}{4} q\right)-\frac{1}{2}\left(\tilde{c}\left(\frac{\pi}{4} q\right)\right.\right. \\
& \left.\left.+\tilde{c}\left(-\frac{\pi}{4} q\right)\right)\right]\left(\tilde{c}\left(\frac{\pi}{4}(r+1)\right)-\tilde{c}\left(\frac{\pi}{4}(r-1)\right)\right)|0\rangle
\end{aligned}
$$

where $q=n+m+1$ and $r=m-n$. It is important to note that the $q$ and $r$ dependent parts are factorized. Moreover, when we re-express the double sum over $n$ and $m$ as

$$
\sum_{n, m=0}^{\infty}=\sum_{q=1}^{\infty} \sum_{\substack{r=-q+1 \\ \text { step2 }}}^{q-1}
$$

we see that summation of (4.17) over $r$ becomes trivial and is given by the first and last terms. Before the summation, we have to, of course, act with $\partial_{m} \partial_{n}=\partial_{q}^{2}-\partial_{r}^{2}$, but that does not spoil this property. Using the identity $\left(\partial^{2} A\right) B-A \partial^{2} B=\partial(A(\overleftarrow{\partial}-\vec{\partial}) B)$, we find

$$
\begin{aligned}
\Psi * \Psi= & \left(\frac{2}{\pi}\right)^{2} \sum_{q=1}^{\infty} \frac{d}{d q}\left\{U _ { q + 2 } ^ { \dagger } U _ { q + 2 } \left[\frac{1}{\pi}\left(\mathcal{B}_{0}+\mathcal{B}_{0}^{\dagger}\right) \tilde{c}\left(\frac{\pi}{4} q\right) \tilde{c}\left(-\frac{\pi}{4} q\right)-\frac{1}{2}\right.\right. \\
& \left.\left.\times\left(\tilde{c}\left(\frac{\pi}{4} q\right)+\tilde{c}\left(-\frac{\pi}{4} q\right)\right)\right]\left(\overleftarrow{\partial}_{q}-\vec{\partial}_{q}\right)\left(\tilde{c}\left(\frac{\pi}{4} q\right)-\tilde{c}\left(-\frac{\pi}{4} q\right)\right)\right\}|0\rangle
\end{aligned}
$$

After a little manipulation, one can bring (4.18) to the form of (4.14) with a minus sign, and noting that the $n=0$ term of (4.14) actually vanishes, the equation of motion $Q_{B} \Psi+\Psi * \Psi=0$ is proven.

\subsection{Proof of Sen's first conjecture}

Now we are going to prove Sen's first conjecture using the explicit form of the solution (4.10). Sen's $[11,12]$ first conjecture states that the energy density of the true vacuum found by solving the open string field theory equations of motion should be equal to minus the tension of the D25 brane i.e., $-1 /\left(2 \pi^{2} g_{o}^{2}\right)$. The energy density of a static configuration is minus the action, ${ }^{18}$ so we are going to prove

$$
V(\Psi)=\frac{1}{g_{o}^{2}}\left[\frac{1}{2}\left\langle\Psi, Q_{B} \Psi\right\rangle+\frac{1}{3}\langle\Psi, \Psi * \Psi\rangle\right]=-\frac{1}{2 \pi^{2} g_{o}^{2}}
$$

\footnotetext{
${ }^{18}$ Since we are interested in translationally invariant solutions, we normalize our correlators such that they do not depend on the volume of the space. In other words, we are setting $V_{25}=1$ and we are not distinguishing between the energy and its density.
} 
Since $\Psi$ is a solution of the equations of motion, all we have to show is that

$$
\left\langle\Psi, Q_{B} \Psi\right\rangle=-\frac{3}{\pi^{2}} \text {. }
$$

Using the correlators from Appendix D.2, we find

$$
\begin{aligned}
\left\langle\psi_{n}, Q_{B} \psi_{m}\right\rangle= & \frac{1}{\pi^{2}}\left(1+\cos \left(\frac{\pi r}{p}\right)\right)\left(-1+\frac{p}{\pi} \sin \left(\frac{2 \pi}{p}\right)\right) \\
& +2 \sin ^{2}\left(\frac{\pi}{p}\right)\left[-\frac{p-1}{\pi^{2}}+\frac{(p-2)^{2}-r^{2}}{4 \pi^{2}} \cos \left(\frac{\pi r}{p}\right)\right. \\
& \left.+\frac{p r}{2 \pi^{3}} \sin \left(\frac{\pi r}{p}\right)\right],
\end{aligned}
$$

where we have introduced $p=m+n+2$ and $r=m-n$. We also find

$$
\begin{aligned}
\left\langle\partial_{n} \psi_{n}, Q_{B} \partial_{m} \psi_{m}\right\rangle= & -\frac{4(p-1)}{p^{4}} \cos \left(\frac{2 \pi}{p}\right)+\frac{1}{8 p^{4}}\left[f_{p}(r+2)-f_{p}(r)\right. \\
& \left.+f_{p}(-r+2)-f_{p}(-r)\right],
\end{aligned}
$$

where we denote

$$
f_{p}(r)=-\left((p-2)^{2}-(r-2)^{2}\right)\left(p^{2}-r^{2}\right) \cos \left(\frac{\pi r}{p}\right) .
$$

Let us re-express the double sum as

$$
\sum_{n, m=0}^{\infty}=\sum_{p=2}^{\infty} \sum_{\substack{r=-p+2 \\ \text { step2 }}}^{p-2}
$$

and observe that the special structure of (4.22) with the help of (4.23) gives readily

$$
\sum_{\substack{r=-p+2 \\ \text { step2 }}}^{p-2}\left\langle\partial_{n} \psi_{n}, Q_{B} \partial_{m} \psi_{m}\right\rangle=0 .
$$

This is, of course, welcome since it shows that the energy of pure gauge solutions $\Psi_{\lambda}=-\sum_{n=0}^{\infty} \lambda^{n+1} \partial_{n} \psi_{n}$ is manifestly zero. But it also shows that if one carelessly interpreted (4.10) as $-\sum_{n=0}^{\infty} \partial_{n} \psi_{n}$, one would find zero energy, at least with the above order of summation. In fact, one could find arbitrary result since the double sum $\sum_{n, m=0}^{\infty}\left\langle\partial_{n} \psi_{n}, Q_{B} \partial_{m} \psi_{m}\right\rangle$ is not absolutely convergent.

In our case, however, the sum is properly regularized (4.10) and there is thus no ambiguity left. We would like to stress that the regularization (4.10) is in no way ad-hoc but was imposed on us by the use of the Euler-Maclaurin formula and confirmed by the analogy with ghost number zero toy model. In the next two subsections, we shall provide two other rather orthogonal numerical verifications, which give the same energy with high precision. 
With our regularization, we thus have

$$
\begin{aligned}
\left\langle\Psi, Q_{B} \Psi\right\rangle= & \lim _{N \rightarrow \infty}\left[\left\langle\psi_{N}, Q_{B} \psi_{N}\right\rangle-2 \sum_{m=0}^{N}\left\langle\psi_{N}, Q_{B} \partial_{m} \psi_{m}\right\rangle\right. \\
& \left.+\sum_{n=0}^{N} \sum_{m=0}^{N}\left\langle\partial_{n} \psi_{n}, Q_{B} \partial_{m} \psi_{m}\right\rangle\right] .
\end{aligned}
$$

For the first term, one readily finds from (4.21)

$$
\lim _{N \rightarrow \infty}\left\langle\psi_{N}, Q_{B} \psi_{N}\right\rangle=\frac{1}{2}+\frac{2}{\pi^{2}}
$$

For the third term, we rewrite the sum over the square $(n, m) \in[0, N] \times$ $[0, N]$ as a sum over the lower left and upper right triangles, i.e.,

$$
\sum_{n=0}^{N} \sum_{m=0}^{N}=\sum_{p=2}^{N+2} \sum_{\substack{r=-p+2 \\ \text { step } 2}}^{p-2}+\sum_{p=N+3}^{2 N+2} \sum_{\substack{r=-2 N+p-2 \\ \text { step } 2}}^{2 N-p+2} .
$$

The first double sum does not contribute by (4.24), the second one gives

$$
\begin{aligned}
& \sum_{p=N+3}^{2 N+2} \sum_{\substack{r=-2 N+p-2 \\
\text { step } 2}}^{2 N-p+2}\left\langle\partial_{n} \psi_{n}, Q_{B} \partial_{m} \psi_{m}\right\rangle \\
& =\sum_{j=1}^{N} \frac{4}{(2+j+N)^{4}}\left[\left(j^{2}-(N+1)^{2}\right) \cos \left(\frac{2 \pi}{2+j+N}\right)+\left(j^{2}-1\right)(N+1)^{2}\right. \\
& \left.\quad \times \cos \left(\frac{\pi(j-N)}{2+j+N}\right)+j^{2} N(N+2) \cos \left(\frac{2 j \pi}{2+j+N}\right)\right]
\end{aligned}
$$

Note that for every fixed $j$, the summand on the right hand side goes as $16 \pi^{2}\left(j^{3}-j\right) / N^{4}$ for large $N$. The dominant contribution comes therefore from large $j$ 's. Let us introduce $x=j / N$ and expand the summand in $1 / N$ keeping $x \in(0,1]$ fixed

$$
\frac{8 \pi x^{2}}{(1+x)^{5}} \sin \left(\pi \frac{1-x}{1+x}\right) \frac{1}{N}+O\left(\frac{1}{N^{2}}\right) .
$$

Since the sum involves $N$ bounded terms, we can safely ignore the $O\left(1 / N^{2}\right)$ part, and the sum of the first term is in the limit nothing but the Riemann 
definition of an integral. Therefore,

$$
\begin{aligned}
\lim _{N \rightarrow \infty}\left[\sum_{n=0}^{N} \sum_{m=0}^{N}\left\langle\partial_{n} \psi_{n}, Q_{B} \partial_{m} \psi_{m}\right\rangle\right]= & \int_{0}^{1} d x \frac{8 \pi x^{2}}{(1+x)^{5}} \sin \left(\pi \frac{1-x}{1+x}\right) \\
& =\frac{1}{2}-\frac{1}{\pi^{2}}
\end{aligned}
$$

Similarly, for the middle term in (4.25), we find using (4.21) and $\partial_{m}=$ $\partial_{p}+\partial_{r}$ expression where we set $p=N(1+x)+2, r=(x-1) N$. Expanding in $N$ keeping $x=m / N$ fixed, we find again the Riemann integral

$$
\lim _{N \rightarrow \infty}\left[\sum_{m=0}^{N}\left\langle\psi_{N}, Q_{B} \partial_{m} \psi_{m}\right\rangle\right]=\int_{0}^{1} d x \frac{4 \pi x}{(1+x)^{4}} \sin \left(\pi \frac{1-x}{1+x}\right)=\frac{1}{2}+\frac{2}{\pi^{2}} .
$$

Altogether

$$
\left\langle\Psi, Q_{B} \Psi\right\rangle=\frac{1}{2}+\frac{2}{\pi^{2}}-2\left(\frac{1}{2}+\frac{2}{\pi^{2}}\right)+\frac{1}{2}-\frac{1}{\pi^{2}}=-\frac{3}{\pi^{2}},
$$

which completes our proof of Sen's first conjecture.

\subsection{Transforming to the Virasoro basis}

In this section, we would like to demonstrate that our solution (4.10) is a well-behaved element of the string field theory Hilbert space, just like the Siegel gauge solution found by Sen and Zwiebach [5] in their seminal paper. We shall not delve here into the issue of regularity in the string field theory. Instead, we shall give first few coefficients of the solution in the standard Virasoro basis, which is the one used in level truncation.

As we have already mentioned, the first term in (4.10) does not contribute in level truncation, and thus, with a little manipulation, we arrive to a convenient, almost normal ordered form

$$
\begin{aligned}
\Psi= & -\frac{1}{\pi} \sum_{n=2}^{\infty} \frac{d}{d n}\left\{U _ { n } ^ { \dagger } \left[\frac{n}{\pi} \mathcal{B}_{0}^{\dagger} \tilde{c}\left(-\frac{\pi}{2} \frac{n-2}{n}\right) \tilde{c}\left(\frac{\pi}{2} \frac{n-2}{n}\right)\right.\right. \\
& \left.\left.+\tilde{c}\left(-\frac{\pi}{2} \frac{n-2}{n}\right)+\tilde{c}\left(\frac{\pi}{2} \frac{n-2}{n}\right)\right]\right\} .
\end{aligned}
$$

Using the explicit form of the canonically ordered wedge state (2.20), see also Appendix A.1, and the definitions of $\mathcal{B}_{0}^{\dagger}$ and $\tilde{c}$, we easily derive all the 
coefficients at low levels. Just for illustration, let us write the exact solution up to level 4 following the notation of Sen and Zwiebach:

$$
\begin{aligned}
\Psi= & t c_{1}|0\rangle+u c_{-1}|0\rangle+v L_{-2} c_{1}|0\rangle+w b_{-2} c_{0} c_{1}|0\rangle \\
& +A L_{-4} c_{1}|0\rangle+B L_{-2} L_{-2} c_{1}|0\rangle+C c_{-3}|0\rangle+D b_{-3} c_{-1} c_{1}|0\rangle \\
& +E b_{-2} c_{-2} c_{1}|0\rangle+F L_{-2} c_{-1}|0\rangle+w_{1} L_{-3} c_{0}|0\rangle \\
& +w_{2} b_{-2} c_{-1} c_{0}|0\rangle+w_{3} b_{-4} c_{0} c_{1}|0\rangle+w_{4} L_{-2} b_{-2} c_{0} c_{1}|0\rangle .
\end{aligned}
$$

For the first four coefficients (level 0 and level 2), we find

$$
\begin{aligned}
t & =\sum_{n=2}^{\infty} \frac{d}{d n}\left[\frac{n}{\pi} \sin ^{2}\left(\frac{\pi}{n}\right)\left(-1+\frac{n}{2 \pi} \sin \left(\frac{2 \pi}{n}\right)\right)\right] \\
u & =\sum_{n=2}^{\infty} \frac{d}{d n}\left[\left(\frac{4}{n \pi}-\frac{n}{\pi} \sin ^{2}\left(\frac{\pi}{n}\right)\right)\left(-1+\frac{n}{2 \pi} \sin \left(\frac{2 \pi}{n}\right)\right)\right] \\
v & =\sum_{n=2}^{\infty} \frac{d}{d n}\left[\left(\frac{4}{3 n \pi}-\frac{n}{3 \pi}\right) \sin ^{2}\left(\frac{\pi}{n}\right)\left(-1+\frac{n}{2 \pi} \sin \left(\frac{2 \pi}{n}\right)\right)\right] \\
w & =\sum_{n=2}^{\infty} \frac{d}{d n}\left[\sin ^{2}\left(\frac{\pi}{n}\right)\left(\frac{8}{3 n \pi}-\frac{2 n}{3 \pi}+\frac{n^{2}}{3 \pi^{2}} \sin \left(\frac{2 \pi}{n}\right)\right)\right] .
\end{aligned}
$$

These sums do not appear to have simple analytic expressions, although they can be rewritten in an interesting way using the Bernoulli numbers. We can simply expand the trigonometric functions into their Taylor series and exchange the two infinite sums to find fast converging sums such as

$$
\begin{aligned}
t & =\sum_{k=2}^{\infty}(-1)^{k}(2 \pi)^{2 k-1} \frac{(2 k-1)\left(2^{2 k}-2 k-2\right)}{(2 k+1) !} \zeta(2 k) \\
& =\sum_{k=2}^{\infty}(2 \pi)^{4 k-1} \frac{(2 k-1)\left(k+1-2^{2 k-1}\right)}{(2 k) !(2 k+1) !} B_{2 k} .
\end{aligned}
$$

For practical purposes, one can keep the sums (4.34) as they are, since all summands behave as $1 / n^{4}$ for large $n$ and can be easily evaluated numerically with arbitrary precision. ${ }^{19}$

We have computed the exact coefficients with nine digit precision up to level 10, and some numerical results are given in Appendix F. ${ }^{20}$ Let us

\footnotetext{
${ }^{19}$ To speed up the convergence, it is convenient to sum first explicitly given number of terms (e.g., first one hundred), expand the remaining terms in powers of $1 / n$ keeping only first few orders and sum them exactly using the Riemann zeta function. Note that in the $1 / n$ expansion, only terms $1 / n^{4}, 1 / n^{6}, 1 / n^{8}, \ldots$, appear.

${ }^{20}$ To be completely honest, at level 10 , due to inefficiency of our computer program, we left our solution expressed in terms of $L_{n}^{\text {tot }}$ and the ghosts, instead of $L_{n}^{\text {matter }}$ and the
} 
present here the complete list of the exact coefficients up to level 4 :

\begin{tabular}{llll}
\hline$t=0.55346558$ & $A=-0.030277583$ & $E=0.17942652$ & $w_{1}=0$ \\
$u=0.45661043$ & $B=0.0045805832$ & $F=0.022748278$ & $w_{2}=0.020943544$ \\
$v=0.13764616$ & $C=-0.16494614$ & & $w_{3}=0.088982260$ \\
$w=-0.14421001$ & $D=0.16039444$ & $w_{4}=-0.0084696519$
\end{tabular}

One thing one can do with these coefficients is to check whether the solution obeys the expected symmetries. From the explicit form (4.10), (4.11) or (4.32), one sees that $K_{1}^{\text {matter }} \Psi=0$, since $K_{1}^{\text {matter }}$ commutes with all the terms, including the common factor $U_{n}^{\dagger} U_{n}$. It is a general rule that if a solution of $Q_{B} \Psi+\Psi * \Psi=0$ is annihilated by a star algebra derivative $D$, it must be annihilated also by $\left[Q_{B}, D\right]_{ \pm}$. For the case at hand, it is easy to check

$$
\begin{aligned}
5 A+3 B+v & =0 \\
w_{1} & =0 \\
20 A+12 B+4 D-4 F-8 w_{1} & =0 \\
15 A+9 B+v+w-10 w_{1}+5 w_{3}+3 w_{4} & =0
\end{aligned}
$$

as dictated by $K_{1}^{\text {matter }} \Psi=\left[K_{1}^{\text {matter }}, Q_{B}\right] \Psi=0$. Actually one can see those identities to be true also from the explicit expressions (4.34) before the sums are carried out.

In the Siegel gauge, there is a somewhat unexpected $S U(1,1)$ symmetry $[20,31,32,65]$, which implies $(c \partial c)_{0} \Psi=0$. Note that $(c \partial c)_{0}$ is a star algebra derivative whose commutator with $Q_{B}$ is zero. This invariance enforces the Siegel gauge $b_{0} \Psi=0$. In addition, it implies a constraint $C+3 D=0$ and even more constraints at higher levels. It is definitely of some interest to see whether our $\mathcal{B}_{0}$ gauge solution possesses similar symmetries. Given the fact that we have expressions like (4.34), we can look for such symmetries systematically. Surprisingly, we have found one more independent identity

$$
2 A+4 D-3 E+2 F-3 w_{2}+3 w_{4}=0 .
$$

We were not able to find any simple origin, it might be just an accidental symmetry. Apart from $K_{1}^{\text {matter }} \Psi=\left[K_{1}^{\text {matter }}, Q_{B}\right] \Psi=0$, there is one more obvious symmetry $K_{1} B_{1} \mathcal{B}_{0}^{\dagger} \Psi=0$ which gives some exact constraints manifest in level truncation, but they become nontrivial only at level 6 . To complete the discussion of symmetries, we remind the reader at this point

ghosts. This does not affect the check on the $D$-brane energy as given below, which we were primarily interested in. 
of the obvious twist symmetry

$$
(-1)^{L_{0}-1} \Psi=\Psi
$$

which we in fact imposed when solving the equations of motion in the $\mathcal{L}_{0}$ basis and which our solution shares with the Siegel gauge solution. Finally, there is yet another symmetry, which as far as we can see, is obvious only from the solution (4.9) in the $\mathcal{L}_{0}$ basis. All the terms with $\mathcal{L}_{0}$ eigenvalue equal to $m$ are multiplied by the Bernoulli number $B_{m+1}$. Now, all odd index Bernoulli numbers vanish except $B_{1}$, and it turns out that the term multiplied by $B_{1}$ is annihilated by all three operators $Q_{B}, \mathcal{B}_{0}$ and $\mathcal{L}_{0}$. We can thus write the symmetry as

$$
(-1)^{\mathcal{L}_{0}-1} \mathcal{L}_{0} \Psi=\mathcal{L}_{0} \Psi
$$

It would be interesting to see if it can be translated to the Virasoro basis.

One slight disadvantage of the $\mathcal{B}_{0}$ gauge is that the gauge fixing condition is broken by level truncation. As we noted earlier, by virtue of (4.13), the solution indeed obeys $\mathcal{B}_{0} \Psi=0$ exactly, but after truncating it to level 4 , the gauge conditions become

$$
\begin{gathered}
w_{i}=0, \quad i=1,2,3,4 \\
\frac{2}{3} E+w=0,
\end{gathered}
$$

out of which only $w_{1}=0$ is true exactly. The last condition is true within $83 \%$, and for $w_{2,3,4}$, we can only say that they are two to three times smaller than similar coefficients without $c_{0}$. We hope that this level dependent gauge fixing would not pose problems and that the numerical high level computations of Moeller and Taylor [19] and Gaiotto and Rastelli [20] would converge to our solution. On the other hand, we do not expect convergence properties superior to the Siegel gauge because of our experience with the ghost number zero equation discussed in Section 3.

Finally we would like to demonstrate that our solution yields the correct D25-brane energy density also in level truncation. Note that this is the main problem with the identity based solutions [38]. To check the energy, we have evaluated the kinetic term $\left\langle\Psi, Q_{B} \Psi\right\rangle$ up to level 10 . The values are summarized in table 2 .

Table 2: Energy density normalized by the $D$-brane tension at various levels of truncation of the exact solution. The numbers which appear are $\left\langle\Psi, Q_{B} \Psi\right\rangle$ divided by $3 / \pi^{2}$.

\begin{tabular}{llllll}
\hline$L=0$ & $L=2$ & $L=4$ & $L=6$ & $L=8$ & $L=10$ \\
-1.007766 & -1.007815 & -1.004499 & -1.003217 & -1.002556 & -1.002130 \\
\hline
\end{tabular}




\subsection{Padé approximants and Borel summation}

Instead of passing through the representation in terms of wedge states or using level truncation one could attempt to compute the energy density or coefficients in the Virasoro basis directly from the tachyon solution (4.9) written in terms of Bernoulli numbers. As we shall see, both tasks lead to divergent series, but ones which can be handled with. Let us start by "regularizing" our solution by replacing $\Psi$ with $z^{\mathcal{L}_{0}} \Psi$. In the $\mathcal{L}_{0}$ level expansion, different levels will acquire different integer powers of $z$. The "regularization" is then removed in the limit $z \rightarrow 1$. We have put regularization in quotation marks, since as we shall see, $z$ does not quite regularize the energy nor the Virasoro coefficients but merely provides an expansion parameter for an asymptotic series.

\subsubsection{Energy}

Let us start with the computation of the energy as a formal expansion in $z$. Using the explicit solution (4.9) and few correlators from Appendix D.2, we arrive to ${ }^{21}$

$$
\begin{aligned}
\left\langle\Psi, z^{\mathcal{L}_{0}^{\dagger}} Q_{B} z^{\mathcal{L}_{0}} \Psi\right\rangle & \\
= & -\frac{4}{\pi^{2} z^{2}}+\left(\frac{1}{12}+\frac{1}{3 \pi^{2}}\right)-\left(\frac{1}{90}+\frac{\pi^{2}}{1920}\right) z^{2}+\left(\frac{17}{5040}-\frac{11 \pi^{2}}{17920}\right. \\
& \left.-\frac{\pi^{4}}{193536}\right) z^{4}+\left(-\frac{113}{60480}+\frac{2413 \pi^{2}}{1935360}-\frac{137 \pi^{4}}{5806080}-\frac{\pi^{6}}{22118400}\right) z^{6} \\
& +\cdots
\end{aligned}
$$

Trying to evaluate the series numerically for $z=1$, one immediately finds that the series is divergent. The most common method for dealing numerically with divergent series is the Padé approximation. This, a bit mysterious, but often a very successful method approximates a series outside its radius of convergence by a rational function. Given a formal power series $f(z) \sim \sum a_{n} z^{n}$, Padé approximant $P_{M}^{N}(z)$ is a ratio of two polynomials of degree $N$ and $M$, such that its power series matches the one of $f(z)$ up to $z^{M+N}$. In table 3 , we give a Padé approximation $P_{n+2}^{n}$ for even $n=0, \ldots, 18$ and compare it with the naive evaluation which can be viewed as $P_{2}^{2 n}$. Note that both $P_{n+2}^{n}$ and $P_{2}^{2 n}$ match (4.46) to the same order.

\footnotetext{
${ }^{21}$ The closed form expression we found for the series contains six-fold sum of a product of two Bernoulli numbers, six factorials (five of them in the denominator) and some powers of 2 and $\pi$. We did not dare to simplify it; however, we noticed that at given order of $z^{n}$ the term with the highest power of $\pi$ simplifies to $-1 /(2(n+2) n !)\left|B_{n+2}\right|(\pi / 2)^{n}$ for $n \geq 1$. There is an easy proof which uses the Euler identity (B.4).
} 
Table 3: The Padé approximation for the normalized energy $\pi^{2} / 3\left\langle\Psi, z^{\mathcal{L}_{0}^{\dagger}} Q_{B} z^{\mathcal{L}_{0}} \Psi\right\rangle$ evaluated at $z=1$.

\begin{tabular}{lll}
\hline & $P_{2}^{2 n}$ & $P_{n+2}^{n}$ \\
\hline$n=0$ & -1.3333 & -1.33333333 \\
$n=2$ & -1.0015 & -0.99501646 \\
$n=4$ & -0.98539 & -1.00100097 \\
$n=6$ & -1.0327 & -1.00032831 \\
$n=8$ & -1.3054 & -1.00042520 \\
$n=10$ & 6.7582 & -1.00003423 \\
$n=12$ & 256.34 & -0.99999846 \\
$n=14$ & -21575. & -0.99999945 \\
$n=16$ & $3.6391 \times 10^{6}$ & -0.99999819 \\
$n=18$ & $6.5671 \times 10^{7}$ & -1.00000064 \\
\hline
\end{tabular}

The first column is in fact a trivial approximation, a naively summed series with behavior typical for asymptotic series.

The second column nicely confirms Sen's first conjecture despite somewhat irregular convergence at higher orders.

The first column with $P_{2}^{2 n}$ is also nothing but the definition of the energy in the $\mathcal{L}_{0}$-level truncation. Interestingly, we see that with level 2 , we get very close to the exact value and up to level 6 we are still within a few percent. At higher levels, the divergent character of the series starts to show up. ${ }^{22}$

Given the relative ease of evaluating (4.46), we carried out the expansion up to $z^{50}$, and to our surprise, we found that to this order the Padé approximations do not improve much beyond the $10^{-6}$ accuracy. Looking separately at the contribution of the first term in (4.9) only, we found that the convergence is rather irregular, with a rough pattern of plateaux of constant accuracy and occasional bigger jumps towards better accuracy. It seems that to reach accuracy of $10^{-9}$, one would need at least a Padé approximant $P_{52}^{50}$. The somewhat irregular convergence is in sharp contrast with the behavior of other series, such as the celebrated Euler series $\sum(-1)^{n} n ! z^{n}$ or $\sum B_{n} z^{n}$ (for which we know the exact answer by the Borel summation), and where we checked that the Padé approximants converge to the exact answer monotonically.

\footnotetext{
${ }^{22}$ This raises the unwelcome possibility that ordinary $L_{0}$-level truncation in the Siegel gauge would show up a similar behavior, perhaps at some higher level $\gtrsim 20$. The overshooting of the correct energy at level 14 found by Gaiotto and Rastelli [20] could be attributed to it. It is not clear to us whether the high level extrapolations of $[20,66]$ resolve the issue.
} 


\subsubsection{Tachyon coefficient}

Let us now see how one can get the tachyon coefficient $t$ directly from (4.9). As in the case of the energy, we "regularize" our solution by considering $z^{\mathcal{L}_{0}} \Psi$ and the tachyon coefficient will become $z$ dependent. Let us write $t=t_{1}+t_{2}$ for the two contributions coming from the two terms in (4.9). Using (D.14) from Appendix D.2, one finds

$$
\begin{aligned}
t_{1}(z)= & \sum_{n=0}^{\infty} \sum_{\substack{p=-1 \\
p \text { odd }}}^{\infty} z^{n+p} \frac{(-1)^{n}}{n !}\left(\frac{\pi}{2}\right)^{p} \frac{B_{n+p+1}}{2^{n+p+1}}(p-1)_{n} \\
& \times\left[\frac{2^{p}}{(p+1) !}(-1)^{(p+1) / 2}+\frac{1}{2} \delta_{p,-1}\right], \\
t_{2}(z)= & \sum_{n=0}^{\infty} \sum_{\substack{p=-1 \\
p \text { odd }}}^{\infty} \sum_{q=0}^{\infty} z^{n+p+q+1} \frac{(-1)^{n+q}}{n !}\left(\frac{\pi}{2}\right)^{p+q} \frac{B_{n+p+q+2}}{2^{n+p+q+2}}(p+q)_{n} \\
& \times\left[\frac{2^{p}}{(p+1) !}(-1)^{(p+1) / 2}+\frac{1}{2} \delta_{p,-1}\right]\left[\frac{2^{q}}{(q+1) !}(-1)^{(q+2) / 2}-\delta_{q, 0}\right],
\end{aligned}
$$

which as one can easily check are again divergent series due to the presence of Bernoulli numbers and the Pochhammer symbol $(x)_{n}=x(x+1) \cdots(x+$ $n-1)$. Before attempting the Borel summation, which may not always work and often requires some labor, we propose as a rule of thumb to check the series first with Padé approximants. ${ }^{23}$ We have found that the Padé approximants $P_{N}^{N}$ to $t_{1,2}(z)$ evaluated at $z=1$ approach the expected values

$$
\begin{aligned}
t_{1} & =\frac{\pi}{2}+\sum_{k=1}^{\infty}(-1)^{k}(2 \pi)^{2 k+1} \frac{k}{(2 k+2) !} \zeta(2 k+1)=0.277658977 \cdots \\
t_{2} & =-t_{1}+\sum_{k=1}^{\infty}(-1)^{k}(2 \pi)^{2 k-1} \frac{(2 k-1)\left(2^{2 k}-2 k-2\right)}{(2 k+1) !} \zeta(2 k) \\
& =0.275806609 \cdots
\end{aligned}
$$

in a similar manner as the energy or perhaps a bit faster. Originally, we found these exact expressions by performing the Borel summation, but they can be most easily derived from (4.10) and (4.11). Note that the first term in (4.10) contributes $\pi / 2$ in (4.49) and $-\pi / 2$ in (4.50) inside $-t_{1}$. Although it is not true, in general, that the Padé approximation to a sum of functions is a sum of their Padé approximations, we see that the sum of Padé approximants to $t_{1}$ and $t_{2}$ at $z=1$ approaches the correct value $t=0.553465587 \ldots$ One

\footnotetext{
${ }^{23}$ There is actually a theorem that under certain conditions both the Borel summation and the Padé approximation lead to the same result. I thank J. Fischer for a discussion on this issue.
} 
could perform the Padé approximation directly for the sum $t_{1}+t_{2}$ with the same results, although for finite $N$ the results differ.

Let us now sketch how one can perform the Borel summation for the series (4.47) and (4.48). This was actually our first computation of $t$ before we discovered the simple representation in terms of wedge states. First, observe that both expressions (4.47) and (4.48) contain a common part which can be summed separately for $r \geq 2$ and $\operatorname{Re} z>0$

$$
\begin{aligned}
\sum_{n=0}^{\infty}(r-2)_{n} \frac{B_{n+r}}{n !}\left(\frac{z}{2}\right)^{n+r-1}= & (-1)^{r}\left[\frac{(r-1)(r-2)}{z}+\frac{r-2}{2}+\frac{z}{12}\right. \\
& \left.-\sum_{j=0}^{r-3}\left(\frac{2}{z}\right)^{j+2} \frac{r ! \psi_{j+1}(2 / z)}{j !(j+3) !(r-j-3) !}\right] .
\end{aligned}
$$

In this formula $\psi_{n}(z)=(-1)^{n+1} n ! \sum_{k=0}^{\infty}(z+k)^{-n-1}$ denotes the polygamma function. For lower values $r=0$ and $r=1$, the sum on the left hand side terminates and one finds $2 z^{-1}+1+z / 12$ and $-(z+6) / 12$, respectively. Using this result, one can readily derive

$$
\begin{aligned}
& t_{1}(z)=\frac{\pi}{2 z}+\sum_{k=1}^{\infty}(-1)^{k}\left(\frac{2 \pi}{z}\right)^{2 k+1} \frac{k}{(2 k+2) !} \zeta\left(2 k+1, \frac{2}{z}\right) \\
& t_{2}(z)=-t_{1}(z)+\frac{1}{z} \sum_{k=1}^{\infty}(-1)^{k}\left(\frac{2 \pi}{z}\right)^{2 k-1} \frac{(2 k-1)\left(2^{2 k}-2 k-2\right)}{(2 k+1) !} \zeta\left(2 k, \frac{2}{z}\right),
\end{aligned}
$$

where $\zeta(n, z)=\sum_{k=0}^{\infty}(k+z)^{-n}$ is the Hurwitz zeta function. For the sum $t(z)=t_{1}(z)+t_{2}(z)$, we find finally

$$
\begin{aligned}
t(z) & =+\frac{1}{z} \sum_{k=1}^{\infty}(-1)^{k}\left(\frac{2 \pi}{z}\right)^{2 k-1} \frac{(2 k-1)\left(2^{2 k}-2 k-2\right)}{(2 k+1) !} \zeta\left(2 k, \frac{2}{z}\right) \\
& =\sum_{n=0}^{\infty} \frac{d}{d(n z)}\left[\frac{2+n z}{\pi} \sin ^{2}\left(\frac{\pi}{2+n z}\right)\left(-1+\frac{2+n z}{2 \pi} \sin \left(\frac{2 \pi}{2+n z}\right)\right)\right] .
\end{aligned}
$$

Let us make few comments. The easiest way to obtain (4.55) is by using the wedge state representation (4.10) and (4.11). Note that the action of $z^{\mathcal{L}_{0}}$ effectively replaces all factors of $n$ with $n z$. From (4.54) for $z=1$ follows immediately (4.35), one has to use the identity $\zeta(n, 2)=\zeta(n)-1$ and observe that the term -1 does not contribute. Finally observe that the function $t(z)$ is holomorphic at $z=\infty$ (the functions $t_{1}(z)$ and $t_{2}(z)$ have 
first order poles there which cancel each other). On the contrary, it has an essential singularity at $z=0$ as can be seen from (4.55), since $z=0$ is a cumulation point of essential singularities at $z=-2 / n$. This explains why the series (4.47) and (4.48) have zero radius of convergence.

\section{Conclusions and outlook}

We have found the first exact and fully explicit nonsingular solution describing the nonperturbative tachyon vacuum in Witten's cubic open bosonic string field theory. We also definitely proved Sen's first conjecture, which relates the value of the tachyon potential at the minimum to the $D$-brane tension known from the annulus computation. Good evidence was presented that our solution is quite regular from the point of view of level truncation. It would be interesting to confirm it by direct numerical computations.

We have presented our solution in two different forms. In the first form, the solution is written in the basis of $\mathcal{L}_{0}$ eigenstates and is given in terms of Bernoulli numbers. In this basis, it was rather straightforward to find the solution, although it was not easy to prove that it actually is a solution. Another advantage of this basis is that it is rather easy to study exactly a large sector of the full infinite-dimensional gauge symmetry and thus clearly discriminate the tachyon solution from pure gauge solutions.

The second form can be most elegantly obtained from the first one by noticing that it is an Euler-Maclaurin series of certain sum over wedge states with ghost insertions. For this form of the solution, it was fairly easy to prove that it solves the equations of motion and that it obeys Sen's first conjecture.

Clearly now we are at a stage where many new exciting things can be done. There are still two other Sen's conjectures that remain to be proved. We believe that with the tools developed in this paper, the cohomology of the kinetic operator at the vacuum can be studied rather easily and hopefully shown to be empty. Studying space-time-dependent solutions, such as higher codimension $D$-branes or rolling tachyon backgrounds, could also be possible with the presented methods, although the presence of nontrivial contractions among matter operators makes their study a challenge. We hope that one could also study the question of how closed strings emerge at the tachyon vacuum. It seems very likely that our techniques could be used for efficient computation of off-shell string amplitudes in the $\mathcal{B}_{0}$ gauge, which would be much simpler than those in the Siegel gauge. 
In this paper, we focussed solely on the open bosonic string field theory. It seems quite possible that our methods extend to the Berkovits superstring field theory, since it is based on Witten's associative star product. On the other hand, for the closed string field theory [59], we are much less optimistic because of the multitude of higher order vertices and especially because of the level matching condition $b_{0}^{-} \Psi=0$, which does not fit well into our algebraic framework. So far, all attempts to eliminate the level matching condition or put it on the same footing as a gauge choice have been unsuccessful.

\section{Acknowledgments}

I am grateful to Ian Ellwood for collaboration at the initial stages of this work. I am indebted to Barton Zwiebach for useful discussions and especially for detailed reading of the manuscript, which helped clarify many issues and improve the presentation. I would also like to thank organizers of Benasque Center for Science workshop on string theory for providing lovely environment while this work was in progress.

\section{Appendix A Comments on surface states}

\section{A.1 Representation in terms of Virasoro operators}

The wedge states discussed extensively in Section 2 are a prime example of more general surface states $[16,54]$. The surface states are in one-to-one correspondence with conformal maps $f(z)$ holomorphic inside the unit disk $|z|<1$. They are defined by the relation

$$
\langle f \mid \phi\rangle=\langle f \circ \phi\rangle, \quad \forall \phi .
$$

In the operator formalism, they can be expressed as $\langle f|=\langle 0| U_{f}$, where $U_{f}=$ $\exp \left(\sum v_{n} L_{n}\right)$ is an exponential of non-negatively moded Virasoro generators. The coefficients $v_{n}$ can be thought of as Laurent coefficients of a vector field $v(z)=\sum v_{n} z^{n+1}$, which is related to the map $f(z)$ by the Julia equation $v(z) \partial_{z} f(z)=v(f(z))$. In practice, given $v(z)$, the equation is fairly easy to integrate to find $f(z)$ [67], the inverse problem is much harder and usually one has to resort to an iterative procedure to determine the coefficients of the vector field. One class of solutions [58] is particularly useful. They are the maps

$$
f_{n, t}(z)=\frac{z}{\left(1-t n z^{n}\right)^{1 / n}}
$$


generated by a vector field $v(z)=t z^{n+1}$, so that $U_{f_{n, t}}=\mathrm{e}^{t L_{n}}$. These maps played a pivotal role recently in the study of butterfly projectors [67-69] within the context of vacuum string field theory.

Apart of their importance for the butterfly projectors, these maps can be taken as some kind of a basis for holomorphic maps. Any map $f(z)$ vanishing at the origin and holomorphic in its neighborhood can be uniquely decomposed as

$$
f(z)=f_{0, t_{0}} \circ f_{1, t_{1}} \circ f_{2, t_{2}} \circ \ldots
$$

In a sense, this is a complete parametrization of the space of conformal maps holomorphic at the origin. ${ }^{24}$ Given a power series expansion around the origin, this decomposition is unique. It can be easily implemented on a computer since $f_{n, t}=z+t z^{n+1}+O\left(z^{2 n+1}\right)$. The decomposition (A.3) is useful because using the composition rule $U_{f \circ g}=U_{f} U_{g}$ (reflecting the fact that $U_{f}$ form a representation of the conformal group), the operator $U_{f}$ can be written as

$$
U_{f}=\mathrm{e}^{t_{0} L_{0}} \mathrm{e}^{t_{1} L_{1}} \mathrm{e}^{t_{2} L_{2}} \cdots .
$$

For the surface states $\langle f|=\langle 0| U_{f}$, the first two exponentials are of course irrelevant. Expanding the other exponentials in powers of $L_{n}$ yields automatically canonically ordered form

$$
\langle f|=\sum_{k_{2}, k_{3}, k_{4}, \ldots} \frac{t_{2}^{k_{2}} t_{3}^{k_{3}} t_{4}^{k_{4}} \cdots}{k_{2} ! k_{3} ! k_{4} ! \cdots}\langle 0| L_{2}^{k_{2}} L_{3}^{k_{3}} L_{4}^{k_{4}} \cdots,
$$

which is very useful in level truncation. This decomposition was found to take very simple form for the identity state [25] and also for the "nothing state" projector in $[67,69]$.

For the purposes of the present paper, where we use at several occasions the level truncation to check and illustrate certain exact computations, we need a decomposition (A.4) for the wedge states. With the help of a computer, we easily find for $f_{r}=\tan (2 / r \arctan z)$

$$
U_{r} \equiv U_{f_{r}}=\left(\frac{2}{r}\right)^{L_{0}} \mathrm{e}^{u_{2} L_{2}} \mathrm{e}^{u_{4} L_{4}} \mathrm{e}^{u_{6} L_{6}} \mathrm{e}^{u_{8} L_{8}} \mathrm{e}^{u_{10} L_{10}} \cdots
$$

\footnotetext{
${ }^{24}$ There is also another, as far as we can see unrelated, parameterization of this space using harmonic moments which can be thought of as times of dispersionless Toda hierarchy. This has been applied to the study of wedge states [70] and of the three-vertex [71].
} 
where the coefficients $u_{n}$ are given by

$$
\begin{aligned}
u_{2} & =-\frac{r^{2}-4}{3 r^{2}} \\
u_{4} & =\frac{r^{4}-16}{30 r^{4}} \\
u_{6} & =-\frac{16\left(r^{2}-4\right)\left(r^{2}-1\right)\left(r^{2}+5\right)}{945 r^{6}} \\
u_{8} & =\frac{\left(r^{2}-4\right)\left(109 r^{6}+436 r^{4}-944 r^{2}+1344\right)}{11340 r^{8}} \\
u_{10} & =-\frac{16\left(r^{2}-4\right)\left(r^{2}-1\right)\left(9 r^{6}+45 r^{4}-64 r^{2}+160\right)}{22275 r^{10}} .
\end{aligned}
$$

Note that all the coefficients vanish for $r=2$, i.e., the vacuum, but also $u_{6}=u_{10}=0$ for $r=1$ in accord with the observation of Ellwood et al. [25].

\section{A.2 Conservation laws}

Conservation laws in the string field theory are quite a useful tool. We use them to tell us what is the action of a given mode of an arbitrary operator on a surface state or any kind of $n$-vertex. They were first studied systematically by Rastelli and Zwiebach in [54], although some of them appeared in the literature much earlier. In what follows, we will be mainly interested in the so-called Virasoro conservation laws associated with the energy-momentum tensor, and for simplicity, we shall assume zero central charge.

The basic conservation laws for arbitrary surface state $\langle f|=\langle 0| U_{f}$ can be written trivially as

$$
\langle f| f^{-1} \circ L_{-n}=0
$$

since $f^{-1} \circ L_{-n}=U_{f}^{-1} L_{-n} U_{f}$ and $L_{-n}$ annihilates the vacuum $\langle 0|$. In the language of [54], one would write

$$
\langle f| \oint v^{w}(w) T_{w w}(w) d w=0
$$

for any vector field in the global coordinate $w$ that is holomorphic everywhere including infinity except possibly the puncture. Transforming to the local 
coordinate $z$ gives

$$
\langle f| \oint v^{z}(z) T_{z z}(z) d z=0 .
$$

Using the transformation law for the vector field $v^{z}(z)=\left(f^{\prime}(z)\right)^{-1} v^{w}(w)$, one finds conservation laws

$$
\langle f| \oint \frac{d w}{2 \pi i} \frac{f(w)^{-n+1}}{f^{\prime}(w)} \sum_{m=-n}^{\infty} \frac{L_{m}}{w^{m+2}}=0,
$$

which are identical to (A.8). An example of such conservation laws for the sliver was given in $(2.40)$.

The disadvantage of this form of conservation laws is that it expresses an action of an operator $L_{n}$ for $n \geq-1$ not as a sum of operators $L_{k}$ with $k \leq-2$ acting on $|f\rangle$ but it involves also operators $L_{k}$ with $-2<k<n$, for which one would like to use the conservation laws again. It is clearly desirable to have more direct way of writing the conservation laws one needs.

There is actually a trick to do that. The key observation is that the $b(z)$ ghost has the same conservation laws as the energy-momentum tensor $T(z)$ with zero central charge. For the $b$ ghost, the conservation laws in the right form follow readily from the Neumann matrix representation. Thus, the task is reduced to find the Neumann matrix such that in the ghost sector

$$
\left\langle\left. f\right|_{\text {ghost }}=\langle 0| \mathrm{e}^{\sum c_{p} S_{p q} b_{q}} .\right.
$$

There is a simple way to do it. ${ }^{25}$ We can simply evaluate the correlator

$$
\left\langle f\left|b_{-n} c_{-m} c \partial c \partial^{2} c(0)\right| 0\right\rangle
$$

in two different ways and match the results. Using the fact that $\langle f|=\langle 0| U_{f}$ and performing the conformal transformation on the ghosts, we find

$$
\left\langle f\left|b(z) c(w) c \partial c \partial^{2} c(0)\right| 0\right\rangle=\frac{\left(f^{\prime}(z)\right)^{2}}{f^{\prime}(w)}\left(\frac{f(w)}{f(z)}\right)^{3} \frac{2}{f(w)-f(z)},
$$

and therefore

$$
\begin{aligned}
\left\langle f\left|b_{-n} c_{-m} c \partial c \partial^{2} c(0)\right| 0\right\rangle= & \oint \frac{d z}{2 \pi i} \oint \frac{d w}{2 \pi i} \frac{1}{z^{n-1}} \frac{1}{w^{m+2}} \frac{\left(f^{\prime}(z)\right)^{2}}{f^{\prime}(w)} \\
& \times\left(\frac{f(w)}{f(z)}\right)^{3} \frac{2}{f(w)-f(z)} .
\end{aligned}
$$

On the other hand, using the normalization $\left\langle c \partial c \partial^{2} c(0)\right\rangle=-2$ (i.e., $\left.\left\langle c_{-1} c_{0} c_{1}\right\rangle=1\right)$, we get

$$
\left\langle 0\left|\mathrm{e}^{\sum c_{p} S_{p q} b_{q}} b_{-n} c_{-m} c \partial c \partial^{2} c(0)\right| 0\right\rangle=2 S_{n m}
$$

\footnotetext{
${ }^{25}$ I thank Barton Zwiebach for suggesting the method.
} 
and hence

$$
S_{n m}=\oint \frac{d z}{2 \pi i} \oint \frac{d w}{2 \pi i} \frac{1}{z^{n-1}} \frac{1}{w^{m+2}} \frac{\left(f^{\prime}(z)\right)^{2}}{f^{\prime}(w)}\left(\frac{f(w)}{f(z)}\right)^{3} \frac{1}{f(w)-f(z)} .
$$

The conservation laws then read

$$
\begin{aligned}
\langle f| b_{n} & =-\sum_{m=2}^{\infty} S_{n m}\langle f| b_{-m}, \\
\langle f| L_{n} & =-\sum_{m=2}^{\infty} S_{n m}\langle f| L_{-m} .
\end{aligned}
$$

One application where we used the conservation laws (A.19) was to test the frequently used conservation law for the wedge states

$$
\mathcal{L}_{0}|r\rangle=\frac{2-r}{r} \mathcal{L}_{0}^{\dagger}|r\rangle
$$

which follows directly from (2.57). This conservation law can be also derived following Rastelli and Zwiebach using a vector field

$$
v^{w}(w)=2\left(1+w^{2}\right) \operatorname{arccot} w
$$

This vector field is not globally defined but is holomorphic everywhere outside the unit circle including the infinity, so that (A.9) still holds for a contour encircling the infinity. By deforming the contour onto the unit circle and passing to the local coordinate, one finds

$$
v^{z}(z)=(r-2)\left(1+z^{2}\right) \arctan z+r\left(1+z^{2}\right) \operatorname{arccot} z
$$

from which

$$
\langle r|\left(r \mathcal{L}_{0}^{\dagger}+(r-2) \mathcal{L}_{0}\right)=0
$$

follows, and hence also (A.20). Although we have derived or proved (A.20) in many ways, we wanted to see whether it really works in level truncation. Using (A.19), we calculated the $L_{-2}$ coefficient of $\mathcal{L}_{0}|r\rangle$ and compared with the expected result $8(2-r) / 3 r^{3} L_{-2}|0\rangle+\cdots$ from the right hand side of (A.20). The numerical agreement turned out to be quite good for finite $r$, but for $r$ set to infinity $\mathcal{L}_{0}|\infty\rangle$ did not seem to converge, although formally it can be set to zero. This only stresses the importance of the observation made in Section 3 and further discussed in Appendix $\mathrm{C}$ that the sliver and the sum part of (3.15) cancel each other to a large extent. 
OPEN STRING FIELD THEORY

\section{Appendix B Bernoulli numbers}

The Bernoulli numbers are among the most important number sequences in number theory. They are defined through

$$
\frac{x}{\mathrm{e}^{x}-1}=\sum_{n=0}^{\infty} \frac{B_{n} x^{n}}{n !}
$$

The first few non-trivial numbers are $B_{0}=1, B_{1}=-1 / 2, B_{2}=1 / 6$, $B_{4}=-1 / 30, B_{6}=1 / 42, B_{8}=-1 / 30, B_{10}=5 / 66, B_{12}=-691 / 2730, \ldots$. The obey a number of remarkable properties, and the most basic ones are

$$
\begin{aligned}
B_{2 k+1} & =0, \quad \forall k \geq 1 \\
\sum_{k=0}^{n} \frac{B_{k}}{k !} \frac{1}{(n+1-k) !} & =0, \quad \forall n \geq 1 .
\end{aligned}
$$

Well known is also the Euler identity

$$
(n+1) B_{n}=-\sum_{k=2}^{n-2} \frac{n !}{k !(n-k) !} B_{k} B_{n-k}, \quad \forall n \geq 3 .
$$

There are number of other linear, quadratic or higher order identities [72]. It appears however that the ones we have discovered by solving the string field theory equations of motion were previously unknown. The first one is quite similar to the Euler identity

$$
(n-1) B_{n}=-\sum_{\substack{0 \leq p, q \leq n \\ p+q \leq n}} \frac{n !}{p ! q !(n-p-q) !} B_{p} B_{q}, \quad \forall n \geq 0 .
$$

A simple proof using (B.3) goes as follows. Let us write the right hand side of (B.5) as

$$
\begin{aligned}
- & B_{n}-\sum_{q=0}^{n-1} \sum_{p=0}^{n-q} \frac{n !(n-p-q+1)}{p ! q !(n-p-q+1) !} B_{p} B_{q} \\
= & -B_{n}+\sum_{q=0}^{n-1} \sum_{p=1}^{n-q} \frac{n !}{(p-1) ! q !(n-p-q+1) !} B_{p} B_{q}= \\
& -B_{n}+\sum_{p=1}^{n} \sum_{q=0}^{n-p} \frac{n !}{(p-1) ! q !(n-p-q+1) !} B_{p} B_{q}=(n-1) B_{n} .
\end{aligned}
$$

In the first sum, only the $-p$ term from the factor $(n-p-q+1)$ in the numerator was contributing, thanks to (B.3). In the last sum, only $p=$ $n, q=0$ term was contributing, thanks to the same identity. 
Another important fact about Bernoulli numbers we need is their asymptotics

$$
B_{2 k}=2(-1)^{k-1} \frac{(2 k) !}{(2 \pi)^{2 k}} \zeta(2 k)=2(-1)^{k-1} \frac{(2 k) !}{(2 \pi)^{2 k}}\left(1+O\left(2^{-2 k}\right)\right) .
$$

\section{Appendix C Proof of the sum-sliver cancellation}

In this appendix, we shall apply the Euler-Maclaurin formula to establish rigorous lower bound on positive constant $A_{p}$ such that

$$
\left.\sum_{k=2}^{\infty} \frac{d}{d \alpha}\left[1-\left(\frac{2}{k+\alpha}\right)^{p}\right]^{M}\right|_{\alpha=0}=1+O\left(\mathrm{e}^{-A_{p} M^{1 /(p+1)}}\right) .
$$

This in turn will imply the estimates (3.21) and (3.22), proving thus the cancellation between the two terms in $|\infty\rangle-\sum_{n=2}^{\infty} \partial_{n}|n\rangle$ for two classes of high level coefficients, namely $\left(L_{-2}\right)^{M}|0\rangle$ and $\left(L_{-4}\right)^{M}|0\rangle$.

The Euler-Maclaurin formula states that (see e.g., [73,74])

$$
\sum_{k=a}^{b-1} f(k)=\sum_{n=0}^{N} \frac{B_{n}}{n !}\left[f^{(n-1)}(b)-f^{(n-1)}(a)\right]+R_{N},
$$

where $B_{n}$ are the Bernoulli numbers (see Appendix B), and by $f^{(-1)}$ we denote the primitive function $\int^{t} f(t) d t$. The remnant $R_{N}$ for arbitrary $N$ is given by

$$
R_{N}=\frac{1}{N !} \int_{a}^{b} B_{N}(t-[t]) f^{(N)}(t) d t
$$

where $B_{n}(x)$ are the Bernoulli polynomials and $[t]$ denotes the integer part of $t$. For a given function, the Euler-Maclaurin formula is typically useful only up to certain maximal $N$ which minimizes the error. This is because of the eventual factorial growth of the Bernoulli numbers and polynomials. ${ }^{26}$

Applying the formula (C.2) to our sum and taking the harmless limit $b \rightarrow$ $\infty$, we see that for $0<n \leq N<M$, the $(n-1)$-th derivatives of our function $f(t)=\partial_{t}\left[1-(2 / t)^{p}\right]^{M}$ all vanish at $t=2$ and $t=\infty$. Thus, it is only the first term in (C.2) with $n=0$ and the remnant $R_{N}$ which contribute. For

\footnotetext{
${ }^{26}$ The Euler-Maclaurin formula written for infinite $N$ without a remnant in most cases presents an asymptotic series which can be summed via Borel summation technique [62]. The cases when the series converges by itself are rare and the most prominent examples are polynomials and exponentials.
} 
the upper bound on the remnant, one can use $\left|B_{N}(x)\right| \leq\left|B_{N}\right|$ for $x \in[0,1]$ for $N$ even and hence

$$
\left|R_{N}\right| \leq \frac{\left|B_{N}\right|}{N !} \int_{2}^{\infty}\left|f^{(N)}(t)\right| d t
$$

The strategy is now to find a value of $N, 0<N<M$ such that $\left|R_{N}\right|$ is minimized. For that, we need an accurate estimate of $\int_{2}^{\infty}\left|f^{(N)}(t)\right| d t$, which is actually the hardest part of the proof.

Naive expansion of $d^{N+1} / d t^{N+1}\left[1-(2 / t)^{p}\right]^{M}$ into the binomial series, taking the absolute value of each term and integrating it, would not work. The estimate would be too crude and useless, since it would not take into account that at $t=2$ the integrand vanishes.

Let us start with the formula for derivative of a composite function

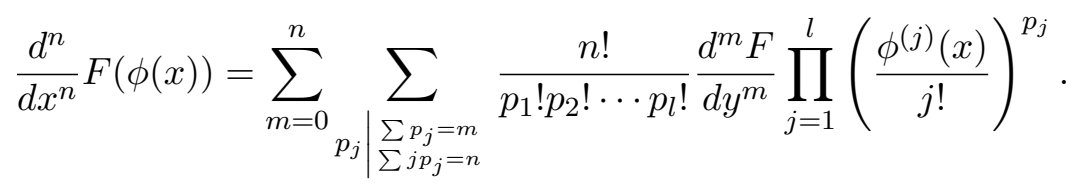

Inserting the identity in the forms of $1=\oint d z /(2 \pi i) 1 / z^{n+1} \prod_{j} z^{j p_{j}}$ and $1=$ $\oint d w /(2 \pi i) 1 / w^{m+1} \prod_{j} w^{p_{j}}$ and performing the sum over all $p_{j}$ 's, we find

$$
\frac{d^{n}}{d x^{n}} F(\phi(x))=n ! \sum_{m=0}^{n} \frac{d^{m} F}{d y^{m}} \oint \frac{d z}{2 \pi i} \frac{1}{z^{n+1}} \frac{1}{m !}[\phi(x+z)-\phi(x)]^{m} .
$$

Let us set $F(y)=y^{M}, \phi(x)=1-(2 / x)^{p}$ and assume $n>0$. Then by using $\phi(x+z)-\phi(x)=(2 / x)^{p}\left[1-(1+z / x)^{-p}\right]$ and with the help of binomial expansion for the $m$-th power, we find easily by direct integration

$$
\begin{aligned}
\int_{2}^{\infty}\left|\frac{d^{n}}{d x^{n}}\left[1-\left(\frac{2}{x}\right)^{p}\right]^{M}\right| \leq & \frac{M ! 2^{-n+1} p^{-1}}{\Gamma(M+(n-1) / p+1)} \sum_{m=1}^{n} \sum_{k=1}^{m} \\
& \times \frac{\Gamma(m+(n-1) / p)(p k+n-1) !}{k !(m-k) !(p k-1) !} .
\end{aligned}
$$

The double sum on the right hand side can be replaced by the maximal term times a factor of $n^{2}$ which is not going to affect the leading behavior. The maximum is achieved for $m=n$ and $k=c_{p} n$, where $c_{p}$ is a solution to

$$
\left(1+\frac{1}{p c_{p}}\right)^{p}=\frac{c_{p}}{1-c_{p}}
$$

i.e., $c_{2}=0.738, c_{4}=0.758$ for the cases of interest. Now setting $n=N+1$, using $B_{N} / N ! \sim(2 \pi)^{-N}$, we can minimize the remnant. The minimum is 
attained for $n \propto M^{1 /(p+1)}$, and by calculating the exact coefficient, we find

$$
\left|R_{N}\right| \leq K_{p} \mathrm{e}^{-A_{p} M^{1 /(p+1)}}
$$

where $K_{p}$ is some finite constant and $A_{p}=\left(4 \pi\left(1-c_{p}\right) /\left(1+p c_{p}\right)\right)^{p /(p+1)}$. Again, for the cases of interest, we find $A_{2}=1.210$ and $A_{4}=0.799$, which seem to be smaller by a factor of four from what numerical fits would suggest. To obtain more precise estimate of $A_{p}$ and not just upper bound would be more challenging, since $B_{N}(t-[t])$ is a periodic function and large cancellations in (C.3) are taking place. Anyway it is nice that apart of proving an upper bound, we were able to capture the qualitative behavior, i.e., the power of $M^{1 /(p+1)}$ in the exponent.

\section{Appendix D Collection of useful formulas}

\section{D.1 $\mathcal{B}_{0}$-gauge formulas}

We define

$$
\begin{aligned}
\mathcal{B}_{0} & =\oint \frac{d \tilde{z}}{2 \pi i} \tilde{z} b_{\tilde{z} \tilde{z}}(\tilde{z})=\tan \circ b_{0}=b_{0}+\sum_{k=1}^{\infty} \frac{2(-1)^{k+1}}{4 k^{2}-1} b_{2 k} \\
& =b_{0}+\frac{2}{3} b_{2}-\frac{2}{15} b_{4}+\cdots \\
\mathcal{B}_{0}^{\dagger} & =\oint \frac{d \tilde{z}}{2 \pi i} \tilde{z} b_{\tilde{z} \tilde{z}}\left(\tilde{z}-\frac{\pi}{2}\right)=b_{0}+\sum_{k=1}^{\infty} \frac{2(-1)^{k+1}}{4 k^{2}-1} b_{-2 k} \\
& =b_{0}+\frac{2}{3} b_{-2}-\frac{2}{15} b_{-4}+\cdots \\
B_{1} & =\oint_{\frac{d \tilde{z}}{2 \pi i}} b_{\tilde{z} \tilde{z}}(\tilde{z})=b_{1}+b_{-1} \\
B_{1}^{L} & =\oint_{C_{L}} \frac{d \tilde{z}}{2 \pi i} b_{\tilde{z} \tilde{z}}(\tilde{z})=\frac{1}{2} B_{1}+\frac{1}{\pi}\left(\mathcal{B}_{0}+\mathcal{B}_{0}^{\dagger}\right) \\
B_{1}^{R} & =\oint_{C_{R}} \frac{d \tilde{z}}{2 \pi i} b_{\tilde{z} \tilde{z}}(\tilde{z})=\frac{1}{2} B_{1}-\frac{1}{\pi}\left(\mathcal{B}_{0}+\mathcal{B}_{0}^{\dagger}\right),
\end{aligned}
$$

where the open contours $C_{L}$ and $C_{R}$ are the left half (Re $\left.\tilde{z}>0\right)$ and the right half $(\operatorname{Re} \tilde{z}<0)$ of the unit circle. These objects clearly satisfy $B_{1}^{L}+B_{1}^{R}=$ $B_{1}$ and further

$$
\begin{aligned}
B_{1}^{L}\left(\phi_{1} * \phi_{2}\right) & =\left(B_{1}^{L} \phi_{1}\right) * \phi_{2}, \\
B_{1}^{R}\left(\phi_{1} * \phi_{2}\right) & =(-1)^{\operatorname{gn}\left(\phi_{1}\right)} \phi_{1} *\left(B_{1}^{R} \phi_{2}\right), \\
B_{1}\left(\phi_{1} * \phi_{2}\right) & =\left(B_{1} \phi_{1}\right) * \phi_{2}+(-1)^{\operatorname{gn}\left(\phi_{1}\right)} \phi_{1} *\left(B_{1} \phi_{2}\right) .
\end{aligned}
$$


The first two equations are manifestations of the fact that Witten's star product glues together the right part of the first string with the left part of the second string, so that $B_{1}^{L}$ acting on the first string can be pulled out of the product. Same is true for $B_{1}^{R}$ on the second string with appropriate Grassman sign. The last equation tells us that $B_{1}$ is a graded derivation of the star algebra.

We also frequently need to commute $\mathcal{B}_{0}^{\dagger}$ through the operator $U_{r}=(2 / r)^{\mathcal{L}_{0}}$ or $\mathcal{B}_{0}$ through $U_{r}^{\dagger}$

$$
\begin{aligned}
U_{r} \mathcal{B}_{0}^{\dagger} U_{r}^{-1} & =\frac{2-r}{r} \mathcal{B}_{0}+\frac{2}{r} \mathcal{B}_{0}^{\dagger}, \\
U_{r}^{\dagger-1} \mathcal{B}_{0} U_{r}^{\dagger} & =\frac{2}{r} \mathcal{B}_{0}+\frac{2-r}{r} \mathcal{B}_{0}^{\dagger}, \\
U_{r}^{-1} \mathcal{B}_{0}^{\dagger} U_{r} & =\frac{r-2}{2} \mathcal{B}_{0}+\frac{r}{2} \mathcal{B}_{0}^{\dagger}, \\
U_{r}^{\dagger} \mathcal{B}_{0} U_{r}^{\dagger-1} & =\frac{r}{2} \mathcal{B}_{0}+\frac{r-2}{2} \mathcal{B}_{0}^{\dagger} .
\end{aligned}
$$

Useful anticommutators are

$$
\begin{aligned}
\left\{\mathcal{B}_{0}, \tilde{c}(\tilde{z})\right\} & =\tilde{z} \\
\left\{B_{1}, \tilde{c}(\tilde{z})\right\} & =1 .
\end{aligned}
$$

\section{D.2 Some correlators}

Using the definitions $\tilde{c}(x)=\cos ^{2}(x) c(\tan x)$ and the fact that inversion acts simply as a translation $I \circ \tilde{c}(x)=\tilde{c}(x-\pi / 2)=\tilde{c}(x+\pi / 2)$, we readily derive ${ }^{27}$

$$
\begin{aligned}
\langle\tilde{c}(x) \tilde{c}(y) \tilde{c}(z)\rangle & =\sin (x-y) \sin (x-z) \sin (y-z), \\
\langle I \circ \tilde{c}(x) \tilde{c}(y) \tilde{c}(z)\rangle & =\cos (x-y) \cos (x-z) \sin (y-z), \\
\langle I \circ \tilde{c}(x) I \circ \tilde{c}(y) \tilde{c}(z)\rangle & =\sin (x-y) \cos (x-z) \cos (y-z), \\
\langle I \circ \tilde{c}(x) I \circ \tilde{c}(y) I \circ \tilde{c}(z)\rangle & =\sin (x-y) \sin (x-z) \sin (y-z), \\
\langle\tilde{c}(x) \tilde{c} \partial \tilde{c}(y)\rangle & =-\sin (x-y)^{2}, \\
\langle I \circ \tilde{c}(x) \tilde{c} \partial \tilde{c}(y)\rangle & =-\cos (x-y)^{2} .
\end{aligned}
$$

\footnotetext{
${ }^{27}$ All the correlators are taken on the UHP. Also, it would be more consistent with our previous notation if all $x, y$ and $z$ had a tilde.
} 
Useful correlators involving the $\mathcal{B}_{0}+\mathcal{B}_{0}^{\dagger}$ operator are

$$
\begin{aligned}
& \left\langle I \circ \tilde{c}(x) I \circ \tilde{c}(-x)\left(\mathcal{B}_{0}+\mathcal{B}_{0}^{\dagger}\right) \tilde{c}(y) \tilde{c}(-y)\right\rangle \\
& =2 y \sin (2 x) \cos (x+y) \cos (x-y)+2 x \sin (2 y) \cos (x+y) \cos (x-y) \\
& \left\langle I \circ \tilde{c}(x) I \circ \tilde{c}(-x)\left(\mathcal{B}_{0}+\mathcal{B}_{0}^{\dagger}\right) \tilde{c} \partial \tilde{c}(y)\right\rangle \\
& =-x\left(\cos ^{2}(x+y)+\cos ^{2}(x-y)\right)+\left(y \partial_{y}-1\right) \sin (2 x) \cos (x-y) \\
& \quad \times \cos (x+y) .
\end{aligned}
$$

Evaluating the above correlators for particular modes is not necessarily a simple task. We can use $\left\langle c_{-1} c_{0} c_{1}\right\rangle=1$ and

$$
\begin{aligned}
\tilde{c}_{-2 k} & =(-1)^{k} \frac{2^{2 k}}{(2 k+1) !} c_{0}+\cdots, \\
\tilde{c}_{-(2 k-1)} & =(-1)^{k} \frac{2^{2 k}}{(2 k) !} \frac{c_{1}-c_{-1}}{2}+\delta_{k, 0} \frac{c_{1}+c_{-1}}{2}+\cdots,
\end{aligned}
$$

where the dots indicate modes other than $c_{-1}, c_{0}$ and $c_{1}$. We then find

$$
\left\langle\left(\tilde{c}_{-p}\right)^{\dagger} Q_{B} \tilde{c}_{-q}\right\rangle=\frac{2^{p+q+1}}{(p+1) !(q+1) !}(-1)^{(p+q) / 2}-\frac{1}{2} \delta_{p,-1} \delta_{q,-1} .
$$

Assuming $p_{1}$ and $q_{1}$ to be odd and $p_{2}$ and $q_{2}$ to be even, we find further

$$
\begin{gathered}
\left\langle\left(\tilde{c}_{-p_{1}}\right)^{\dagger} \tilde{c}_{-q_{1}} \tilde{c}_{-q_{2}}\right\rangle=-(-1)^{q_{2} / 2} \frac{2^{q_{2}}}{\left(q_{2}+1\right) !}\left[\delta_{q_{1},-1} \frac{2^{p_{1}}}{\left(p_{1}+1\right) !}(-1)^{\left(p_{1}+1\right) / 2}\right. \\
\left.+\delta_{p_{1},-1} \frac{2^{q_{1}}}{\left(q_{1}+1\right) !}(-1)^{\left(q_{1}+1\right) / 2}\right], \\
\left\langle\left(\tilde{c}_{-p_{1}}\right)^{\dagger}\left(\tilde{c}_{-p_{2}}\right)^{\dagger}\left(\mathcal{B}_{0}+\mathcal{B}_{0}^{\dagger}\right) \tilde{c}_{-q_{1}} \tilde{c}_{-q_{2}}\right\rangle \\
=-\left[\delta_{p_{2}, 0}(-1)^{q_{2} / 2} \frac{2^{q_{2}}}{\left(q_{2}+1\right) !}+\delta_{q_{2}, 0}(-1)^{p_{2} / 2} \frac{2^{p_{2}}}{\left(p_{2}+1\right) !}\right] \\
\times\left[\delta_{p_{1},-1} \frac{2^{q_{1}}}{\left(q_{1}+1\right) !}(-1)^{\left(q_{1}+1\right) / 2}+\delta_{q_{1},-1} \frac{2^{p_{1}}}{\left(p_{1}+1\right) !}(-1)^{\left(p_{1}+1\right) / 2}\right] .
\end{gathered}
$$

\section{Appendix E Details for ghost number one equation of motion}

In this appendix, we provide few intermediate steps for plugging the ansatz (4.2)

$$
\Psi=\sum_{n, p} f_{n, p} \widehat{\mathcal{L}}^{n} \tilde{c}_{p}|0\rangle+\sum_{n, p, q} f_{n, p, q} \widehat{\mathcal{B}} \widehat{\mathcal{L}}^{n} \tilde{c}_{p} \tilde{c}_{q}|0\rangle
$$


into the equation of motion $Q_{B} \Psi+\Psi * \Psi=0$. The action of the BRST charge $Q_{B}$ is quite simple, since it annihilates the vacuum, commutes with $\widehat{\mathcal{L}}^{n}$ and its anticommutator with $\widehat{\mathcal{B}}$ is $\widehat{\mathcal{L}}$. Least obvious is perhaps the action on the $\tilde{c}$ ghost $\left\{Q_{B}, \tilde{c}(\tilde{z})\right\}=\tilde{c} \tilde{\partial} \tilde{c}(\tilde{z})$, which takes the same form as in the $z$ coordinate. For the first term in the equation of motion, we find easily

$$
\begin{aligned}
Q_{B} \Psi= & \sum_{n, k, l}\left[\frac{k-l}{2} f_{n, k+l}+f_{n-1, k, l}\right] \widehat{\mathcal{L}}^{n} \tilde{c}_{k} \tilde{c}_{l}|0\rangle \\
& -\widehat{\mathcal{B}} \sum_{n, k, l, q}(k-l) f_{n, k+l, q} \widehat{\mathcal{L}}^{n} \tilde{c}_{k} \tilde{c}_{l} \tilde{c}_{q}|0\rangle
\end{aligned}
$$

For the second term $\Psi * \Psi$, we use results from Section 2.5. Denoting the two terms in (4.2) as $\Psi=\Psi^{(1)}+\Psi^{(2)}$, the second one containing the $\left(\mathcal{B}_{0}+\mathcal{B}_{0}^{\dagger}\right)$ factor, we find

$$
\begin{aligned}
& \Psi^{(1)} * \Psi^{(1)}=\sum_{N, n, m, k, l, p, q}(-1)^{k}\left(\frac{\pi}{4}\right)^{k+l} D_{n, m, l, k}^{N}\left(\begin{array}{c}
k+p-2 \\
k
\end{array}\right)\left(\begin{array}{c}
l+q-2 \\
l
\end{array}\right) \\
& \times f_{n, k+p} f_{m, l+q} \widehat{\mathcal{L}}^{N} \tilde{c}_{p} \tilde{c}_{q}|0\rangle \\
& \Psi^{(1)} * \Psi^{(2)}=\frac{\pi}{2} \sum_{N, n, m, k, l, p, q}(-1)^{k}\left(\frac{\pi}{4}\right)^{k+l} D_{n, m, k+l, 0}^{N}\left(\begin{array}{c}
k+p-2 \\
k
\end{array}\right)\left(\begin{array}{c}
l+q-2 \\
l
\end{array}\right) \\
& \times f_{n, 1} f_{m, p+k, q+l} \widehat{\mathcal{L}}^{N} \tilde{c}_{p} \tilde{c}_{q}|0\rangle-\sum_{N, n, m, k_{1}, k_{2}, l, p_{1}, p_{2}, q}\left(\frac{\pi}{4}\right)^{k_{1}+k_{2}+l} \\
& \times(-1)^{k_{1}} D_{n, m, k_{2}+l, k_{1}}^{N} f_{n, p_{1}+k_{1}} f_{m, p_{2}+k_{2}, q+l}\left(\begin{array}{c}
k_{1}+p_{1}-2 \\
k_{1}
\end{array}\right) \\
& \times\left(\begin{array}{c}
k_{2}+p_{2}-2 \\
k_{2}
\end{array}\right)\left(\begin{array}{c}
l+q-2 \\
l
\end{array}\right) \widehat{\mathcal{B}} \widehat{\mathcal{L}}^{N} \tilde{c}_{p_{1}} \tilde{c}_{p_{2}} \tilde{c}_{q}|0\rangle \\
& \Psi^{(2)} * \Psi^{(1)}=\frac{\pi}{2} \sum_{N, n, m, k, l, p, q}(-1)^{k}\left(-\frac{\pi}{4}\right)^{k+l} D_{n, m, 0, k+l}^{N}\left(\begin{array}{c}
k+p-2 \\
k
\end{array}\right)\left(\begin{array}{c}
l+q-2 \\
l
\end{array}\right) \\
& \times f_{n, p+k, q+l} f_{m, 1} \widehat{\mathcal{L}}^{N} \tilde{c}_{p} \tilde{c}_{q}|0\rangle+\sum_{N, n, m, k_{1}, k_{2}, l, p_{1}, p_{2}, q}\left(\frac{\pi}{4}\right)^{k_{1}+k_{2}+l} \\
& \times(-1)^{k_{1}+l} D_{n, m, k_{2}, k_{1}+l}^{N} f_{n, p_{1}+k_{1}, q+l} f_{m, p_{2}+k_{2}} \\
& \times\left(\begin{array}{c}
k_{1}+p_{1}-2 \\
k_{1}
\end{array}\right)\left(\begin{array}{c}
k_{2}+p_{2}-2 \\
k_{2}
\end{array}\right)\left(\begin{array}{c}
l+q-2 \\
l
\end{array}\right) \widehat{\mathcal{B}} \widehat{\mathcal{L}}^{N} \tilde{c}_{p_{1}} \tilde{c}_{q} \tilde{c}_{p_{2}}|0\rangle
\end{aligned}
$$




$$
\begin{aligned}
\Psi^{(2)} * \Psi^{(2)}= & \pi^{2} \sum_{N, n, m, q_{1}, q_{2}, l_{1}, l_{2}}(-1)^{l_{1}}\left(\frac{\pi}{4}\right)^{l_{1}+l_{2}} D_{n, m, l_{2}, l_{1}}^{N}\left(\begin{array}{c}
l_{1}+q_{1}-2 \\
l_{1}
\end{array}\right) \\
& \times\left(\begin{array}{c}
l_{2}+q_{2}-2 \\
l_{2}
\end{array}\right) f_{n, 1, q_{1}} f_{m, 1, q_{2}} \widehat{\mathcal{L}}^{N} \tilde{c}_{q_{1}} \tilde{c}_{q_{2}}|0\rangle \\
& -\frac{\pi}{2} \sum_{N, n, m, p_{1}, p_{2}, q_{1}, q_{2}, k_{1}, k_{2}, l_{1}, l_{2}}\left(\frac{\pi}{4}\right)^{k_{1}+l_{1}+k_{2}+l_{2}}(-1)^{k_{1}+l_{1}} \\
& \times D_{n, m, k_{2}+l_{2}, k_{1}+l_{1}}^{N} f_{n, p_{1}+k_{1}, q_{1}+l_{1}} f_{m, p_{2}+k_{2}, q_{2}+l_{2}} \\
& \times\left(\begin{array}{c}
k_{1}+p_{1}-2 \\
k_{1}
\end{array}\right)\left(\begin{array}{c}
l_{1}+q_{1}-2 \\
l_{1}
\end{array}\right)\left(\begin{array}{c}
k_{2}+p_{2}-2 \\
k_{2}
\end{array}\right)\left(\begin{array}{c}
l_{2}+q_{2}-2 \\
l_{2}
\end{array}\right) \\
& \times \widehat{\mathcal{B}} B_{1} \widehat{\mathcal{L}}^{N} \tilde{c}_{p_{1}} \tilde{c}_{q_{1}} \tilde{c}_{p_{2}} \tilde{c}_{q_{2}}|0\rangle,
\end{aligned}
$$

where

$$
\begin{aligned}
D_{n, m, k, l}^{N} & =\frac{n ! m !}{N !}(-2)^{n+m-N} \oint \frac{d r}{2 \pi i} \oint \frac{d s}{2 \pi i} \frac{(r+s-3)^{N}}{(r-2)^{n+1}(s-2)^{n+1}}(r-1)^{k}(s-1)^{l} \\
& =\frac{n ! m !}{N !}(-2)^{n+m-N} \sum_{j=0}^{N}\left(\begin{array}{c}
N \\
j
\end{array}\right)\left(\begin{array}{c}
k \\
n-j
\end{array}\right)\left(\begin{array}{c}
N-j+l \\
m
\end{array}\right) .
\end{aligned}
$$

Although the 12-fold sum with up to seven binomial factors looks prohibitively complicated, it is actually quite easy to plug the expressions to the computer. Imposing twist symmetry, i.e., $f_{n, p}=0$ for $p$ even and $f_{n, p, q}=0$ for $p+q$ even, we find

$$
\begin{aligned}
0= & f_{0,1}+\pi\left[-\frac{1}{2} f_{0,1}^{2}+f_{0,1}\left(f_{1,1}+2 f_{0,1,0}\right)\right] \\
0= & f_{1,1}+2 f_{0,1,0}+\pi\left[\frac{1}{4} f_{0,1}^{2}-\frac{3}{2} f_{0,1} f_{1,1}-f_{0,1} f_{0,1,0}+f_{1,1}^{2}+2 f_{1,1} f_{0,1,0}\right. \\
& \left.+2 f_{0,1}\left(f_{2,1}+f_{1,1,0}\right)\right] \\
0= & f_{2,1}+2 f_{1,1,0}+\pi\left[-\frac{1}{16} f_{0,1}^{2}+\frac{5}{8} f_{0,1} f_{1,1}-f_{1,1}^{2}-2 f_{0,1} f_{2,1}+3 f_{1,1} f_{2,1}\right. \\
& +f_{0,1}\left(3 f_{3,1}+2 f_{2,1,0}\right)+\frac{1}{4} f_{0,1} f_{0,1,0}-f_{1,1} f_{0,1,0}+2 f_{2,1} f_{0,1,0}-f_{0,1} f_{1,1,0} \\
& \left.+2 f_{1,1} f_{1,1,0}\right]
\end{aligned}
$$




$$
\begin{aligned}
0= & f_{0,-1}+\pi\left[\frac{1}{2} f_{0,1} f_{0,-1}+f_{0,1}\left(-f_{1,-1}+2 f_{0,0,-1}\right)\right]+\pi^{3}\left[\frac{1}{32} f_{0,1}^{2}-\frac{3}{16} f_{0,1} f_{1,1}\right. \\
& +\frac{1}{4} f_{1,1}^{2}+\frac{1}{4} f_{0,1} f_{2,1}-\frac{1}{2} f_{1,1} f_{2,1}+\frac{1}{8} f_{0,1} f_{0,1,0}-\frac{1}{2} f_{1,1} f_{0,1,0}+f_{2,1} f_{0,1,0} \\
& \left.-f_{0,1,0}^{2}+2 f_{0,1,0} f_{1,1,0}\right] \\
0= & 3 f_{0,-1}+\pi\left[-\frac{3}{2} f_{0,-1} f_{0,1}+3 f_{0,-1} f_{1,1}+2 f_{0,1} f_{0,1,-2}\right]+\pi^{3}\left[-\frac{1}{32} f_{0,1}^{2}\right. \\
& +\frac{3}{16} f_{0,1} f_{1,1}-\frac{3}{4} f_{0,1} f_{2,1}+\frac{3}{2} f_{0,1} f_{3,1}+\frac{3}{8} f_{0,1} f_{0,1,0}-\frac{3}{2} f_{1,1} f_{0,1,0} \\
& \left.+3 f_{2,1} f_{0,1,0}\right] \ldots
\end{aligned}
$$

These equations are equations for the coefficients of $\tilde{c}_{1} \tilde{c}_{0}|0\rangle,\left(\mathcal{L}_{0}+\mathcal{L}_{0}^{\dagger}\right) \tilde{c}_{1} \tilde{c}_{0}|0\rangle$, $\left(\mathcal{L}_{0}+\mathcal{L}_{0}^{\dagger}\right)^{2} \tilde{c}_{1} \tilde{c}_{0}|0\rangle, \tilde{c}_{0} \tilde{c}_{-1}|0\rangle, \tilde{c}_{1} \tilde{c}_{-2}|0\rangle, \ldots$ in the equation of motion $Q_{B} \Psi+$ $\Psi * \Psi=0$. It is interesting to see that imposing the $\mathcal{B}_{0}$ gauge condition

$$
f_{n, p, 0}+\frac{n+1}{2} f_{n+1, p}=0
$$

eliminates all the terms in the round brackets, and therefore, the equations become exactly solvable one after the other. We have proved this general pattern in Section 4. For example the first equation implies

$$
f_{0,1}=\frac{2}{\pi}, \quad \text { or } \quad f_{0,1}=0 .
$$

In the first case $f_{0,1}=\frac{2}{\pi}$, we readily find

$$
f_{1,1}=\frac{1}{2 \pi}, \quad f_{2,1}=\frac{1}{24 \pi}, \quad f_{0,-1}=\frac{\pi}{48}, \quad f_{3,1}=-\frac{4}{3 \pi^{2}} f_{0,1,-2}
$$

and so on. Continuing up to level 12 , i.e., finding coefficients like $f_{12,1}$, it is easy to guess the complete form

$$
\begin{aligned}
f_{n,-p} & =\frac{\pi^{p}}{2^{n+2 p+1} n !}(-1)^{n} B_{n+p+1}, \quad p \text { odd }, \\
f_{n,-p,-q} & =\frac{\pi^{p+q}}{2^{n+2(p+q)+3} n !}(-1)^{n+q} B_{n+p+q+2}, \quad p+q \text { odd },
\end{aligned}
$$

and hence (4.9) follows. The only proof that our guess is a true solution is given in Section 4.1 using the wedge state representation. From the mathematical point of view, it would be interesting to find a direct proof using the form (4.9), since this would presumably lead to an infinite set of EulerRamanujan type of identities for Bernoulli numbers. 


\section{E.1 Pure gauge solutions}

In the second case $f_{0,1}=0$, we find $f_{1,1}=\beta$ to be a free parameter which determines

$$
f_{2,1}=-\frac{\pi}{2} \beta^{2}, \quad f_{0,-1}=-\frac{\pi^{3}}{4} \beta^{2} .
$$

Going one step further, we would find

$$
\begin{gathered}
f_{3,1}=\frac{\pi}{8} \beta^{2}+\frac{\pi^{2}}{4} \beta^{3}, \quad f_{1,-1}=\frac{3 \pi^{3}}{16} \beta^{2}+\frac{3 \pi^{4}}{8} \beta^{3}, \\
f_{0,1,-2}=-\frac{3 \pi^{3}}{32} \beta^{2}-\frac{3 \pi^{4}}{16} \beta^{3} .
\end{gathered}
$$

This solution clearly corresponds to a pure gauge. One particular value of $\beta$ deserves perhaps a special attention. For $\beta=-\frac{1}{2 \pi}$, we found that all the terms $f_{3,1}, f_{1,-1}, f_{0,1,-2}$ vanish, and it seems that the solution shares the symmetry (4.43) with the tachyon solution. The other low level coefficients for this value of $\beta$ are given by

$$
\begin{gathered}
f_{2,1}=-\frac{1}{8 \pi}, \quad f_{0,-1}=-\frac{\pi}{16}, \\
f_{4,1}=\frac{1}{384 \pi}, \quad f_{2,-1}=-f_{1,1,-2}=\frac{\pi}{128}, \quad f_{0,-3}=\frac{\pi^{3}}{256} .
\end{gathered}
$$

\section{Appendix F Coefficients of the tachyon condensate in the Virasoro basis}

Complete table of the exact coefficients up to level 4 is

\begin{tabular}{ll}
\hline$c_{1}|0\rangle$ & 0.55346558 \\
$c_{-1}|0\rangle$ & 0.45661043 \\
$L_{-2} c_{1}|0\rangle$ & 0.13764616 \\
$b_{-2} c_{0} c_{1}|0\rangle$ & -0.14421001 \\
$L_{-4} c_{1}|0\rangle$ & -0.030277583 \\
$L_{-2} L_{-2} c_{1}|0\rangle$ & 0.0045805832 \\
$c_{-3}|0\rangle$ & -0.16494614 \\
$b_{-3} c_{-1} c_{1}|0\rangle$ & 0.16039444 \\
$b_{-2} c_{-2} c_{1}|0\rangle$ & 0.17942652 \\
$L_{-2} c_{-1}|0\rangle$ & 0.022748278 \\
$L_{-3} c_{0}|0\rangle$ & 0 \\
$b_{-2} c_{-1} c_{0}|0\rangle$ & 0.020943544 \\
$b_{-4} c_{0} c_{1}|0\rangle$ & 0.088982260 \\
$L_{-2} b_{-2} c_{0} c_{1}|0\rangle$ & -0.0084696519 \\
\hline
\end{tabular}


Let us also list the coefficients in the matter sector up to level 10

\begin{tabular}{ll}
\hline$c_{1}|0\rangle$ & 0.55346558 \\
$L_{-2} c_{1}|0\rangle$ & 0.13764616 \\
$L_{-4} c_{1}|0\rangle$ & -0.030277583 \\
$L_{-2} L_{-2} c_{1}|0\rangle$ & 0.0045805832 \\
$L_{-6} c_{1}|0\rangle$ & 0.01245732489 \\
$L_{-4} L_{-2} c_{1}|0\rangle$ & -0.0015475008 \\
$L_{-2} L_{-2} L_{-2} c_{1}|0\rangle$ & -0.00015818471 \\
$L_{-8} c_{1}|0\rangle$ & -0.00694735218 \\
$L_{-6} L_{-2} c_{1}|0\rangle$ & 0.000722255152 \\
$L_{-4} L_{-4} c_{1}|0\rangle$ & 0.0001290340047 \\
$L_{-4} L_{-2} L_{-2} c_{1}|0\rangle$ & 0.000085720253 \\
$L_{-2} L_{-2} L_{-2} L_{-2} c_{1}|0\rangle$ & $2.552937710^{-6}$ \\
$L_{-10} c_{1}|0\rangle$ & 0.004375158716 \\
$L_{-8} L_{-2} c_{1}|0\rangle$ & -0.000396885628 \\
$L_{-6} L_{-4} c_{1}|0\rangle$ & -0.000120886555 \\
$L_{-6} L_{-2} L_{-2} c_{1}|0\rangle$ & -0.000039274125 \\
$L_{-4} L_{-4} L_{-2} c_{1}|0\rangle$ & -0.000015086291 \\
$L_{-4} L_{-2} L_{-2} L_{-2} c_{1}|0\rangle$ & $-2.286398910^{-6}$ \\
$L_{-2} L_{-2} L_{-2} L_{-2} L_{-2} c_{1}|0\rangle$ & $4.067479810^{-8}$ \\
\hline
\end{tabular}

It is worth noticing that the coefficients of the states $L_{-2}^{n} c_{1}|0\rangle$ decay quite rapidly at a similar rate as in the Siegel gauge. This can be contrasted with identity based solution, where the decay is much slower, leading eventually to the divergence of the energy.

\section{References}

[1] E. Witten, Noncommutative geometry and string field theory, Nucl. Phys. B 268 (1986), 253.

[2] W. Taylor and B. Zwiebach, D-branes, tachyons, and string field theory, arXiv:hep-th/0311017.

[3] A. Sen, Tachyon dynamics in open string theory, Int. J. Mod. Phys. A 20 (2005), 5513; arXiv:hep-th/0410103.

[4] L. Rastelli, A. Sen and B. Zwiebach, String field theory around the tachyon vacuum, Adv. Theor. Math. Phys. 5 (2002), 353; arXiv:hepth/0012251.

[5] A. Sen and B. Zwiebach, Tachyon condensation in string field theory, JHEP 0003 (2000), 002; arXiv:hep-th/9912249. 
[6] V.A. Kostelecký and S. Samuel, On a nonperturbative vacuum for the open Bosonic string, Nucl. Phys. B 336 (1990), 263.

[7] K. Bardakci, Dual models and spontaneous symmetry breaking, Nucl. Phys. B 68 (1974), 331.

[8] K. Bardakci and M.B. Halpern, Explicit spontaneous breakdown in a dual model, Phys. Rev. D 10 (1974), 4230.

[9] K. Bardakci and M.B. Halpern, Explicit spontaneous breakdown in a dual model. 2. N point functions, Nucl. Phys. B 96 (1975), 285.

[10] K. Bardakci, Spontaneous symmetry breakdown in the standard dual string model, Nucl. Phys. B 133 (1978), 297.

[11] A. Sen, Descent relations among bosonic D-branes, Int. J. Mod. Phys. A 14 (1999), 4061; arXiv:hep-th/9902105.

[12] A. Sen, Universality of the tachyon potential, JHEP 9912 (1999), 027; arXiv:hep-th/9911116.

[13] A.A. Gerasimov and S.L. Shatashvili, On exact tachyon potential in open string field theory, JHEP 0010 (2000), 034; arXiv:hep-th/ 0009103.

[14] D. Kutasov, M. Marino and G.W. Moore, Some exact results on tachyon condensation in string field theory, JHEP 0010 (2000), 045; arXiv:hepth/0009148.

[15] D. Ghoshal and A. Sen, Normalisation of the background independent open string field theory action, JHEP 0011 (2000), 021; arXiv:hepth/0009191.

[16] L. Rastelli, A. Sen and B. Zwiebach, Boundary CFT construction of D-branes in vacuum string field theory, JHEP 0111, (2001), 045; arXiv:hep-th/0105168.

[17] K. Okuyama, Ratio of tensions from vacuum string field theory, JHEP 0203 (2002), 050; arXiv:hep-th/0201136.

[18] Y. Okawa, Open string states and D-brane tension from vacuum string field theory, JHEP 0207 (2002), 003; arXiv:hep-th/0204012.

[19] N. Moeller and W. Taylor, Level truncation and the tachyon in open bosonic string field theory, Nucl. Phys. B 583 (2000), 105; arXiv:hepth/0002237.

[20] D. Gaiotto and L. Rastelli, Experimental string field theory, JHEP 0308 (2003), 048; arXiv:hep-th/0211012.

[21] J.A. Harvey and P. Kraus, D-branes as unstable lumps in bosonic open string field theory, JHEP 0004 (2000), 012; arXiv:hep-th/0002117.

[22] R. de Mello Koch, A. Jevicki, M. Mihailescu and R. Tatar, Lumps and p-branes in open string field theory, Phys. Lett. B 482 (2000), 249; arXiv:hep-th/0003031. 
[23] N. Moeller, A. Sen and B. Zwiebach, D-branes as tachyon lumps in string field theory, JHEP 0008, (2000), 039; arXiv:hep-th/0005036.

[24] I. Ellwood and W. Taylor, Open string field theory without open strings, Phys. Lett. B 512, (2001), 181; arXiv:hep-th/0103085.

[25] I. Ellwood, B. Feng, Y.H. He and N. Moeller, The identity string field and the tachyon vacuum, JHEP 0107 (2001), 016; arXiv:hepth/0105024.

[26] S. Giusto and C. Imbimbo, Physical states at the tachyonic vacuum of open string field theory, Nucl. Phys. B 677, (2004), 52; arXiv:hepth/0309164.

[27] K. Ohmori, A review on tachyon condensation in open string field theories, arXiv:hep-th/0102085.

[28] P.J. De Smet, Tachyon condensation: Calculations in string field theory, arXiv:hep-th/0109182.

[29] I.Y. Arefeva, D.M. Belov, A.A. Giryavets, A.S. Koshelev and P.B. Medvedev, Noncommutative field theories and (super)string field theories, arXiv:hep-th/0111208.

[30] L. Bonora, C. Maccaferri, D. Mamone and M. Salizzoni, Topics in string field theory, arXiv:hep-th/0304270.

[31] H. Hata and S. Shinohara, BRST invariance of the non-perturbative vacuum in bosonic open string field theory, JHEP 0009 (2000), 035; arXiv:hep-th/0009105.

[32] B. Zwiebach, Trimming the tachyon string field with $S U(1,1)$, arXiv:hep-th/0010190.

[33] M. Schnabl, Constraints on the tachyon condensate from anomalous symmetries, Phys. Lett. B 504 (2001), 61; arXiv:hep-th/0011238.

[34] D. Gaiotto, L. Rastelli, A. Sen and B. Zwiebach, Patterns in open string field theory solutions, JHEP 0203 (2002), 003; arXiv:hep-th/0201159.

[35] M. Schnabl, String field theory at large B-field and noncommutative geometry, JHEP 0011, (2000), 031; arXiv:hep-th/0010034.

[36] J. Klusoň, Exact solutions of open bosonic string field theory, JHEP 0204 (2002), 043; arXiv:hep-th/0202045.

[37] I. Kishimoto and K. Ohmori, CFT description of identity string field: Toward derivation of the VSFT action, JHEP 0205 (2002), 036; arXiv:hep-th/0112169.

[38] T. Takahashi and S. Tanimoto, Marginal and scalar solutions in cubic open string field theory, JHEP 0203 (2002), 033; arXiv:hep-th/0202133.

[39] V.A. Kostelecký and R. Potting, Analytical construction of a nonperturbative vacuum for the open bosonic string, Phys. Rev. D 63 (2001), 046007; arXiv:hep-th/0008252. 
[40] Y. Okawa, Solving Witten's string field theory using the butterfly state, Phys. Rev. D 69 (2004), 086001; arXiv:hep-th/0311115.

[41] H.t. Yang, Solving Witten's SFT by insertion of operators on projectors, JHEP 0409 (2004), 002; arXiv:hep-th/0406023.

[42] I. Kishimoto and T. Takahashi, Open string field theory around universal solutions, Prog. Theor. Phys. 108 (2002), 591; arXiv:hepth/0205275.

[43] T. Takahashi, Tachyon condensation and universal solutions in string field theory, Nucl. Phys. B 670 (2003), 161; arXiv:hep-th/0302182.

[44] T. Takahashi and S. Zeze, Gauge fixing and scattering amplitudes in string field theory around universal solutions, Prog. Theor. Phys. 110 (2003), 159; arXiv:hep-th/0304261.

[45] I. Bars, Map of Witten's* to Moyal's*, Phys. Lett. B 517 (2001), 436; arXiv:hep-th/0106157.

[46] L. Rastelli, A. Sen and B. Zwiebach, Star algebra spectroscopy, JHEP 0203 (2002), 029; arXiv:hep-th/0111281.

[47] M.R. Douglas, H. Liu, G.W. Moore and B. Zwiebach, Open string star as a continuous Moyal product, JHEP 0204 (2002), 022; arXiv:hepth/0202087.

[48] I. Bars and Y. Matsuo, Computing in string field theory using the Moyal star product, Phys. Rev. D 66 (2002), 066003; arXiv:hep-th/0204260.

[49] D.M. Belov and C. Lovelace, Star products made easy, Phys. Rev. D 68 (2003), 066003; arXiv:hep-th/0304158.

[50] S.B. Giddings, The Veneziano amplitude from interacting string field theory, Nucl. Phys. B 278 (1986), 242.

[51] J.H. Sloan, The scattering amplitude for four off-shell tachyons from functional integrals, Nucl. Phys. B 302 (1988), 349.

[52] S. Samuel, Covariant off-shell string amplitudes, Nucl. Phys. B 308 (1988), 285.

[53] W. Taylor, Perturbative diagrams in string field theory, arXiv:hepth/0207132.

[54] L. Rastelli and B. Zwiebach, Tachyon potentials, star products and universality, JHEP 0109 (2001), 038; arXiv:hep-th/0006240.

[55] M. Schnabl, Wedge states in string field theory, JHEP 0301 (2003), 004; arXiv:hep-th/0201095.

[56] C.R. Preitschopf, C.B. Thorn and S.A. Yost, Superstring field theory, Nucl. Phys. B 337 (1990), 363.

[57] A. LeClair, M.E. Peskin and C.R. Preitschopf, String field theory on the conformal plane. 1. Kinematical principles, Nucl. Phys. B 317 (1989), 411. 
[58] A. LeClair, M.E. Peskin and C.R. Preitschopf, String field theory on the conformal plane. 2. Generalized gluing, Nucl. Phys. B 317 (1989), 464.

[59] B. Zwiebach, Closed string field theory: quantum action and the $B-V$ master equation, Nucl. Phys. B 390 (1993), 33; arXiv:hep-th/9206084.

[60] M. Schnabl, Remarks on surface states and derivations in string field theory, Progress in String, Field and Particle Theory, Cargese, Corsica, France, 25 Jun - 11 Jul 2002, Kluwer Academic Publishers.

[61] L. Rastelli, A. Sen and B. Zwiebach, Classical solutions in string field theory around the tachyon vacuum, Adv. Theor. Math. Phys. 5 (2002), 393; arXiv:hep-th/0102112.

[62] G.H. Hardy, Divergent series, 2nd edition, Chelsea Publishing Company, New York, 1991.

[63] G.T. Horowitz and S.P. Martin, Conformal field theory and the symmetries of string field theory, Nucl. Phys. B 296 (1988), 220.

[64] G.T. Horowitz and A. Strominger, Translations as inner derivations and associativity anomalies in open string field theory, Phys. Lett. B 185 (1987), 45.

[65] W. Siegel and B. Zwiebach, Gauge string fields, Nucl. Phys. B 263, (1986), 105.

[66] W. Taylor, A perturbative analysis of tachyon condensation, JHEP 0303 (2003), 029; arXiv:hep-th/0208149.

[67] D. Gaiotto, L. Rastelli, A. Sen and B. Zwiebach, Star algebra projectors, JHEP 0204 (2002), 060; arXiv:hep-th/0202151.

[68] D. Gaiotto, L. Rastelli, A. Sen and B. Zwiebach, Ghost structure and closed strings in vacuum string field theory, Adv. Theor. Math. Phys. 6 (2003), 403; arXiv:hep-th/0111129.

[69] M. Schnabl, Anomalous reparametrizations and butterfly states in string field theory, Nucl. Phys. B 649 (2003), 101; arXiv:hep-th/0202139.

[70] A. Boyarsky and O. Ruchayskiy, Integrability in SFT and new representation of KP tau-function, JHEP 0303 (2003), 027; arXiv:hepth/0211010.

[71] L. Bonora and A.S. Sorin, Integrable structures in string field theory, Phys. Lett. B 553 (2003), 317; arXiv:hep-th/0211283.

[72] E.W. Weisstein. Bernoulli number. From Mathworld-a wolfram web resource. http://mathworld.wolfram.com/BernoulliNumber.html

[73] E.T. Whittaker and G.N. Watson, A course of modern analysis, 4th edition, Cambridge University Press, 1952.

[74] H.M. Edwards, Riemann's zeta function, Academic Press, 1974. 
